\title{
Model and Stability Analysis of a Flexible Bladed Rotor
}

\author{
N. Lesaffre, J.-J. Sinou, and F. Thouverez \\ Equipe Dynamique des Systèmes, UMR CNRS 5513, Laboratoire de Tribologie et Dynamique des Systèmes, \\ École Centrale de Lyon, 36 venue Guy de Collongue, 69134 Ecully Cedex, France
}

Received 8 April 2005; Accepted 7 November 2005

This paper presents a fully bladed flexible rotor and outlines the associated stability analysis. From an energetic approach based on the complete energies and potentials for Euler-Bernoulli beams, a system of equations is derived, in the rotational frame, for the rotor. This later one is made of a hollow shaft modelled by an Euler-Bernoulli beam supported by a set of bearings. It is connected to a rigid disk having a rotational inertia. A full set of flexible blades is also modelled by Euler-Bernoulli beams clamped in the disk. The flexural vibrations of the blades as well as those of the shaft are considered. The evolution of the eigenvalues of this rotor, in the corotational frame, is studied. A stability detection method, bringing coalescence and loci separation phenomena to the fore, in case of an asymmetric rotor, is undertaken in order to determine a parametric domain where turbomachinery cannot encounter damage. Finally, extensive parametric studies including the length and the stagger angle of the blades as well as their flexibility are presented in order to obtain robust criteria for stable and unstable areas prediction.

Copyright $\odot 2006$ N. Lesaffre et al. This is an open access article distributed under the Creative Commons Attribution License, which permits unrestricted use, distribution, and reproduction in any medium, provided the original work is properly cited.

\section{INTRODUCTION}

Rotor dynamics is a very particular and rich field of mechanics, where plenty of phenomena [1-4] can be responsible for the instabilities of rotating structures. A common particular interest of the rotating machinery industry is to understand correctly the vibration phenomena and to predict the dynamic behaviour of flexible bladed rotors. Effectively, a sufficient knowledge of the vibration phenomena is essential for considering adequate means to reduce or eliminate vibrations and for designing rotating machinery. Thus, the instability of propellers due to the interaction between the dynamics of the blades and that of the engine suspension is a well-known phenomenon and has attracted the interest of Crandall and Dugundji [5]. This kind of instability may also occur in bladed rotors, like those present in turbomachinery [6].

The master of such structure is fundamental for industry due to the fact that instabilities of bladed rotors can cause severe damage. In order to avoid these problems, engineers need therefore to find suitable devices to eliminate instabilities. Unfortunately, this kind of study is quite complex and must be performed using numerical models $[7,8]$. However, to obtain a closed-form solution suitable for a stability analysis, it is possible to resort to simplified models [9-11].
In this paper, a simplified model of a fully bladed flexible rotor is firstly presented. In a second part, a stability analysis and parametric studies are investigated in order to detect the stable and unstable regions of this structure in case of asymmetries. The paper closes with a discussion of some topics of future interest and some conclusions.

\section{MODEL FORMULATION}

The rotor considered in this study has been developed on an energetic approach based on the same kind of approximation as the one described by Sinha [9]. It consists of a shaft modelled by an Euler-Bernoulli beam, connected to a rigid disk modelled by a concentrated mass with rotational inertia. Several Euler-Bernoulli beams are clamped in the disk and model the blades. The shaft is set on bearings at multiple locations, as indicated in Figure 1.

Two degrees of freedom are considered for the shaft: two orthogonal translations $u(z, t)$ and $v(z, t)$ in the disk's plane, and one degree of freedom for each blade defining its deflection $\eta(s, t)$, as illustrated in Figure 2.

A Rayleigh-Ritz approximation is used to express the degrees of freedom of these different parts. Thus, they are expressed by a sum of shape functions multiplied by 


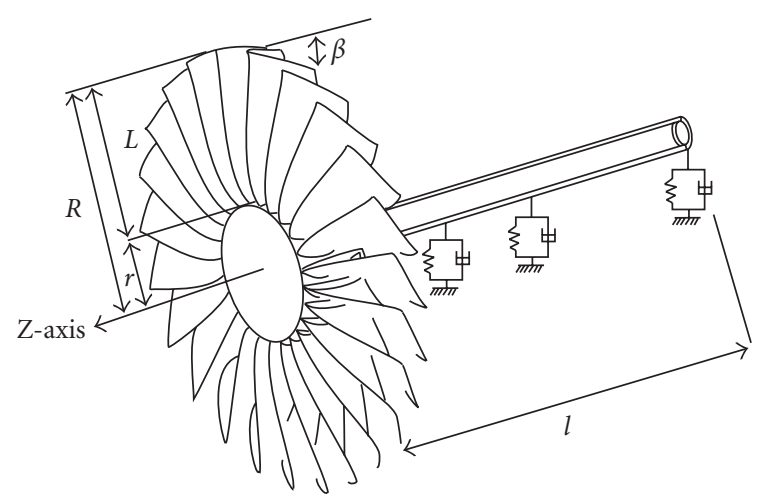

Figure 1: Model of flexible bladed rotor [9].

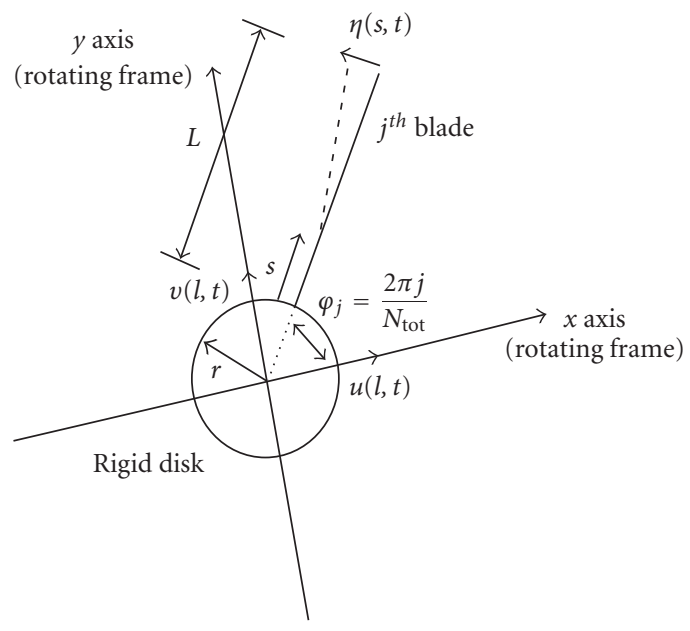

FIgUre 2: Front view of the bladed disk.

time-dependent coefficients:

$$
\begin{gathered}
u(z, t)=U_{o}(t)+\sum_{m=1}^{m_{\text {tot }}} U_{m}(t) W_{m}(z), \\
v(z, t)=V_{o}(t)+\sum_{m=1}^{m_{\text {tot }}} V_{m}(t) W_{m}(z), \\
\eta(s, t)=\sum_{n=1}^{n_{\text {tot }}}\left(X_{n}(t)\right)_{j}\left(Y_{n}(s)\right)_{j},
\end{gathered}
$$

where $z$ is the axis of the shaft and $s$ the axis along the blade. In these expressions, $U_{o}(t)$ and $V_{o}(t)$ are rigid body translations of the shaft. $m_{\text {tot }}$ and $n_{\text {tot }}$ are the number of modes considered to express its motion and the flexure of the $j$ th blade, respectively. In this Rayleigh-Ritz approach, the shape functions have only to verify the geometric boundary conditions of the problem, so the shape functions of the blades must verify

$$
Y_{n}(0)=0, \quad Y_{n}^{\prime}(0)=0,
$$

where prime denotes differentiation versus space coordinates, because they are clamped in the rigid disk. Thus, the chosen expression for the shape function of the blade deflection is

$$
Y_{n}(s)=a_{n} s+\sin \left(\beta_{n} s\right),
$$

with $\beta_{n}=(2 n-1) \pi /(2 L)$, and $a_{n}=-\beta_{n}$.

Concerning the shaft, since it is supported by bearings, its shape function has no geometric boundary conditions to verify. It can thus be defined by

$$
W_{m}(z)=1-\cos \left(\alpha_{m} z\right),
$$

with $\alpha_{m}=(2 m-1) \pi /(2 l)$. However, it could be noticed that these functions verify

$$
W_{m}(0)=0, \quad W_{m}^{\prime}(0)=0,
$$

thus no motion is permitted at the end of the shaft (i.e., at $z=0)$.

An energetic method is used to develop this model, thus potentials have to be defined for the shaft and for the blades as well. The model has been fully developed in the rotating frame in order to avoid having time-dependent terms, resulting from the periodicity of the rotating structure, in the analytical formulation. Thus, the kinetic energy $T_{\text {blade }}$ of the $j$ th blade, located at an angle $\varphi_{j}=2 \pi j / N_{\text {tot }}$ in this frame, as indicated in Figure 2 and where $N_{\text {tot }}$ is the total number of blades, can be fully expressed by the following relation:

$$
T_{\text {blade }}=\int_{0}^{L} \frac{1}{2} \rho_{b} S_{b} \vec{V}_{S / R_{0}}^{2}(G) d s+\int_{0}^{L} \frac{1}{2} \vec{\Omega}_{S / R_{0}}^{T} \mathbf{I} \vec{\Omega}_{S / R_{0}} d s,
$$

where $\rho_{b}$ and $S_{b}$ are the density and the area, respectively, of a blade cross-section. $\vec{V}_{S / R_{0}}(G), \vec{\Omega}_{S / R_{0}}$, and I are the speed and the rotation of the center of mass of a blade cross-section in relation to the fixed frame and its inertia matrix, defined in its inertial frame. It should be noticed that the complete expression of the kinetic energy should be considered because in certain extreme geometric conditions, the approximated kinetic energy can lead to unrealistic instabilities of the rotor. 


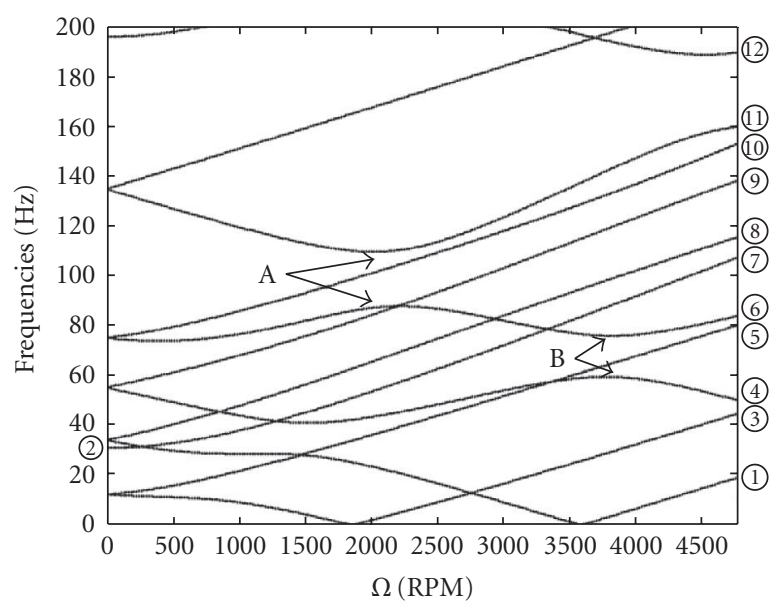

FIGURE 3: Campbell diagram for a twenty-eight-bladed rotor with blades of one meter in length.

The potential energy $v_{\text {int thade }}$ associated with the elastic deformation of the blade, a function of dissipation $F_{d_{b l a d e}}$ associated with the internal damping of the $j$ th blade, and a prestress potential taking into account the effect of the rotational inertia are formulated:

$$
v_{g_{\text {blade }}}=\frac{1}{2} \int_{0}^{L} \rho_{b} S_{b} \Omega^{2}\left[\frac{R^{2}-(s+r)^{2}}{2}\right] \eta^{\prime 2}(s, t) d s .
$$

In the same way, the kinetic energy $T_{\text {shaft }}$, the potential energy $v_{\text {int }_{\text {shaft }}}$ associated with the elastic deformation of the shaft, a function of dissipation $F_{d_{\text {shaft }}}$ associated with the internal damping of the shaft, as well as a potential $\nu_{\text {bearings }}$ and a function of dissipation $F_{d_{\text {bearings }}}$ associated with the bearings are defined.

$$
\mathbf{X}_{\text {rotor }}=\left[\begin{array}{llllllllllllll}
U_{0} & V_{0} & U_{1} & V_{1} & \cdots & U_{m_{\mathrm{tot}}} & V_{m_{\mathrm{tot}}} & X_{11} & X_{21} & \cdots & X_{n_{\mathrm{tot}} 1} & \cdots & X_{1 N_{\mathrm{tot}}} & X_{n_{\mathrm{tot}} N_{\mathrm{tot}}}
\end{array}\right]^{T} .
$$

Then, Lagrange's equations are used in order to obtain the system of equations of the dynamic behaviour of the full flexible bladed rotor. This system of equations can be written under the following form:

$$
\mathbf{M}_{\text {rotor }} \ddot{\mathbf{X}}_{\text {rotor }}+\widetilde{\mathbf{C}}_{\text {rotor }} \dot{\mathbf{X}}_{\text {rotor }}+\widetilde{\mathbf{K}}_{\text {rotor }} \mathbf{X}_{\text {rotor }}=\mathbf{0} \text {, }
$$

where $\mathbf{M}_{\text {rotor }}, \widetilde{\mathbf{C}}_{\text {rotor }}$, and $\widetilde{\mathbf{K}}_{\text {rotor }}$ are the mass matrix, the generalized damping matrix, and the generalized stiffness matrix, respectively. $\mathbf{X}_{\text {rotor }}$ defines the generalized degree of freedom vector of the rotor. It contains $2 m_{\text {tot }}+2+n_{\text {tot }} N_{\text {tot }}$ elements and has the following expression:
All expressions of the energies and potentials are given in Appendix A as well as those of the mass, damping, and stiffness matrices, in Appendix B.

\section{STABILITY ANALYSIS}

One of the most important objectives for turbomachinery designers is to define dangerous operating speed ranges and the associated stable and unstable regions of bladed rotor vibrations.

The stability of the flexible bladed rotor is investigated by determining the solution $\lambda=a+i b$ of the characteristic equation

$$
\operatorname{det}\left(\lambda^{2} \mathbf{M}_{\text {rotor }}+\lambda \widetilde{\mathbf{C}}_{\text {rotor }}+\widetilde{\mathbf{K}}_{\text {rotor }}\right)=0 .
$$

It becomes unstable if any one or more of the eigenvalues' real parts $a$ is positive. The imaginary parts define the frequencies of the system.

In this following study, we consider a shaft supported by three isotropic bearings located at $z=0 \mathrm{~cm}, z=300 \mathrm{~cm}$, and $z=325 \mathrm{~cm}$. The radial stiffness and viscous damping coefficients for these bearings are $k_{\text {bearing }}=2 \cdot 10^{7} \mathrm{~N} \cdot \mathrm{m}^{-1}$ and $D_{\text {bearing }}=2 \cdot 10^{3}$ N.s.m ${ }^{-1}$.

Figure 3 illustrates the evolution of the eigenfrequencies of a flexible twenty-eight-bladed rotor of one meter in length, plotted in a Campbell diagram. Figure 4 shows the evolution of the eigenvalues in the complex plane. The evolutions of the shaft's eigenfrequencies, shown in Figure 3, are mainly due to the gyroscopic effects. In the rotating frame, the downward 


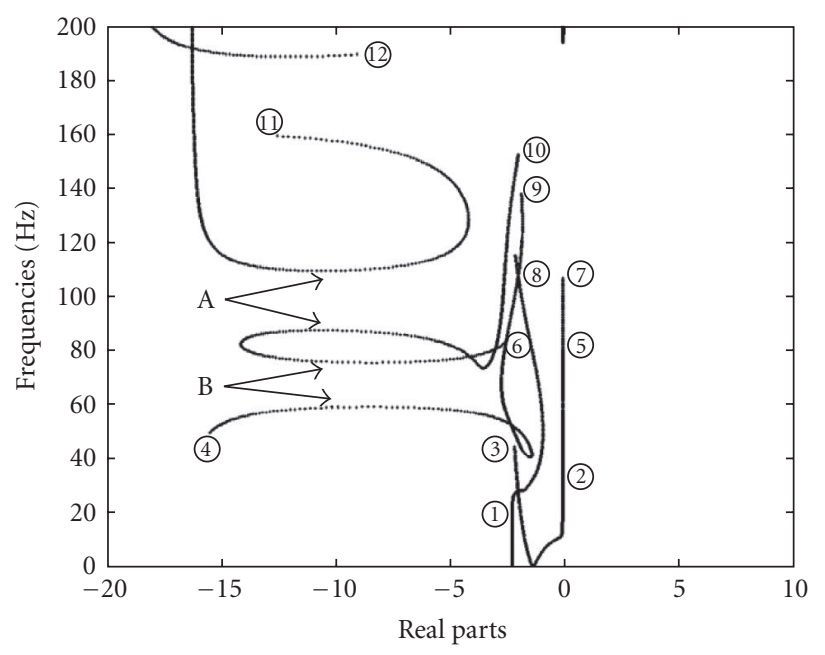

Figure 4: Complex plane diagram for a twenty-eight-bladed rotor with blades of one meter in length.

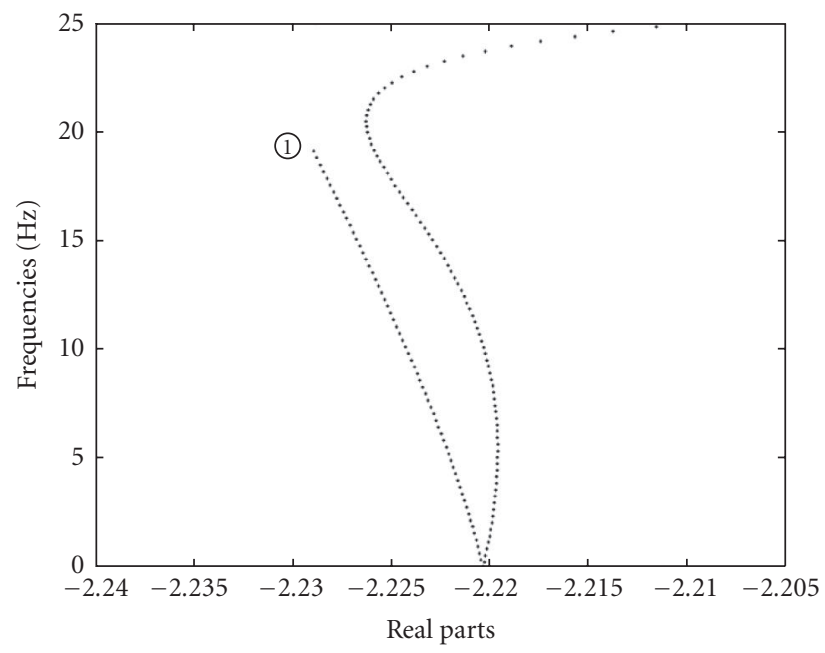

Figure 5: Zoom near extremity (1) in the complex plane diagram.

sloping lines are forward modes (precession motions in the same sense of rotation as the own rotation of the shaft) and the upward sloping ones are backward modes. The evolution of the blades' eigenfrequencies (starting at $31 \mathrm{~Hz}$ ), that is, their stiffening, is due to the centrifugal effects included in the prestress potential $v_{g_{\text {blade }}}$. Two loci separation phenomena $[12,13]$ appear, as illustrated by the points A and B on Figures 3 and 4 . When two eigenvalues loci approach each other, they either cross or do not cross; often in the latter case, even though the loci nearly intersect, in fact they do not but rather veer away from each other with high local curvature. During these veering phenomena, mode shapes and sense of rotation are switched between the eigenvalues that veer away from each other. The two particular cases shown in Figure 3 do not present high local curvature and their locations on the complex plane on Figure 4 are easy to find. In this particular simple case of loci separation, the evolution of the eigenvalues on both Figures 3 and 4 can be correlated.
The extremities of each curve are numbered in a round. Figures 5 and 6 are zooms of the extremities (1) and (2), respectively. The curves being "continuous," the eigenfrequencies at $\Omega=0$ RPM are not remarkable points. In this plane, loci separations look obviously like loops.

This system is stable as shown in Figure 4. However, in the particular case of a two-bladed rotor, instabilities can be observed as illustrated in Figures 7 and 8 showing a Campbell diagram and a decay rate plot (corresponding to the eigenvalues' real parts), respectively. Figure 9 shows the evolution of the eigenfrequencies in the complex plane. Loci separation phenomena appear and are more present than in the latter twenty eight-bladed rotor case.

In this case, two kinds of instabilities are observed. They both result from an inertial asymmetry of the system. The first and more common one is noted by (1) and occurs at the first critical speed (intersection with the abscissa axis). The second one, indicated by the marks $2 \mathrm{~b}, 2 \mathrm{c}$, and $2 \mathrm{~d}$, consists 


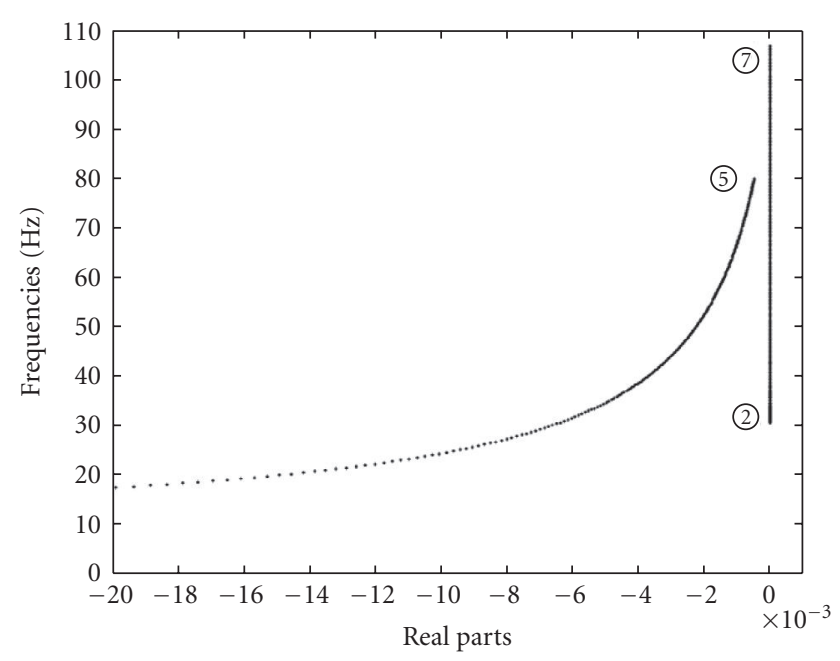

FIGURE 6: Zoom near extremity (2) in the complex plane diagram.

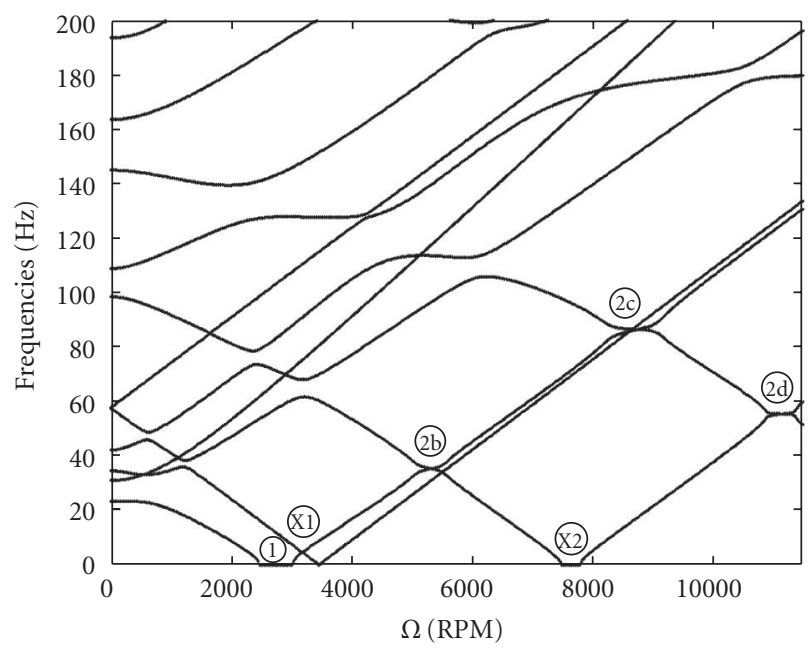

Figure 7: Campbell diagram for a two-bladed rotor.

in mode couplings, that is, two separated mode shapes at a rotational speed become two mode shapes having the same eigenfrequency but one of them being stable and the other being unstable with its amplitude increasing with respect to time. The points indicated by X1 and X2 do not show instabilities, but since the observed phenomenon is due to asymmetries, the length of the blades may influence mode couplings, and for sufficient length, may drive the two-bladed rotor unstable at these configurations.

Figure 10 shows a stability map for a two-bladed rotor as a function of its rotational speed and of its blade length, and Figure 11 shows the unstable frequencies associated. These two pictures confirm the two kinds of instabilities, the one noted (1) corresponding to $0 \mathrm{~Hz}$ (in the rotating frame) and the ones noted $2 \mathrm{a}, 2 \mathrm{~b}, 2 \mathrm{c}$, and $2 \mathrm{~d}$ corresponding to mode couplings. Figure 10 shows that up to $15 \mathrm{~cm}$ (in the case of these particular two blades), the asymmetry of the rotor is light enough to leave the system stable, but above this blade

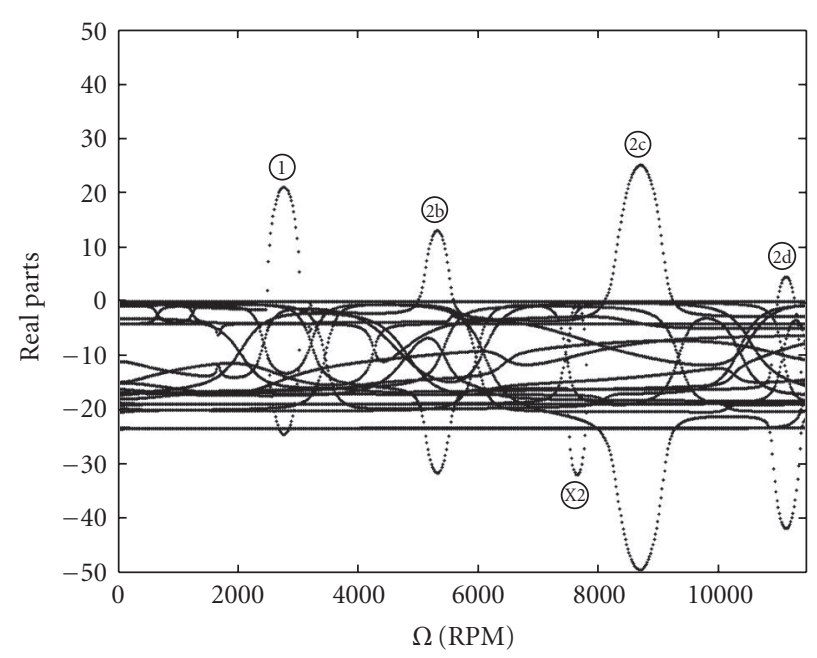

FIGURE 8: Decay rate plot for a two-bladed rotor.

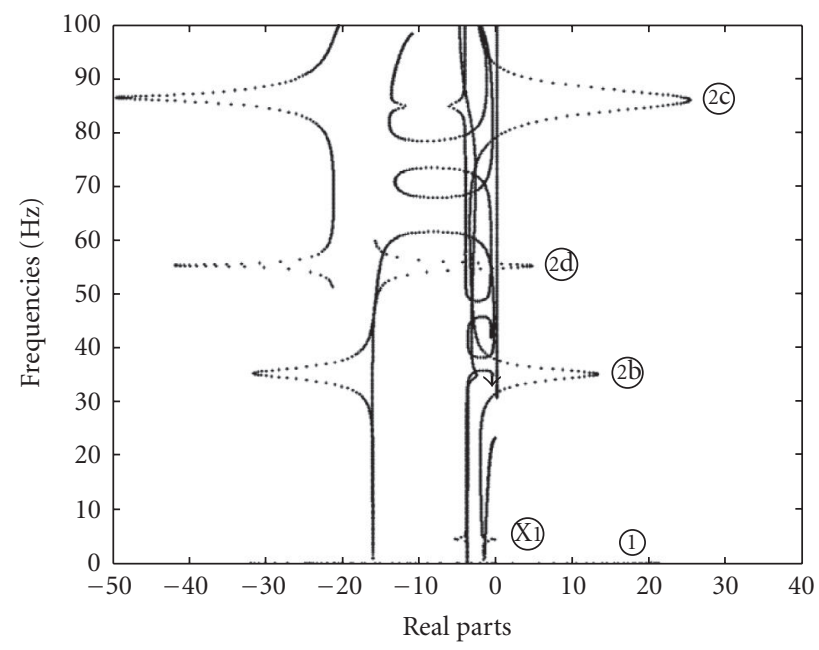

FIGURE 9: Complex plane diagram for a two-bladed rotor.

length, the rotor may experience several speed ranges where it is unstable. Only the unstable frequencies different from $0 \mathrm{~Hz}$ and corresponding to mode couplings can be seen in Figure 11, underlying the two kinds of instabilities. Thus, the configuration noted X1 on Figures 7 and 9 can be an unstable one if the blade length is up to $1.1 \mathrm{~m}$ and corresponds to the area noted $2 \mathrm{a}$ in Figure 10. It is the same phenomenon for the points noted X2 on Figures 7 and 8 which correspond to the area (1) just before 8000 RPM, in Figure 10, for blades longer than $1.1 \mathrm{~m}$.

As explained previously, these instabilities are mainly due to asymmetries of the system but, the flexibility of the blades can influence these phenomena. Figures 12 and 13 show the stability map and the unstable frequencies' evolution, respectively, in the case of a two-rigid-bladed rotor. The differences between this case and the latter concern mainly the first instability speed range. As a matter of fact, the unstable frequencies corresponding to mode couplings between 2000 RPM 


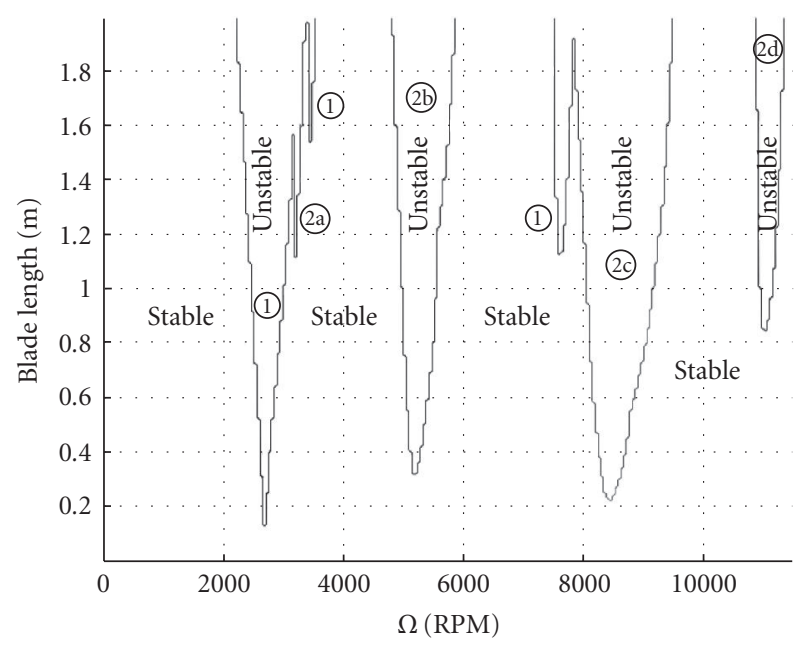

FIGURE 10: Stability map of a two-bladed rotor as a function of its blade length and its rotational speed.

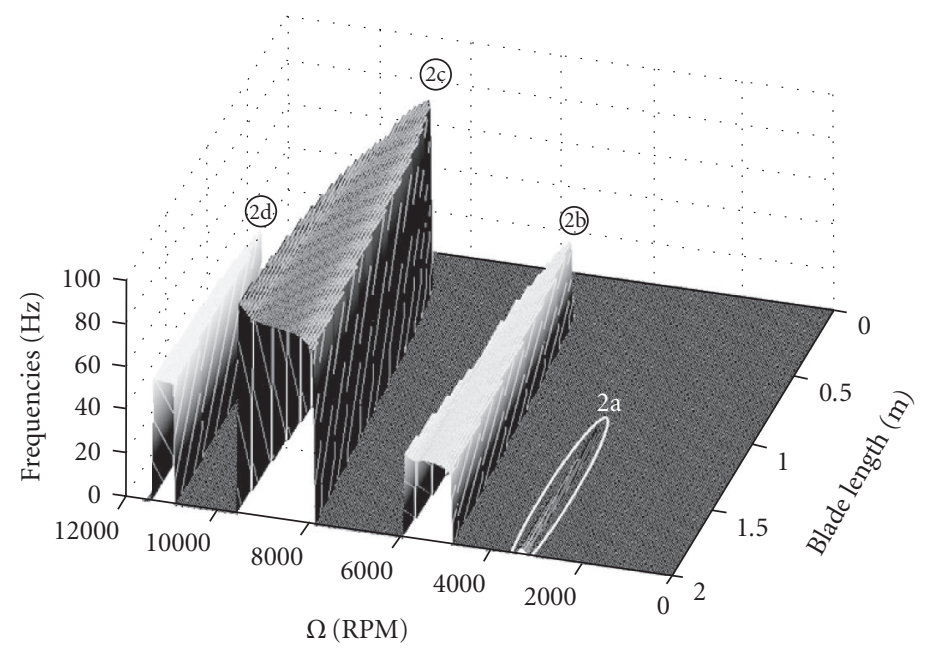

FIgURE 11: Unstable frequencies of a two-bladed rotor as a function of its blade length and its rotational speed.

and 4000 RPM are lower than in the flexible case and the blade length range concerned is shorter (see Figure 13, in white ellispe). Thus, in the case of a two-rigid-bladed rotor, the mass and inertia added by the two blades can, between 2000 RPM and 4000 RPM, lead to low-frequency modes couplings between the horizontal and the vertical mode shape of the rotor, if its blade length is between $1.1 \mathrm{~m}$ and $1.5 \mathrm{~m}$. In the case of flexible blades, if their eigenfrequencies are close to those of the shaft (as in the latter example), mode couplings can occur in this same speed range from a blade length of $1.1 \mathrm{~m}$ and involve mixed mode shapes.

Another parameter, the stagger angle (see Figure 14), can influence the occurrence of instabilities. All cases studied so far have been made with $\beta=60^{\circ}$. Figures 15 and 16 show the stability map and the unstable frequencies' evolution, respectively, as a function of the stagger angle of a two-flexible-bladed rotor of one meter in length. The two kinds of instabilities can also be seen here. It appears that the higher the stagger angle is, the shorter the unstable speed ranges are, as it could be though intuitively. However, unstable speed ranges can appear or disappear as a function of this stagger angle, showing the importance of this parameter.

\section{CONCLUSION}

A flexible fully bladed rotor has been modelled in the rotating frame using an energetic approach. Campbell diagrams have been plotted for this structure, what would not have been possible if the model had been made in the fixed frame. Then, its stability has been investigated, essentially by using complex plane diagrams. It has been shown that without rotating damping, the system may experience instabilities if it is strongly asymmetric, typically in the case of a twobladed rotor. In this case, zero hertz unstable speed ranges have been detected at the first critical speed as well as mode 


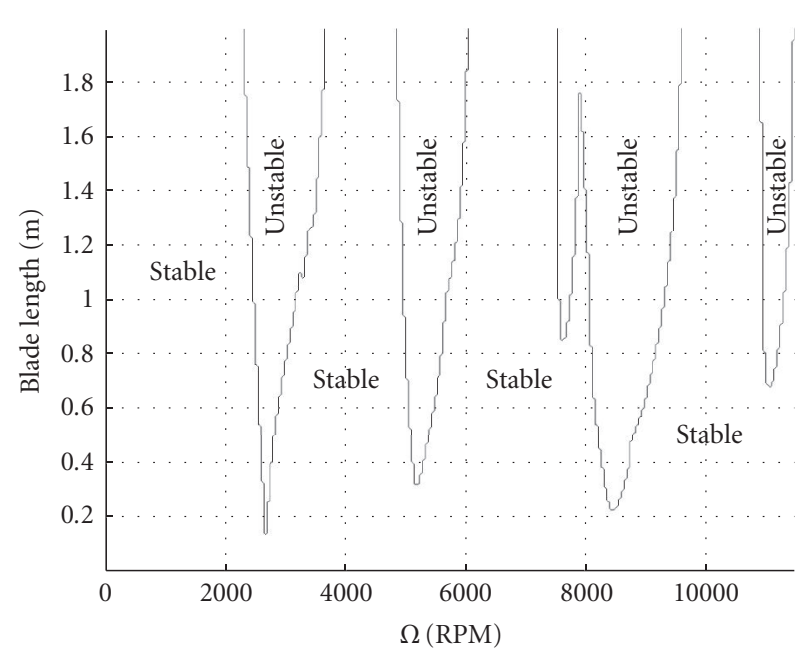

FIGURE 12: Stability map of a two-rigid-bladed rotor as a function of its blade length and its rotational speed.

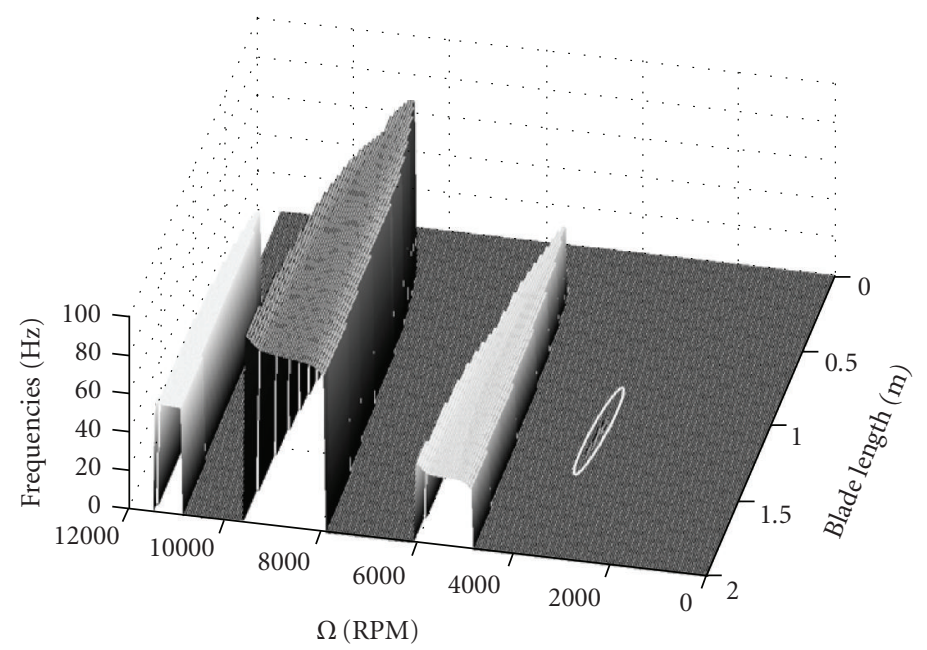

FIGURE 13: Unstable frequencies of a two-rigid-bladed rotor as a function of its blade length and its rotational speed.

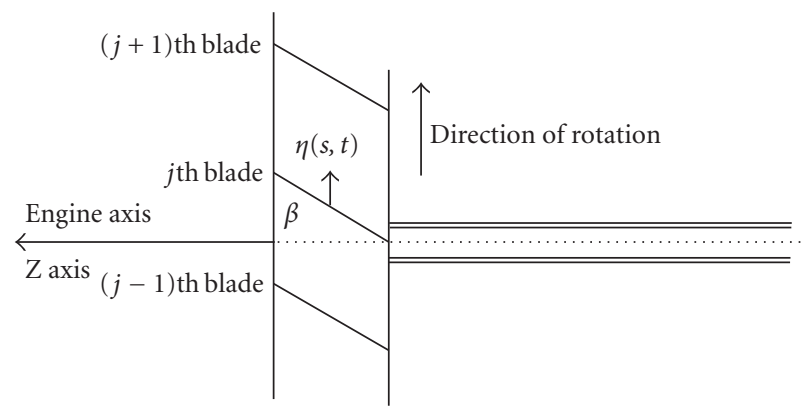

FIGURE 14: Up view of the bladed disk and the stagger angle $\beta$ of the blades.

couplings at frequencies different from zero hertz whether the blades are flexible or not. The influence of the stagger angle of the blades in the occurrences of instabilities has also been underlined. Thus, this model of a flexible bladed rotor appears to be sufficiently simple and complete from a phenomenological point of view to be used to design rotating machinery, to eliminate vibrations due to instabilities, and to study easily some complicated phenomenon in rotor dynamics as for instance travelling wave speed coincidence or blade-tip/casing contact. 


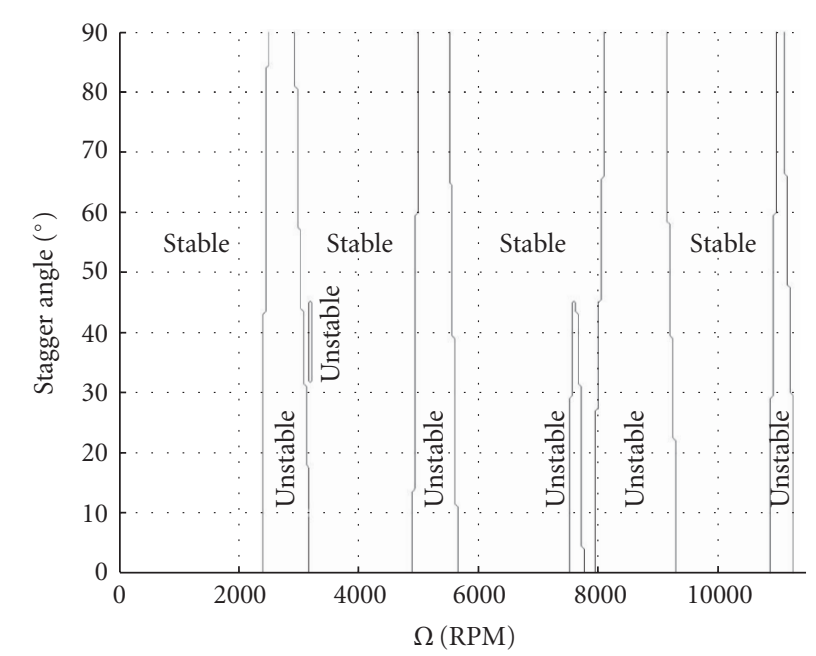

FIGURE 15: Stability map of a two-flexible-bladed rotor as a function of its stagger angle and its rotational speed.

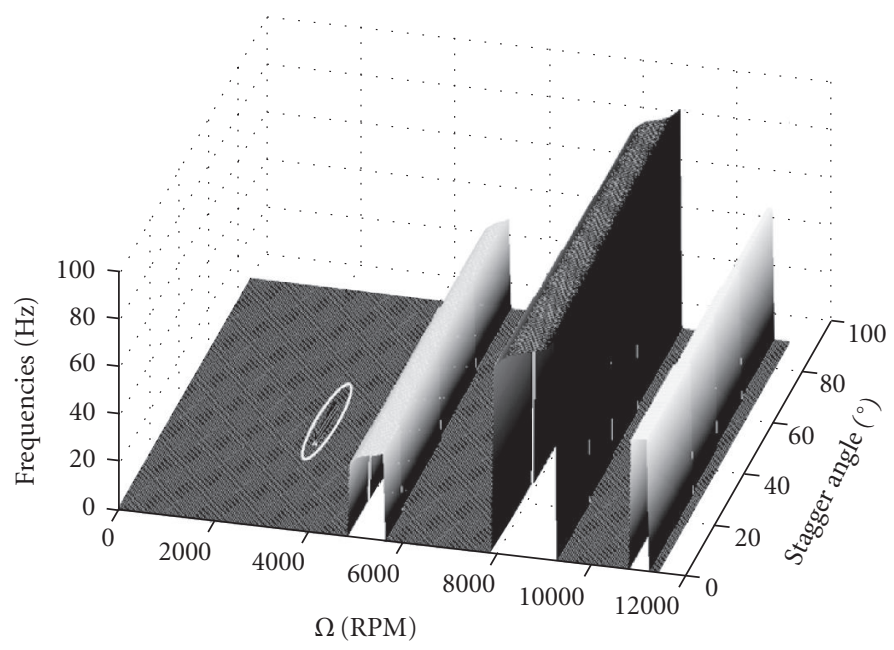

FIGURE 16: Unstable frequencies of a two-flexible-bladed rotor as a function of its stagger angle and its rotational speed.

\section{APPENDIX}

\section{A. EXPRESSIONS OF THE ENERGIES AND POTENTIALS OF THE MODEL'S ITEMS}

$$
\begin{aligned}
& T_{\text {blade }}=\int_{0}^{L} \frac{1}{2} \rho_{b} S_{b} \vec{V}_{S / R_{0}}^{2}(G) d s+\int_{0}^{L} \frac{1}{2} \vec{\Omega}_{S / R_{0}}^{T} \mathbf{I} \vec{\Omega}_{S / R_{0}} d s \\
&=\frac{1}{2} \int_{0}^{L} \rho_{b} S_{b}\left\{\dot{u}^{2}(l, t)+\dot{v}^{2}(l, t)+\dot{\eta}^{2}(s, t)\right. \\
& \quad-2 \dot{u}(l, t) \dot{\eta}(s, t) \cos \beta \sin \varphi_{j} \\
&\left.\quad+2 \dot{v}(l, t) \dot{\eta}(s, t) \cos \beta \cos \varphi_{j}\right\} d s \\
&+\frac{1}{2} \int_{0}^{L} \rho_{b} S_{b} \\
& \times\left\{\dot{u}^{\prime 2}(l, t)(s+r)^{2} \cos ^{2} \varphi_{j}\right. \\
&+\dot{v}^{\prime 2}(l, t)(s+r)^{2} \sin ^{2} \varphi_{j} \\
&+\left.2 \dot{u}^{\prime}(l, t) \dot{v}^{\prime}(l, t)(s+r)^{2} \cos \varphi_{j} \sin \varphi_{j}\right\} d s
\end{aligned}
$$

$$
\begin{gathered}
+\frac{1}{2} \int_{0}^{L} \rho_{b} S_{b}\left\{2 \dot{u}^{\prime}(l, t) \dot{\eta}(s, t)(s+r) \sin \beta \cos \varphi_{j}\right. \\
\left.+2 \dot{v}^{\prime}(l, t) \dot{\eta}(s, t)(s+r) \sin \beta \sin \varphi_{j}\right\} d s \\
+\frac{1}{2} \int_{0}^{L} \rho_{b} S_{b}\{2 \Omega[u(l, t) \dot{v}(l, t)-\dot{u}(l, t) v(l, t) \\
+\left(u(l, t) \cos \varphi_{j}+v(l, t) \sin \varphi_{j}\right) \\
\times \dot{\eta}(s, t) \cos \beta \\
-\left(\dot{u}(l, t) \cos \varphi_{j}+\dot{v}(l, t) \sin \varphi_{j}\right) \\
\times \eta(s, t) \cos \beta]\} d s \\
+\frac{1}{2} \int_{0}^{L} \rho_{b} S_{b} \Omega^{2}\left\{u^{2}(l, t)+v^{2}(l, t)+\eta^{2}(s, t) \cos ^{2} \beta\right. \\
-2 u(l, t) \eta(s, t) \cos \beta \sin \varphi_{j} \\
\left.+2 v(l, t) \eta(s, t) \cos \beta \cos \varphi_{j}\right\} d s \\
+\frac{1}{2} \int_{0}^{L} \rho_{b} S_{b} \\
\times\left\{2(s+r) \Omega^{2}\left[u(l, t) \cos \varphi_{j}+v(l, t) \sin \varphi_{j}\right]\right.
\end{gathered}
$$




$$
\begin{aligned}
& \begin{array}{l}
+2(s+r) \Omega\left[\dot{v}(l, t) \cos \varphi_{j}-\dot{u}(l, t) \sin \varphi_{j}\right] \\
\left.+\Omega^{2}(s+r)^{2}\right\} d s
\end{array} \quad+\frac{1}{2} \int_{0}^{L} \rho_{b} I_{b} \Omega^{2} \\
& +\frac{1}{2} \int_{0}^{L} \rho_{b} S_{b} 2(s+r)^{2} \Omega \\
& \times\left\{\dot{u}^{\prime}(l, t) u^{\prime}(l, t) \cos \varphi_{j} \sin \varphi_{j}\right. \\
& -\dot{v}^{\prime}(l, t) v^{\prime}(l, t) \cos \varphi_{j} \sin \varphi_{j} \\
& -\dot{u}^{\prime}(l, t) v^{\prime}(l, t) \cos ^{2} \varphi_{j} \\
& \left.-\dot{v}^{\prime}(l, t) u^{\prime}(l, t) \cos ^{2} \varphi_{j}\right\} d s \\
& +\frac{1}{2} \int_{0}^{L} \rho_{b} S_{b} 2(s+r) \Omega \\
& \times\left\{\left[\dot{\eta}(s, t) u^{\prime}(l, t)+\eta(s, t) \dot{u}^{\prime}(l, t)\right]\right. \\
& \times \sin \beta \sin \varphi_{j} \\
& -\left[\dot{\eta}(s, t) v^{\prime}(l, t)+\eta(s, t) \dot{v}^{\prime}(l, t)\right] \\
& \left.\times \sin \beta \cos \varphi_{j}+\dot{\eta}(s, t) \cos \beta\right\} d s \\
& -\frac{1}{2} \int_{0}^{L} \rho_{b} S_{b} \Omega^{2}(s+r)^{2} \\
& \times\left\{u^{\prime 2}(l, t) \cos ^{2} \varphi_{j}+v^{\prime 2}(l, t) \sin ^{2} \varphi_{j}\right. \\
& \left.+2 u^{\prime}(l, t) v^{\prime}(l, t) \cos \varphi_{j} \sin \varphi_{j}\right\} d s \\
& -\frac{1}{2} \int_{0}^{L} \rho_{b} S_{b} \Omega^{2}(s+r) \\
& \times\left\{2 \eta(s, t) v^{\prime}(l, t) \sin \beta \sin \varphi_{j}\right. \\
& \left.+2 \eta(s, t) u^{\prime}(l, t) \sin \beta \cos \varphi_{j}\right\} d s \\
& +\frac{1}{2} \int_{0}^{L} \rho_{b} I_{b}\left\{\dot{u}^{\prime 2}(l, t) \sin ^{2} \beta \cos ^{2} \varphi_{j}\right. \\
& +\dot{v}^{\prime 2}(l, t) \sin ^{2} \beta \sin ^{2} \varphi_{j}+\dot{\eta}^{\prime 2}(s, t) \\
& +2 \dot{u}^{\prime}(l, t) \dot{\eta}^{\prime}(s, t) \sin \beta \cos \varphi_{j} \\
& \left.+2 \dot{v}^{\prime}(l, t) \dot{\eta}^{\prime}(s, t) \sin \beta \sin \varphi_{j}\right\} d s \\
& +\frac{1}{2} \int_{0}^{L} \rho_{b} I_{b}\left\{2 \dot{u}^{\prime}(l, t) \dot{v}^{\prime}(l, t) \sin ^{2} \beta \cos \varphi_{j} \sin \varphi_{j}\right\} d s \\
& +\frac{1}{2} \int_{0}^{L} \rho_{b} I_{b} 2 \Omega \\
& \times\left\{\dot{v}^{\prime}(l, t)\left(u^{\prime}(l, t) \sin \varphi_{j}-v^{\prime}(l, t) \cos \varphi_{j}\right)\right. \\
& \times \sin ^{2} \beta \sin \varphi_{j}+\dot{v}^{\prime}(l, t) \sin \beta \cos \beta \sin \varphi_{j} \\
& \left.-\dot{v}^{\prime}(l, t) u^{\prime}(l, t) \cos ^{2} \beta\right\} d s \\
& +\frac{1}{2} \int_{0}^{L} \rho_{b} I_{b} 2 \Omega \\
& \times\left\{\dot{u}^{\prime}(l, t)\left(u^{\prime}(l, t) \sin \varphi_{j}-v^{\prime}(l, t) \cos \varphi_{j}\right)\right. \\
& \times \sin ^{2} \beta \cos \varphi_{j} \\
& \left.+\dot{u}^{\prime}(l, t) \sin \beta \cos \beta \cos \varphi_{j}\right\} d s \\
& +\frac{1}{2} \int_{0}^{L} \rho_{b} I_{b} 2 \Omega \\
& \times\left\{\dot{\eta}^{\prime}(s, t) \cos \beta+\dot{\eta}^{\prime}(s, t) u^{\prime}(l, t) \sin \beta \sin \varphi_{j}\right. \\
& \left.-\dot{\eta}^{\prime}(s, t) v^{\prime}(l, t) \sin \beta \cos \varphi_{j}\right\} d s \\
& \times\left\{u^{\prime 2}(l, t) \sin ^{2} \beta \sin ^{2} \varphi_{j}-u^{\prime 2}(l, t) \cos ^{2} \beta\right. \\
& +v^{\prime 2}(l, t) \sin ^{2} \beta \cos ^{2} \varphi_{j} \\
& \left.-v^{\prime 2}(l, t) \cos ^{2} \beta+\cos ^{2} \beta\right\} d s \\
& +\frac{1}{2} \int_{0}^{L} \rho_{b} I_{b} 2 \Omega^{2} \\
& \times\left\{u^{\prime}(l, t) \sin \beta \cos \beta \sin \varphi_{j}\right. \\
& -v^{\prime}(l, t) \cos \beta \sin \beta \cos \varphi_{j} \\
& \left.-u^{\prime}(l, t) v^{\prime}(l, t) \sin ^{2} \beta \cos \varphi_{j} \sin \varphi_{j}\right\} d s \\
& +\frac{1}{2} \int_{0}^{L} \rho_{b} I_{y} \\
& \times\left\{\dot{u}^{\prime 2}(l, t) \cos ^{2} \beta \cos ^{2} \varphi_{j}\right. \\
& +\dot{v}^{\prime 2}(l, t) \cos ^{2} \beta \sin ^{2} \varphi_{j} \\
& \left.+2 \dot{u}^{\prime}(l, t) \dot{v}^{\prime}(l, t) \cos ^{2} \beta \sin \varphi_{j} \cos \varphi_{j}\right\} d s \\
& +\frac{1}{2} \int_{0}^{L} \rho_{b} I_{y} 2 \Omega \\
& \times\left\{\dot{v}^{\prime}(l, t)\left(u^{\prime}(l, t) \sin \varphi_{j}-v^{\prime}(l, t) \cos \varphi_{j}\right)\right. \\
& \times \cos ^{2} \beta \sin \varphi_{j}-\dot{v}^{\prime}(l, t) \sin \beta \cos \beta \sin \varphi_{j} \\
& \left.-\dot{v}^{\prime}(l, t) u^{\prime}(l, t) \sin ^{2} \beta\right\} d s \\
& +\frac{1}{2} \int_{0}^{L} \rho_{b} I_{y} 2 \Omega \\
& \times\left\{\dot{u}^{\prime}(l, t)\left(u^{\prime}(l, t) \sin \varphi_{j}-v^{\prime}(l, t) \cos \varphi_{j}\right)\right. \\
& \times \cos ^{2} \beta \cos \varphi_{j} \\
& \left.-\dot{u}^{\prime}(l, t) \sin \beta \cos \beta \cos \varphi_{j}\right\} d s \\
& +\frac{1}{2} \int_{0}^{L} \rho_{b} I_{y} 2 \Omega\left\{\eta^{\prime}(s, t) \dot{u}^{\prime}(l, t) \sin \beta \sin \varphi_{j}\right. \\
& \left.-\eta^{\prime}(s, t) \dot{v}^{\prime}(l, t) \sin \beta \cos \varphi_{j}\right\} d s \\
& +\frac{1}{2} \int_{0}^{L} \rho_{b} I_{y} \Omega^{2} \\
& \times\left\{u^{\prime 2}(l, t) \cos ^{2} \beta \sin ^{2} \varphi_{j}-u^{\prime 2}(l, t) \sin ^{2} \beta\right. \\
& +v^{\prime 2}(l, t) \cos ^{2} \beta \cos ^{2} \varphi_{j}-v^{\prime 2}(l, t) \sin ^{2} \beta \\
& \left.-\eta^{\prime 2}(s, t) \sin ^{2} \beta+\sin ^{2} \beta\right\} d s \\
& -\frac{1}{2} \int_{0}^{L} \rho_{b} I_{y} 2 \Omega^{2} \\
& \times\left\{u^{\prime}(l, t) \sin \beta \cos \beta \sin \varphi_{j}\right. \\
& -v^{\prime}(l, t) \cos \beta \sin \beta \cos \varphi_{j} \\
& \left.+u^{\prime}(l, t) v^{\prime}(l, t) \cos ^{2} \beta \cos \varphi_{j} \sin \varphi_{j}\right\} d s \\
& -\frac{1}{2} \int_{0}^{L} \rho_{b} I_{y} 2 \Omega^{2}\left\{\eta^{\prime}(s, t) u^{\prime}(l, t) \sin \beta \cos \varphi_{j}\right. \\
& \left.+\eta^{\prime}(s, t) v^{\prime}(l, t) \sin \beta \sin \varphi_{j}\right\} d s \\
& +\frac{1}{2} \int_{0}^{L} \rho_{b} I_{z}\left\{\dot{u}^{\prime 2}(l, t) \sin ^{2} \varphi_{j}+\dot{v}^{\prime 2}(l, t) \cos ^{2} \varphi_{j}\right. \\
& \left.-2 \dot{u}^{\prime}(l, t) \dot{v}^{\prime}(l, t) \sin \varphi_{j} \cos \varphi_{j}\right\} d s
\end{aligned}
$$




$$
\begin{aligned}
& +\frac{1}{2} \int_{0}^{L} \rho_{b} I_{z} 2 \Omega \\
& \times\left\{\dot{v}^{\prime}(l, t)\left(u^{\prime}(l, t) \cos \varphi_{j}+v^{\prime}(l, t) \sin \varphi_{j}\right)\right. \\
& \left.\times \cos \varphi_{j}+\dot{v}^{\prime}(l, t) \eta^{\prime}(s, t) \sin \beta \cos \varphi_{j}\right\} d s \\
& -\frac{1}{2} \int_{0}^{L} \rho_{b} I_{z} 2 \Omega \\
& \times\left\{\dot{u}^{\prime}(l, t)\left(u^{\prime}(l, t) \cos \varphi_{j}+v^{\prime}(l, t) \sin \varphi_{j}\right)\right. \\
& \left.\times \sin \varphi_{j}+\dot{u}^{\prime}(l, t) \eta^{\prime}(s, t) \sin \beta \sin \varphi_{j}\right\} d s \\
& +\frac{1}{2} \int_{0}^{L} \rho_{b} I_{z} \Omega^{2} \\
& \times\left\{u^{\prime 2}(l, t) \cos ^{2} \varphi_{j}\right. \\
& \left.+v^{\prime 2}(l, t) \sin ^{2} \varphi_{j}+\eta^{\prime 2}(s, t) \sin ^{2} \beta\right\} d s \\
& +\frac{1}{2} \int_{0}^{L} \rho_{b} I_{z} 2 \Omega^{2}\left\{\eta^{\prime}(s, t) u^{\prime}(l, t) \sin \beta \cos \varphi_{j}\right. \\
& +\eta^{\prime}(s, t) v^{\prime}(l, t) \sin \beta \sin \varphi_{j} \\
& \left.+u^{\prime}(l, t) v^{\prime}(l, t) \cos \varphi_{j} \sin \varphi_{j}\right\} d s, \\
& v_{\text {int }_{\text {blade }}}=\frac{1}{2} \int_{0}^{L} E_{b} I_{b}\left(\eta^{\prime \prime}(s, t)\right)^{2} d s, \\
& F_{d_{\text {blade }}}=\frac{1}{2} \int_{0}^{L} \eta_{b} E_{b} I_{b}\left(\dot{\eta}^{\prime \prime}(s, t)\right)^{2} d s, \\
& v_{g_{\text {blade }}}=\frac{1}{2} \int_{0}^{L} \rho_{b} A_{b} \Omega^{2}\left[\frac{R^{2}-(s+r)^{2}}{2}\right] \eta^{\prime 2}(s, t) d s, \\
& T_{\text {shaft }}=\frac{1}{2} \int_{0}^{l} \rho_{s} A_{s}\left(\dot{u}^{2}(z, t)+\dot{v}^{2}(z, t)\right) d z \\
& +\int_{0}^{l} \rho_{s} A_{s} \Omega\{u(z, t) \dot{v}(z, t)-\dot{u}(z, t) v(z, t)\} d z \\
& +\frac{1}{2} \int_{0}^{l} \rho_{s} A_{s} \Omega^{2}\left(u^{2}(z, t)+v^{2}(z, t)\right) d z \\
& +\frac{1}{2} \int_{0}^{l} \rho_{s}\left\{I_{s_{X}} \dot{v}^{\prime 2}(z, t)+I_{s_{Y}} \dot{u}^{\prime 2}(z, t)\right\} d z \\
& +\int_{0}^{l} \rho_{s} \Omega\left\{I_{s_{X}} \dot{v}^{\prime}(z, t) u^{\prime}(z, t)-I_{S_{Y}} \dot{u}^{\prime}(z, t) v^{\prime}(z, t)\right\} d z \\
& +\frac{1}{2} \int_{0}^{l} \rho_{s} \Omega^{2}\left\{\left(I_{S_{Y}}-I_{s}^{\mathrm{pol}}\right) v^{\prime 2}(z, t)\right. \\
& \left.+\left(I_{s_{X}}-I_{s}^{\mathrm{pol}}\right) u^{\prime 2}(z, t)\right\} d z \\
& +\frac{1}{2} \int_{0}^{l} \rho_{s} I_{s}^{\mathrm{pol}}\left(\Omega^{2}-2 \Omega \dot{v}^{\prime}(z, t) u^{\prime}(z, t)\right) d z \\
& +\frac{1}{2} M_{D}\left(\dot{u}^{2}(l, t)+\dot{v}^{2}(l, t)\right) \\
& +M_{D} \Omega\{u(l, t) \dot{v}(l, t)-\dot{u}(l, t) v(l, t)\} \\
& +\frac{1}{2} M_{D} \Omega^{2}\left(u^{2}(l, t)+v^{2}(l, t)\right) \\
& +\frac{1}{2}\left\{J_{D_{X}} \dot{v}^{\prime 2}(l, t)+J_{D_{Y}} \dot{u}^{\prime 2}(l, t)\right\} \\
& +\Omega\left\{J_{D_{X}} \dot{v}^{\prime}(l, t) u^{\prime}(l, t)-J_{D_{Y}} \dot{u}^{\prime}(l, t) v^{\prime}(l, t)\right\} \\
& +\frac{1}{2} \Omega^{2}\left\{\left(J_{D_{Y}}-J_{D}^{\mathrm{pol}}\right) v^{\prime 2}(l, t)+\left(J_{D_{X}}-J_{D}^{\mathrm{pol}}\right) u^{\prime 2}(l, t)\right\} \\
& +\frac{1}{2} J_{D}^{\mathrm{pol}}\left(\Omega^{2}-2 \Omega \dot{v}^{\prime}(l, t) u^{\prime}(l, t)\right)
\end{aligned}
$$

$$
\begin{aligned}
v_{\text {int }_{\text {shaft }}=} & \frac{1}{2} \int_{0}^{l} E_{s} I_{s}\left(u^{\prime \prime 2}(z, t)+v^{\prime \prime 2}(z, t)\right) d z \\
F_{d_{\text {shaft }}=} & \frac{1}{2} \int_{0}^{l} \eta_{s} E_{s} I_{s}\left(\dot{u}^{\prime \prime 2}(z, t)+\dot{v}^{\prime \prime 2}(z, t)\right) d z \\
v_{\text {bearings }}= & \sum_{i=1}^{N b} \frac{1}{2}\left[S_{x x}^{b}\left\{u\left(z_{i}, t\right) \cos \Omega t-v\left(z_{i}, t\right) \sin \Omega t\right\}^{2}\right. \\
& \left.+S_{y y}^{b}\left\{u\left(z_{i}, t\right) \sin \Omega t+v\left(z_{i}, t\right) \cos \Omega t\right\}^{2}\right] \\
& +\sum_{i=1}^{N b} \frac{1}{2}\left[B_{x x}^{b}\left\{u^{\prime}\left(z_{i}, t\right) \cos \Omega t-v^{\prime}\left(z_{i}, t\right) \sin \Omega t\right\}^{2}\right. \\
& \left.+B_{y y}^{b}\left\{u^{\prime}\left(z_{i}, t\right) \sin \Omega t+v^{\prime}\left(z_{i}, t\right) \cos \Omega t\right\}^{2}\right], \\
F_{d_{\text {bearings }}=} & \sum_{i=1}^{N b} \frac{1}{2}\left[D _ { x x } ^ { b } \left\{\dot{u}\left(z_{i}, t\right) \cos \Omega t-u\left(z_{i}, t\right) \Omega \sin \Omega t\right.\right. \\
& +\sum_{i=1} \frac{1}{2}\left[D_{y y}^{b}\left\{\dot{v}\left(z_{i}, t\right) \sin \Omega t-v\left(z_{i}, t\right) \Omega \cos \Omega t\right\}^{2}\right] \\
& \left.\left.+\dot{v}\left(z_{i}, t\right) \cos \Omega t-v\left(z_{i}, t\right) \Omega \sin \Omega t\right\}^{2}\right] .
\end{aligned}
$$

\section{B. EXPRESSIONS OF THE MASS, DAMPING, AND STIFFNESS MATRICES FOR THE ROTOR}

Considering that $m \in\left[1, m_{\mathrm{tot}}\right], q \in\left[1, m_{\mathrm{tot}}\right], c \in\left[3, n_{\mathrm{tot}}+2\right]$, $p \in\left[3, n_{\text {tot }}+2\right]$, and $j \in\left[1, N_{\text {tot }}\right]$, the coefficients of $\mathbf{M}_{\text {rotor }}$, $\widetilde{\mathbf{K}}_{\text {rotor }}$, and $\widetilde{\mathbf{C}}_{\text {rotor }}$ are given by

$$
\begin{aligned}
& \mathbf{M}_{\text {rotor }}(1,1)=M_{s}+M_{D}+N_{\text {tot }} M_{b}, \\
& \mathbf{M}_{\text {rotor }}(1,2)=0, \\
& \mathbf{M}_{\text {rotor }}(1,2 m+1) \\
& \quad=\int_{0}^{l} \rho_{s} A_{s} W_{m}(z) d z+M_{D} W_{m}(l)+N_{\text {tot }} M_{b} W_{m}(l), \\
& \mathbf{M}_{\text {rotor }}(1,2 m+2)=0, \\
& \quad \mathbf{M}_{\text {rotor }}\left(1,2 m_{\text {tot }}+c+(j-1) n_{\text {tot }}\right) \\
& \quad=-\int_{0}^{L} \rho_{b} A_{b} Y_{c-2}(s) d s \cos \beta \sin \varphi_{j}, \\
& \mathbf{M}_{\text {rotor }}(2,1)=0, \\
& \mathbf{M}_{\text {rotor }}(2,2)=M_{s}+M_{D}+N_{\text {tot }} M_{b}, \\
& \mathbf{M}_{\text {rotor }}(2,2 m+1)=0, \\
& \mathbf{M}_{\text {rotor }}(2,2 m+2) \\
& \quad=\int_{0}^{l} \rho_{s} A_{s} W_{m}(z) d z+M_{D} W_{m}(l)+N_{\text {tot }} M_{b} W_{m}(l), \\
& \quad \mathbf{M}_{\text {rotor }}\left(2,2 m_{\text {tot }}+c+(j-1) n_{\text {tot }}\right) \\
& \quad \int_{0}^{L} \rho_{b} A_{b} Y_{c-2}(s) d s \cos \beta \cos \varphi_{j},
\end{aligned}
$$




$$
\begin{aligned}
& \mathbf{M}_{\text {rotor }}(2 m+1,1) \\
& =\int_{0}^{l} \rho_{s} A_{s} W_{m}(z) d z+M_{D} W_{m}(l)+N_{\text {tot }} M_{b} W_{m}(l), \\
& \mathbf{M}_{\text {rotor }}(2 m+1,2)=0 \text {, } \\
& \mathbf{M}_{\text {rotor }}(2 m+1,2 q+1) \\
& =\int_{0}^{l} \rho_{s} A_{s} W_{q}(z) W_{m}(z) d z+\int_{0}^{l} \rho_{s} I_{s_{y}} W_{q}^{\prime}(z) W_{m}^{\prime}(z) d z \\
& +M_{D} W_{q}(l) W_{m}(l)+J_{D_{y}} W_{q}^{\prime}(l) W_{m}^{\prime}(l) \\
& +N_{\text {tot }} M_{b} W_{q}(l) W_{m}(l) \\
& +\sum_{j=1}^{N_{\mathrm{tot}}} M_{b}\left\{r^{2}+L r+\frac{L^{2}}{3}\right\} W_{q}^{\prime}(l) W_{m}^{\prime}(l) \cos ^{2} \varphi_{j} \\
& +\sum_{j=1}^{N_{\text {tot }}} L \rho_{b} I_{b} \sin ^{2} \beta \cos ^{2} \varphi_{j} W_{q}^{\prime}(l) W_{m}^{\prime}(l) \\
& +\sum_{j=1}^{N_{\text {tot }}} L \rho_{b} I_{y} \cos ^{2} \beta \cos ^{2} \varphi_{j} W_{q}^{\prime}(l) W_{m}^{\prime}(l) \\
& +\sum_{j=1}^{N_{\text {tot }}} L \rho_{b} I_{z} \sin ^{2} \varphi_{j} W_{q}^{\prime}(l) W_{m}^{\prime}(l) \\
& \mathbf{M}_{\text {rotor }}(2 m+1,2 q+2) \\
& =\sum_{j=1}^{N_{\mathrm{tot}}} M_{b}\left\{r^{2}+L r+\frac{L^{2}}{3}\right\} W_{q}^{\prime}(l) W_{m}^{\prime}(l) \sin \varphi_{j} \cos \varphi_{j} \\
& +\sum_{j=1}^{N_{\mathrm{tot}}} L \rho_{b} I_{b} \sin ^{2} \beta \cos \varphi_{j} \sin \varphi_{j} W_{q}^{\prime}(l) W_{m}^{\prime}(l) \\
& +\sum_{j=1}^{N_{\text {tot }}} L \rho_{b} I_{y} \cos ^{2} \beta \cos \varphi_{j} \sin \varphi_{j} W_{q}^{\prime}(l) W_{m}^{\prime}(l) \\
& -\sum_{j=1}^{N_{\text {tot }}} L \rho_{b} I_{z} \cos \varphi_{j} \sin \varphi_{j} W_{q}^{\prime}(l) W_{m}^{\prime}(l), \\
& \mathbf{M}_{\text {rotor }}\left(2 m+1,2 m_{\text {tot }}+c+(j-1) n_{\text {tot }}\right) \\
& =-\int_{0}^{L} \rho_{b} A_{b} Y_{c-2}(s) d s W_{m}(l) \cos \beta \sin \varphi_{j} \\
& +\int_{0}^{L} \rho_{b} A_{b} Y_{c-2}(s)(s+r) d s W_{m}^{\prime}(l) \sin \beta \cos \varphi_{j} \\
& +\int_{0}^{L} \rho_{b} I_{b} Y_{c-2}^{\prime}(s) d s W_{m}^{\prime}(l) \sin \beta \cos \varphi_{j}, \\
& \mathbf{M}_{\text {rotor }}(2 m+2,1)=0 \text {, } \\
& \mathbf{M}_{\text {rotor }}(2 m+2,2) \\
& =\int_{0}^{l} \rho_{s} A_{s} W_{m}(z) d z+M_{D} W_{m}(l)+N_{\mathrm{tot}} M_{b} W_{m}(l), \\
& \mathbf{M}_{\text {rotor }}(2 m+2,2 q+1) \\
& =\sum_{j=1}^{N_{\mathrm{tot}}} M_{b}\left\{r^{2}+L r+\frac{L^{2}}{3}\right\} W_{q}^{\prime}(l) W_{m}^{\prime}(l) \sin \varphi_{j} \cos \varphi_{j} \\
& +\sum_{j=1}^{N_{\text {tot }}} L \rho_{b} I_{b} \sin ^{2} \beta \cos \varphi_{j} \sin \varphi_{j} W_{q}^{\prime}(l) W_{m}^{\prime}(l) \\
& +\sum_{j=1}^{N_{\text {tot }}} L \rho_{b} I_{y} \cos ^{2} \beta \cos \varphi_{j} \sin \varphi_{j} W_{q}^{\prime}(l) W_{m}^{\prime}(l) \\
& -\sum_{j=1}^{N_{\text {tot }}} L \rho_{b} I_{z} \cos \varphi_{j} \sin \varphi_{j} W_{q}^{\prime}(l) W_{m}^{\prime}(l), \\
& \mathbf{M}_{\text {rotor }}(2 m+2,2 q+2) \\
& =\int_{0}^{l} \rho_{s} A_{s} W_{q}(z) W_{m}(z) d z+\int_{0}^{l} \rho_{s} I_{s_{x}} W_{q}^{\prime}(z) W_{m}^{\prime}(z) d z \\
& +M_{D} W_{q}(l) W_{m}(l)+J_{D_{x}} W_{q}^{\prime}(l) W_{m}^{\prime}(l) \\
& +N_{\text {tot }} M_{b} W_{q}(l) W_{m}(l) \\
& +\sum_{j=1}^{N_{\text {tot }}} M_{b}\left\{r^{2}+L r+\frac{L^{2}}{3}\right\} W_{q}^{\prime}(l) W_{m}^{\prime}(l) \sin ^{2} \varphi_{j} \\
& +\sum_{j=1}^{N_{\text {tot }}} L \rho_{b} I_{b} \sin ^{2} \beta \sin ^{2} \varphi_{j} W_{q}^{\prime}(l) W_{m}^{\prime}(l) \\
& +\sum_{j=1}^{N_{\text {tot }}} L \rho_{b} I_{y} \cos ^{2} \beta \sin ^{2} \varphi_{j} W_{q}^{\prime}(l) W_{m}^{\prime}(l) \\
& +\sum_{j=1}^{N_{\text {tot }}} L \rho_{b} I_{z} \cos ^{2} \varphi_{j} W_{q}^{\prime}(l) W_{m}^{\prime}(l), \\
& \mathbf{M}_{\text {rotor }}\left(2 m+2,2 m_{\text {tot }}+c+(j-1) n_{\text {tot }}\right) \\
& =\int_{0}^{L} \rho_{b} A_{b} Y_{c-2}(s) d s W_{m}(l) \cos \beta \cos \varphi_{j} \\
& +\int_{0}^{L} \rho_{b} A_{b} Y_{c-2}(s)(s+r) d s W_{m}^{\prime}(l) \sin \beta \sin \varphi_{j} \\
& +\int_{0}^{L} \rho_{b} I_{b} Y_{c-2}^{\prime}(s) d s W_{m}^{\prime}(l) \sin \beta \sin \varphi_{j}, \\
& \mathbf{M}_{\text {rotor }}\left(2 m_{\mathrm{tot}}+p+(j-1) n_{\mathrm{tot}}, 1\right) \\
& =-\int_{0}^{L} \rho_{b} A_{b} Y_{p-2}(s) d s \cos \beta \sin \varphi_{j}, \\
& \mathbf{M}_{\text {rotor }}\left(2 m_{\mathrm{tot}}+p+(j-1) n_{\mathrm{tot}}, 2\right) \\
& =\int_{0}^{L} \rho_{b} A_{b} Y_{p-2}(s) d s \cos \beta \cos \varphi_{j},
\end{aligned}
$$




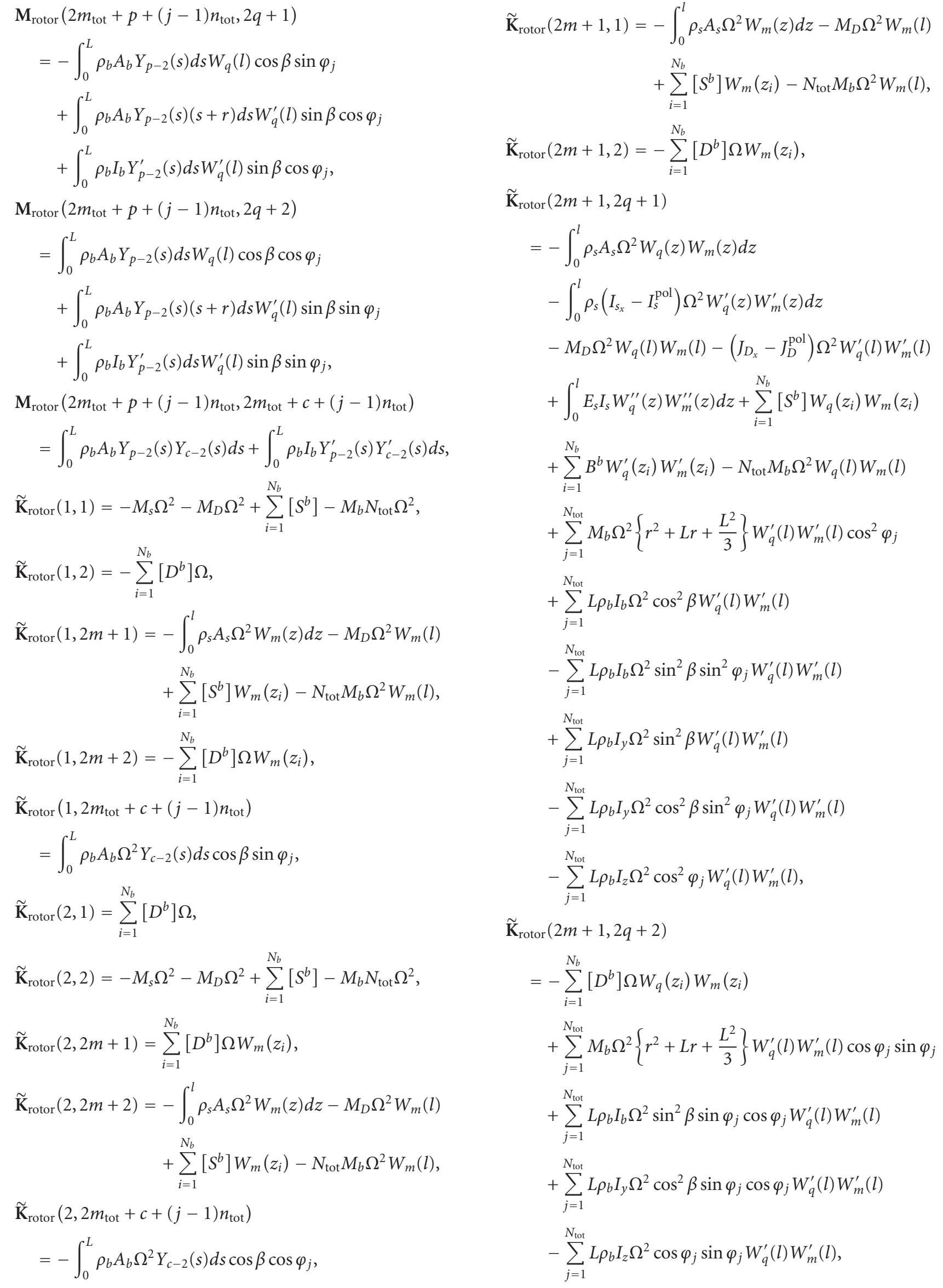

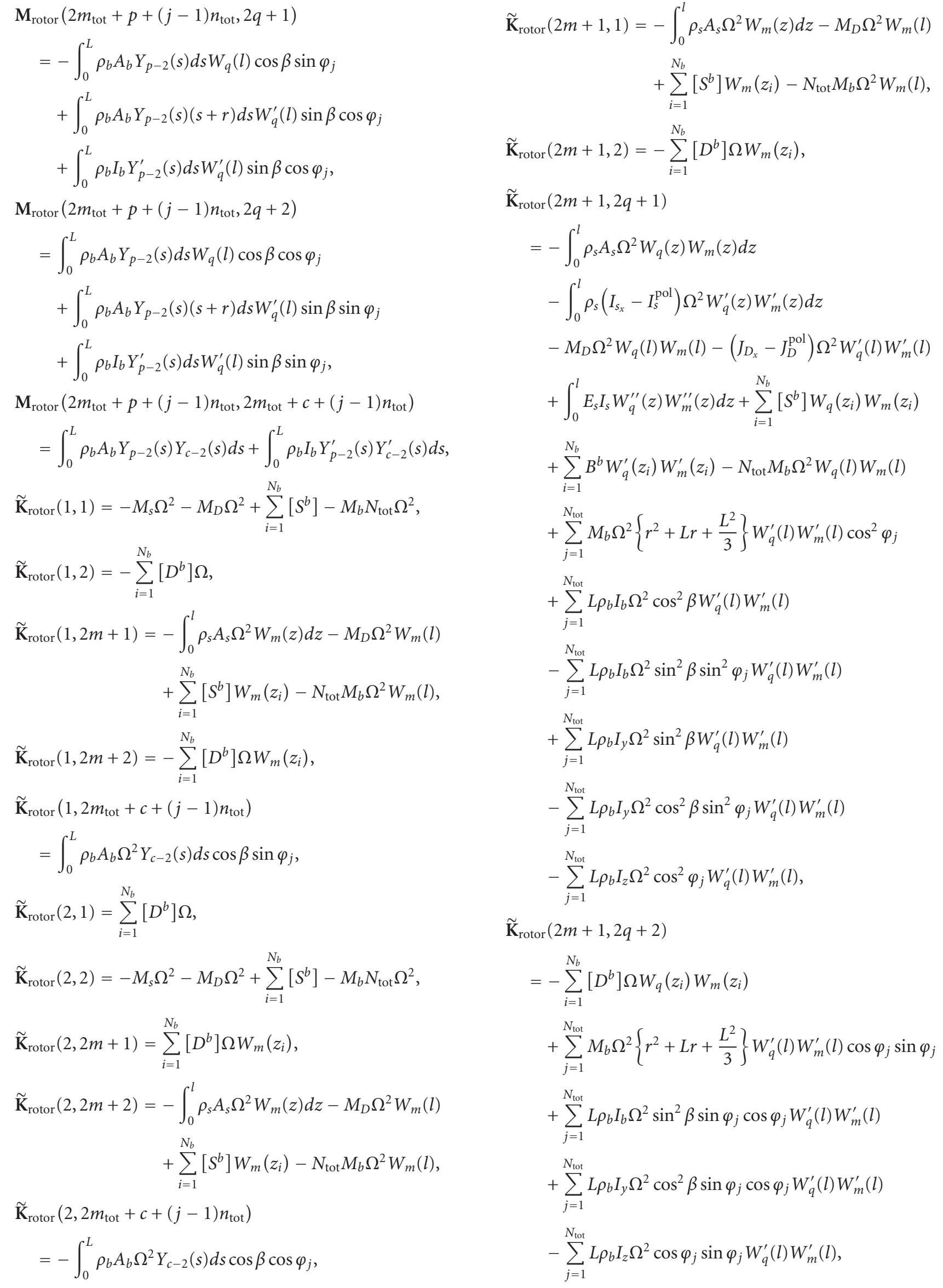

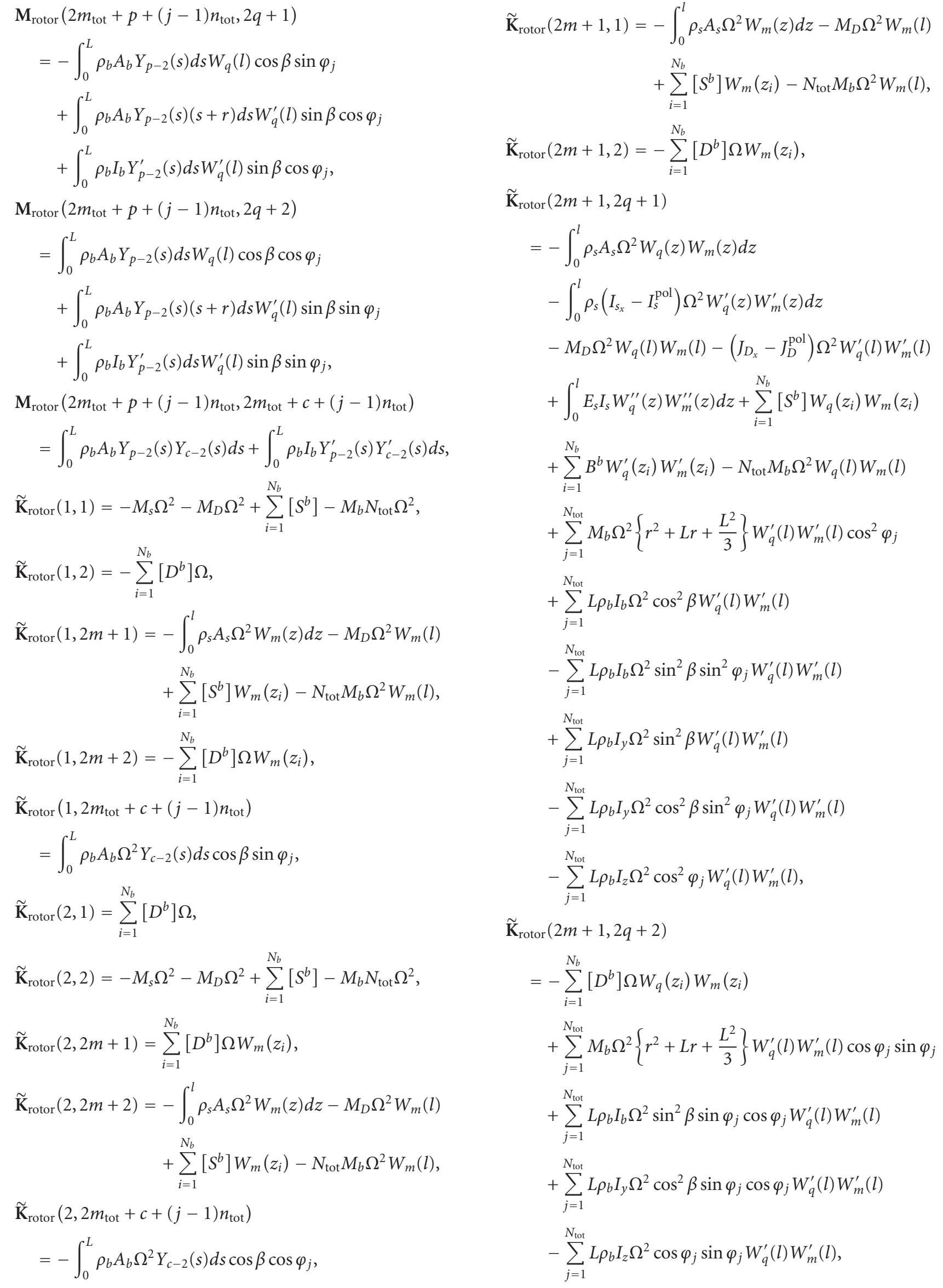

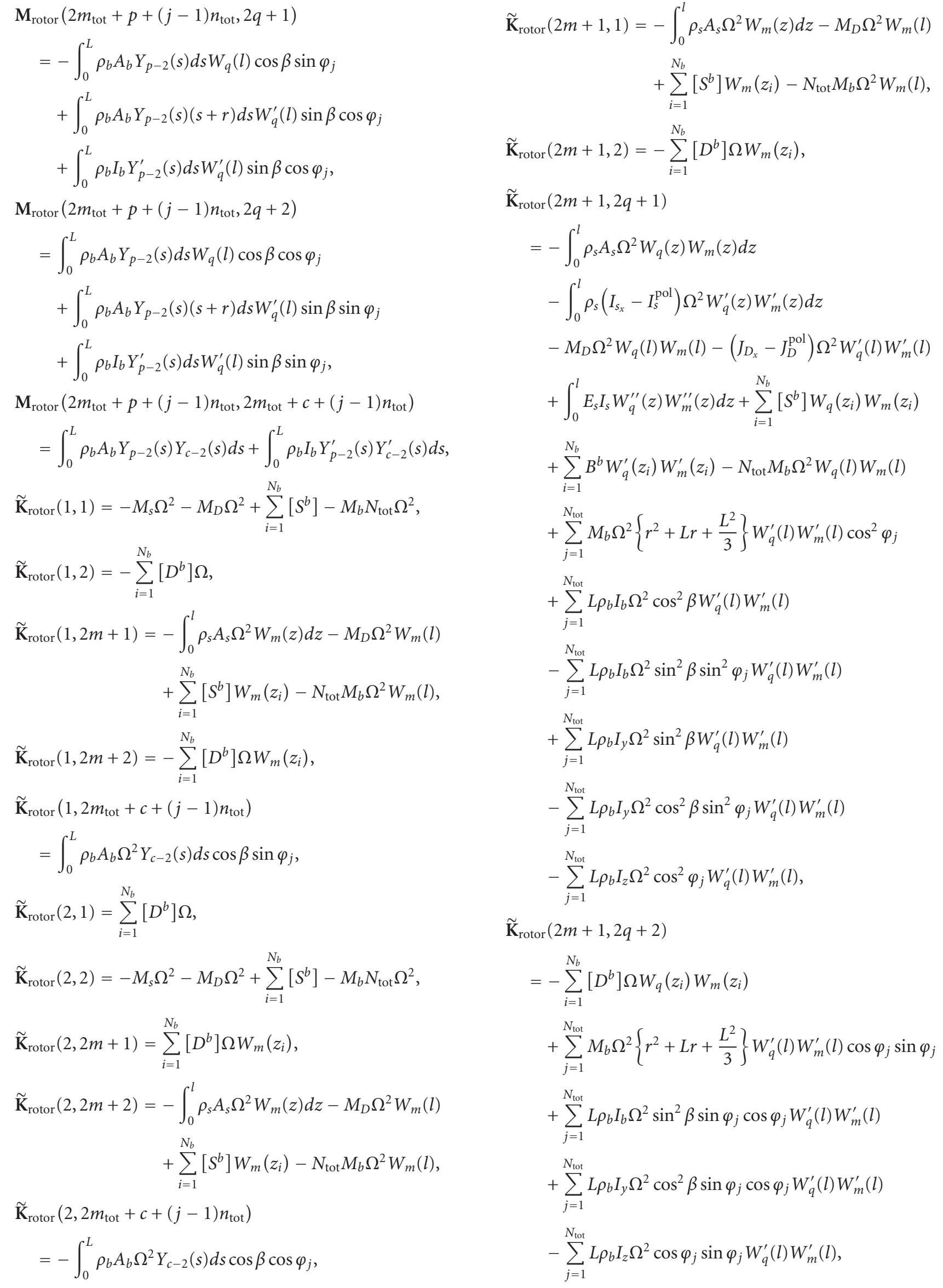

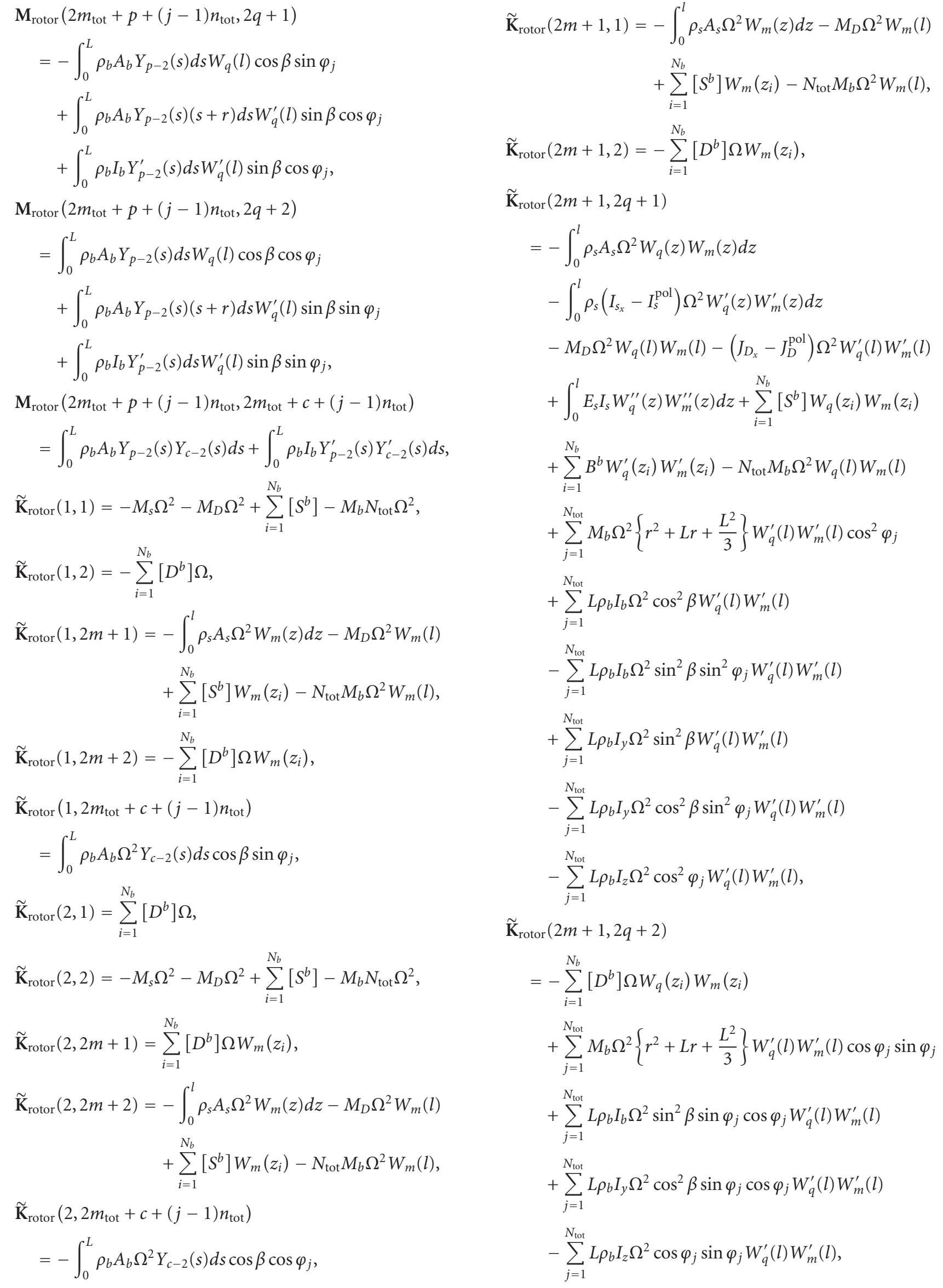

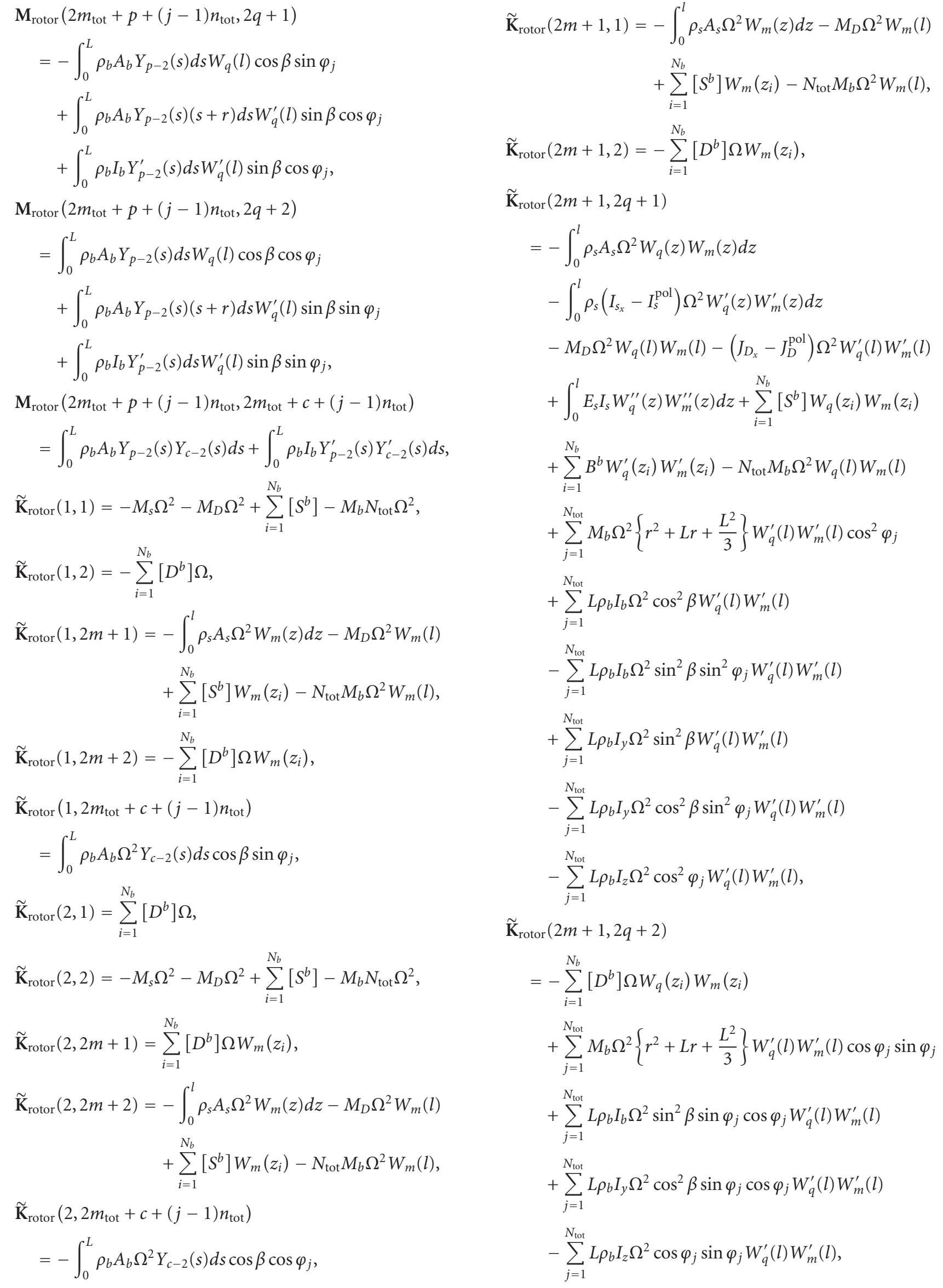

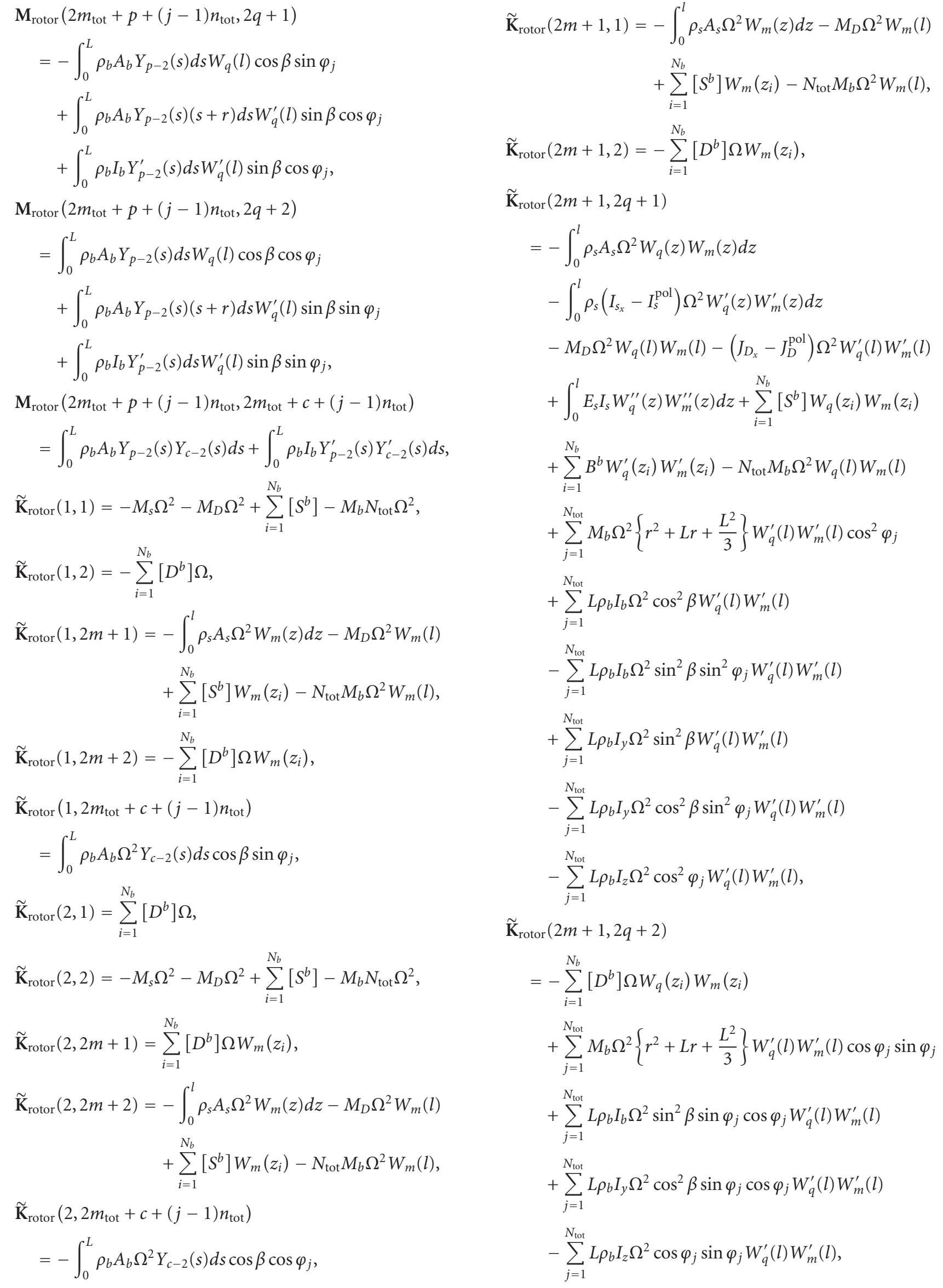

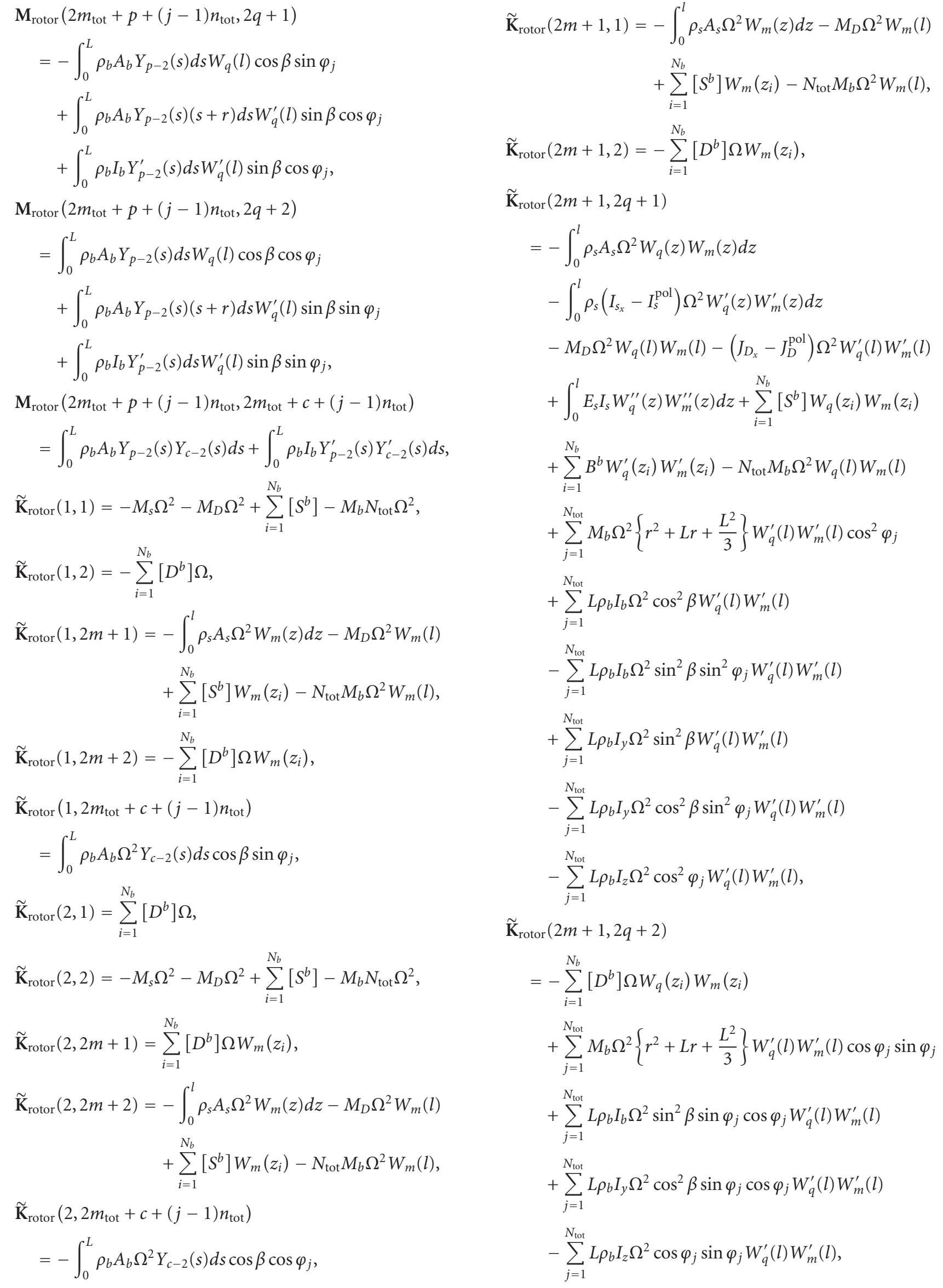

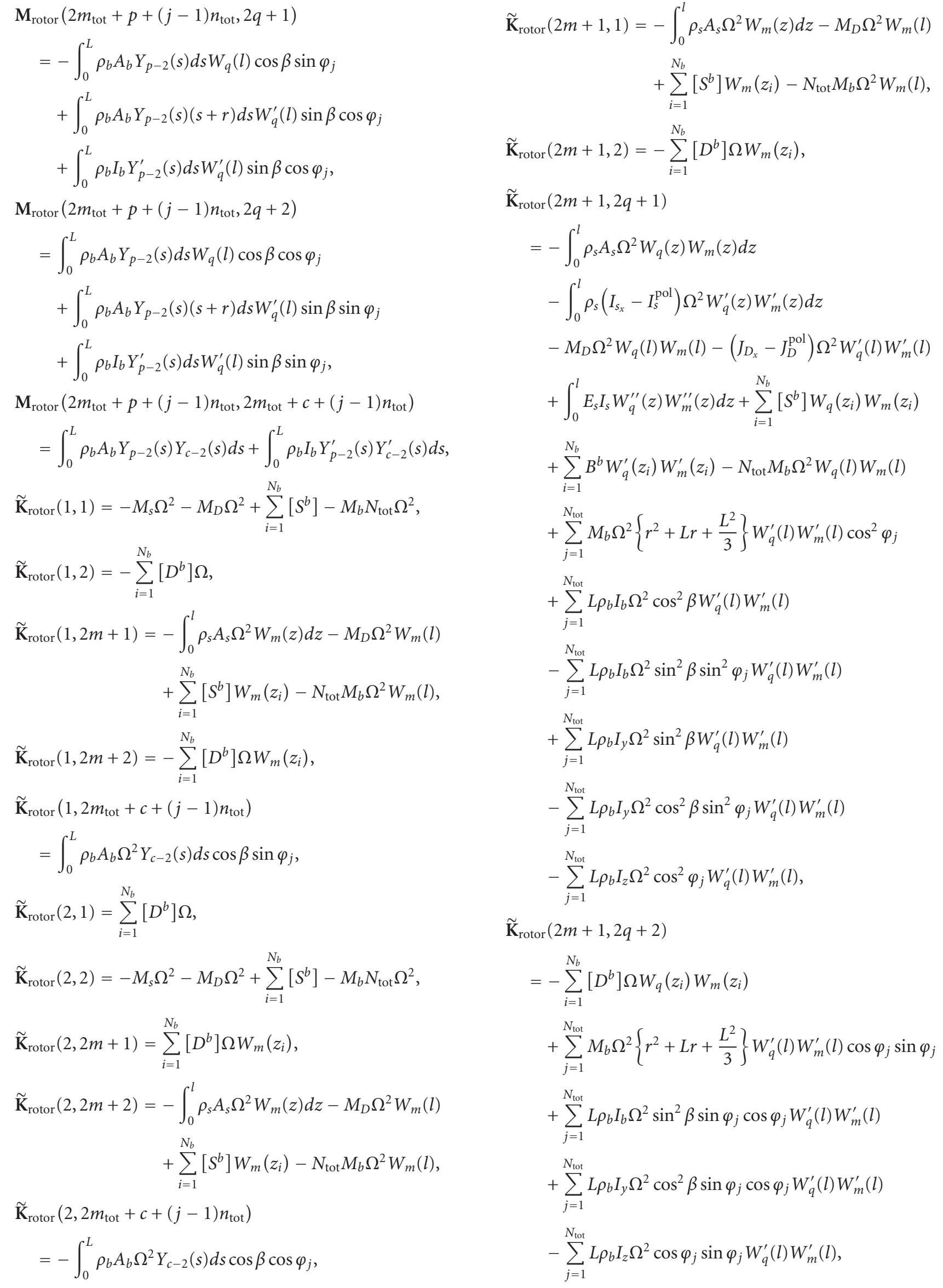

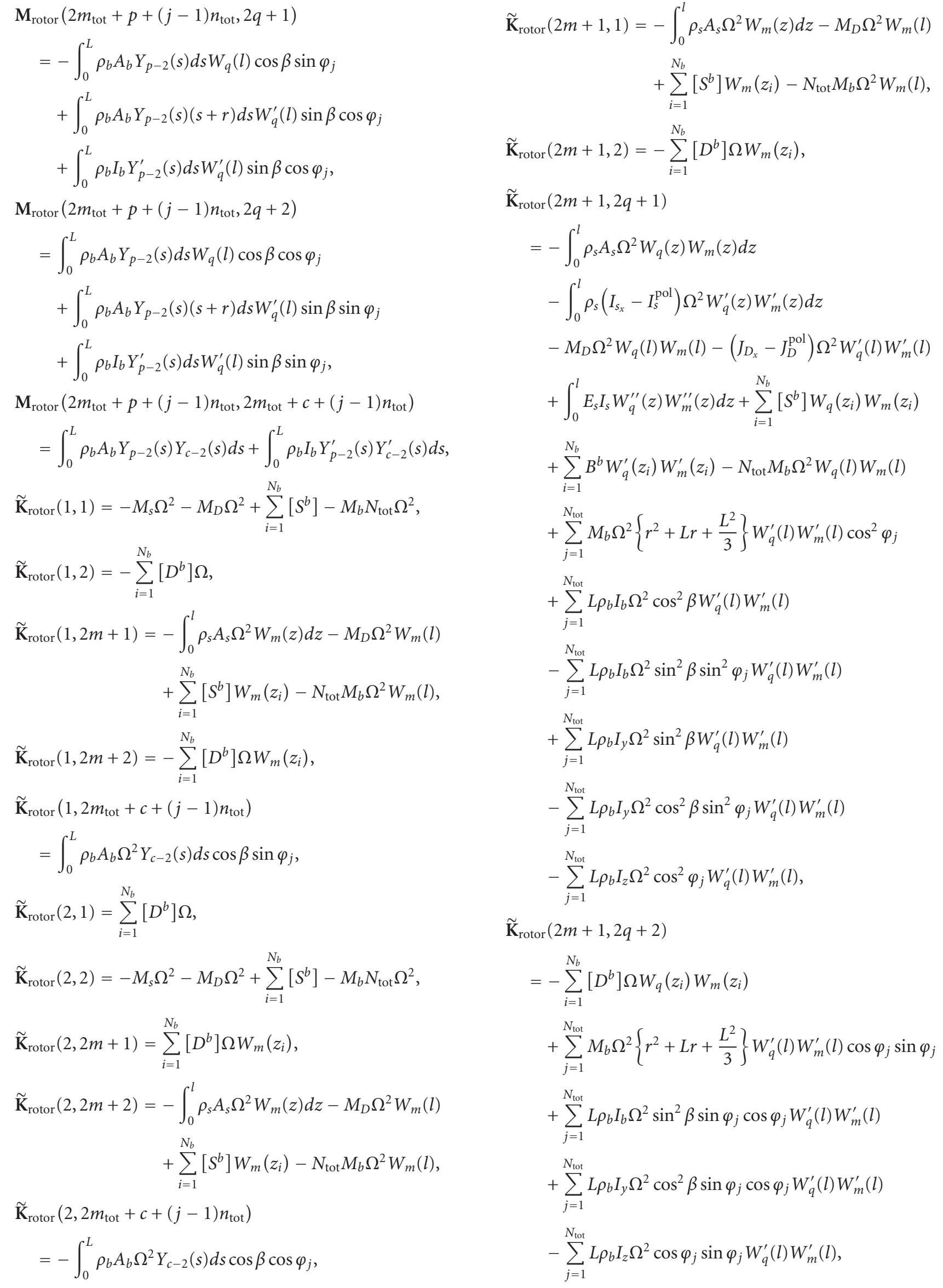

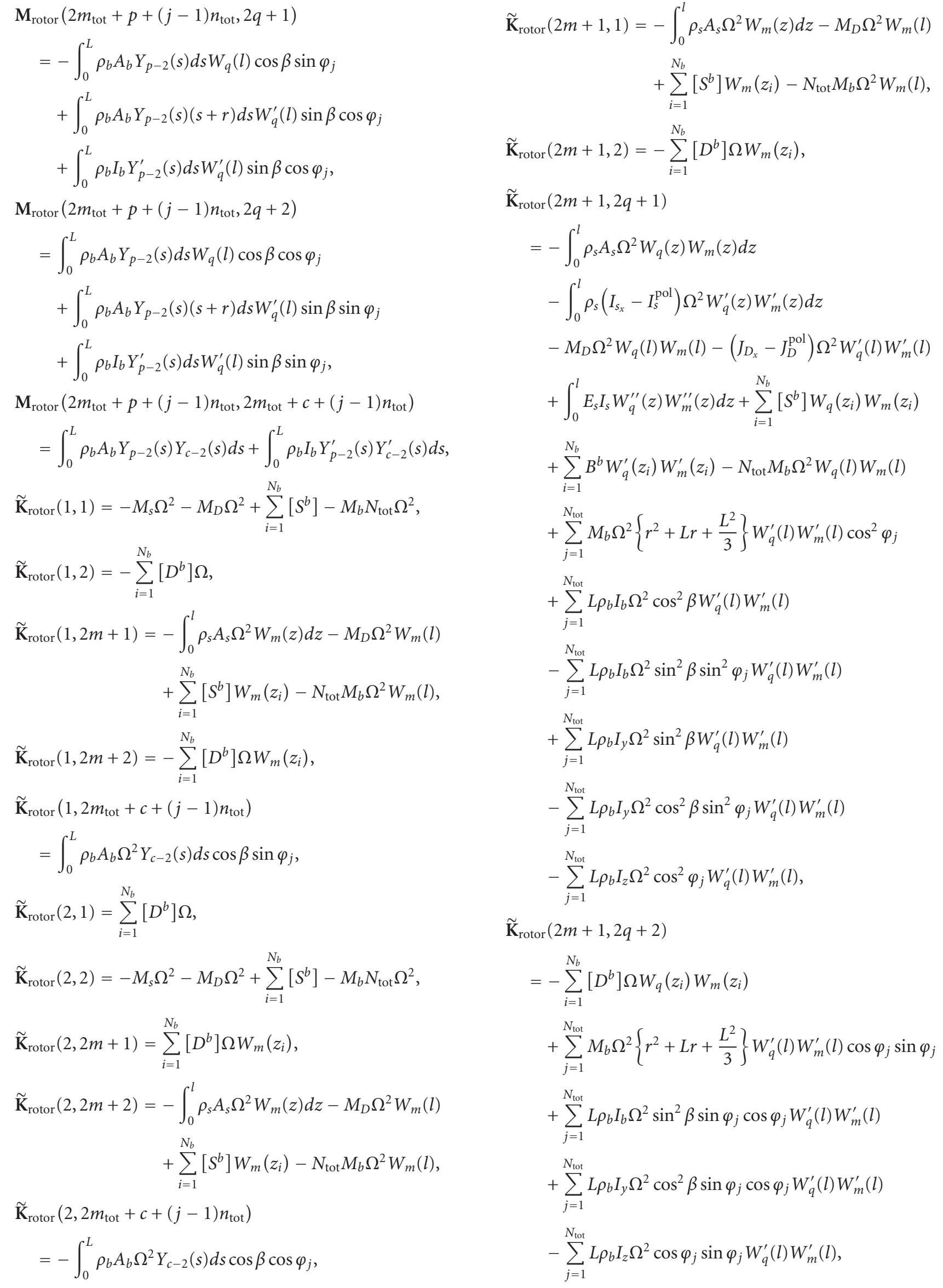

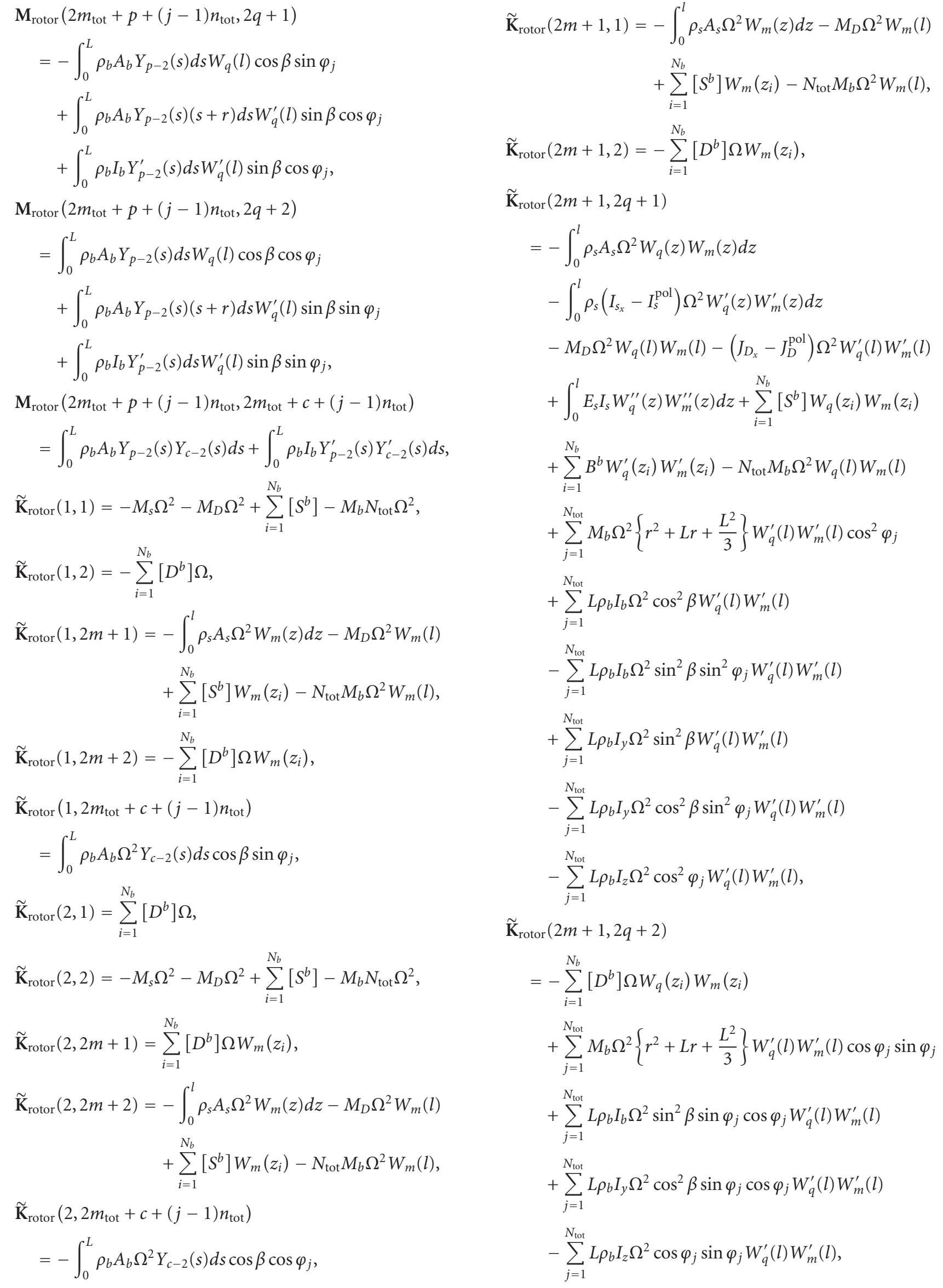

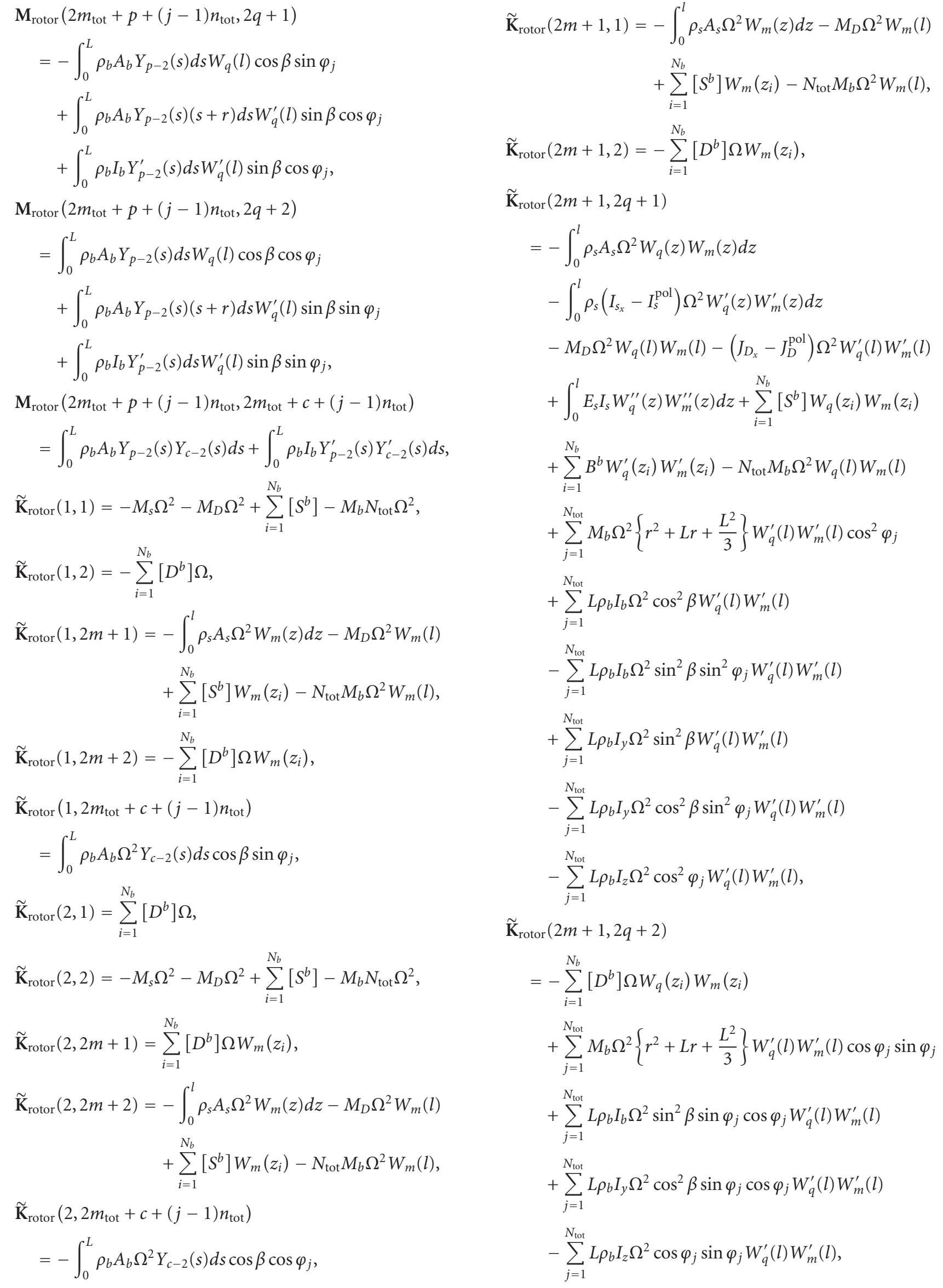

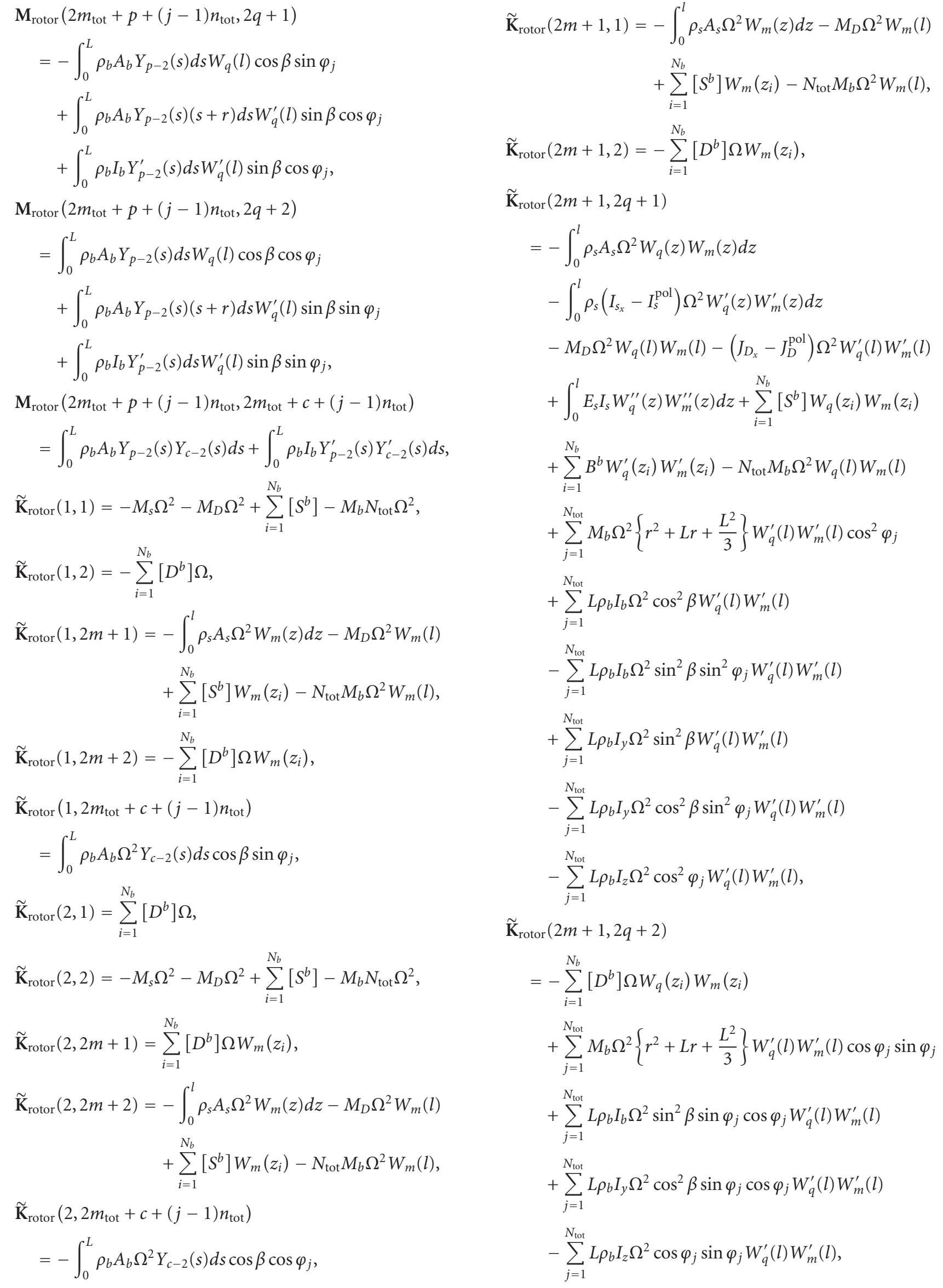

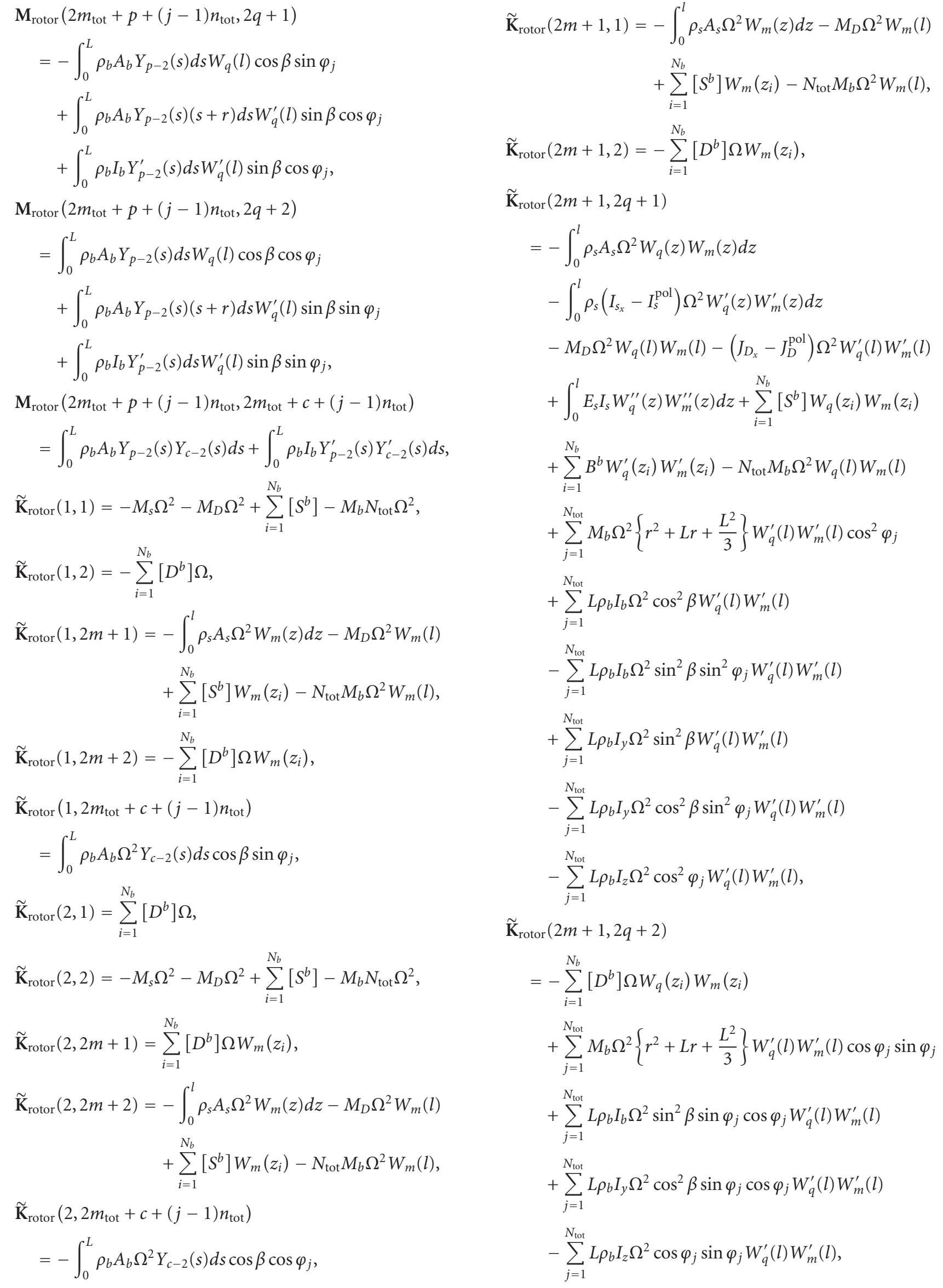

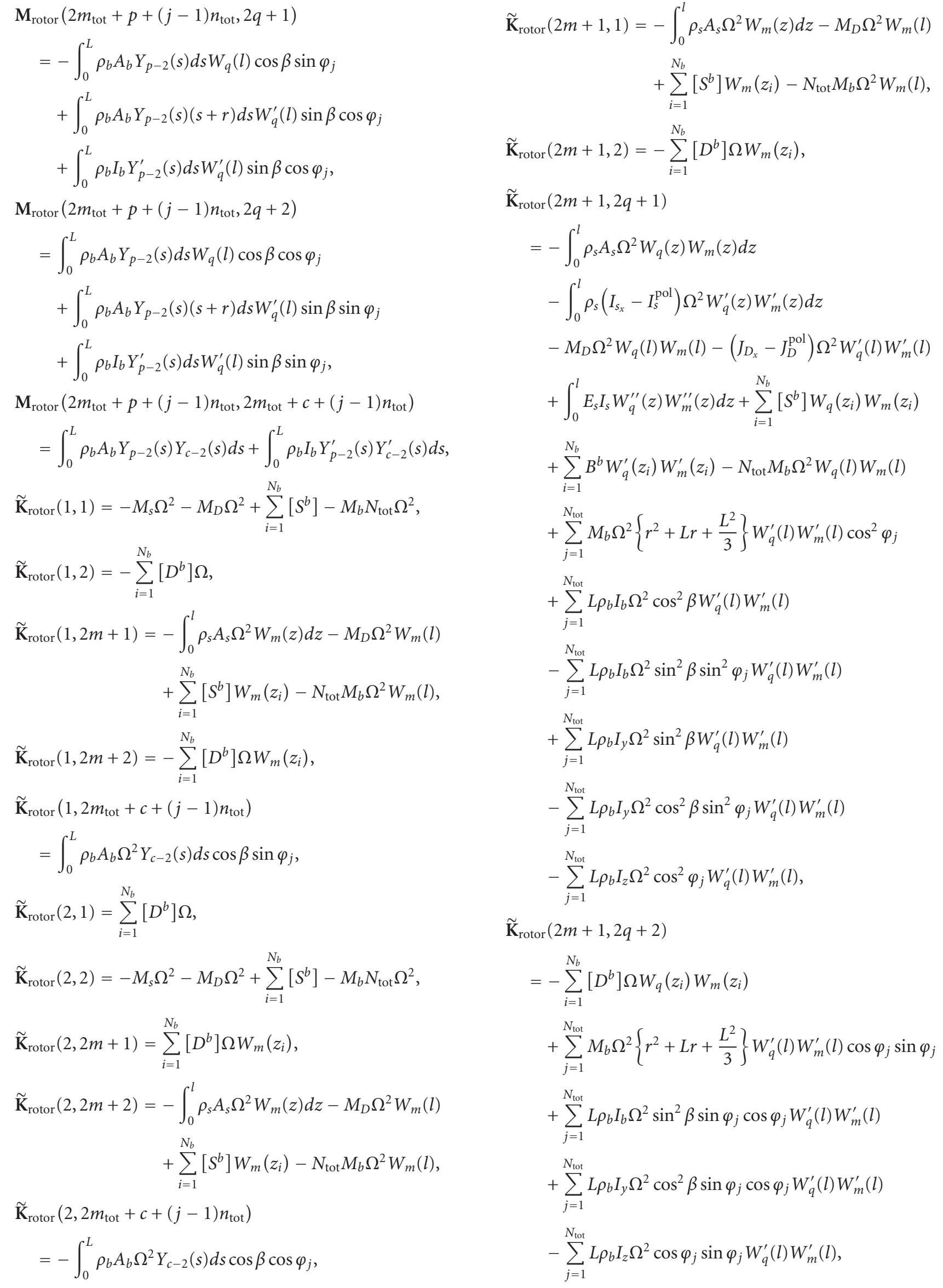

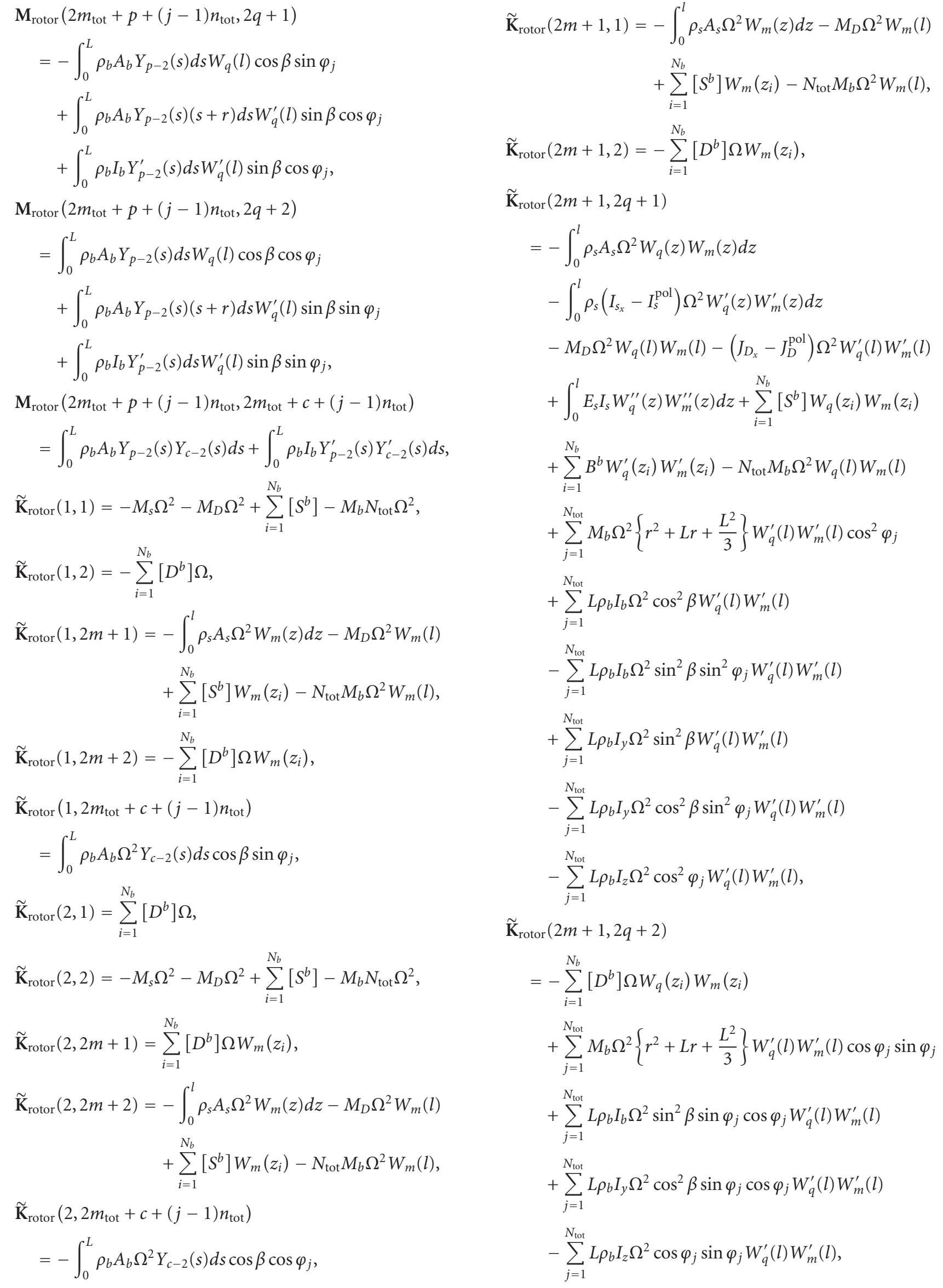

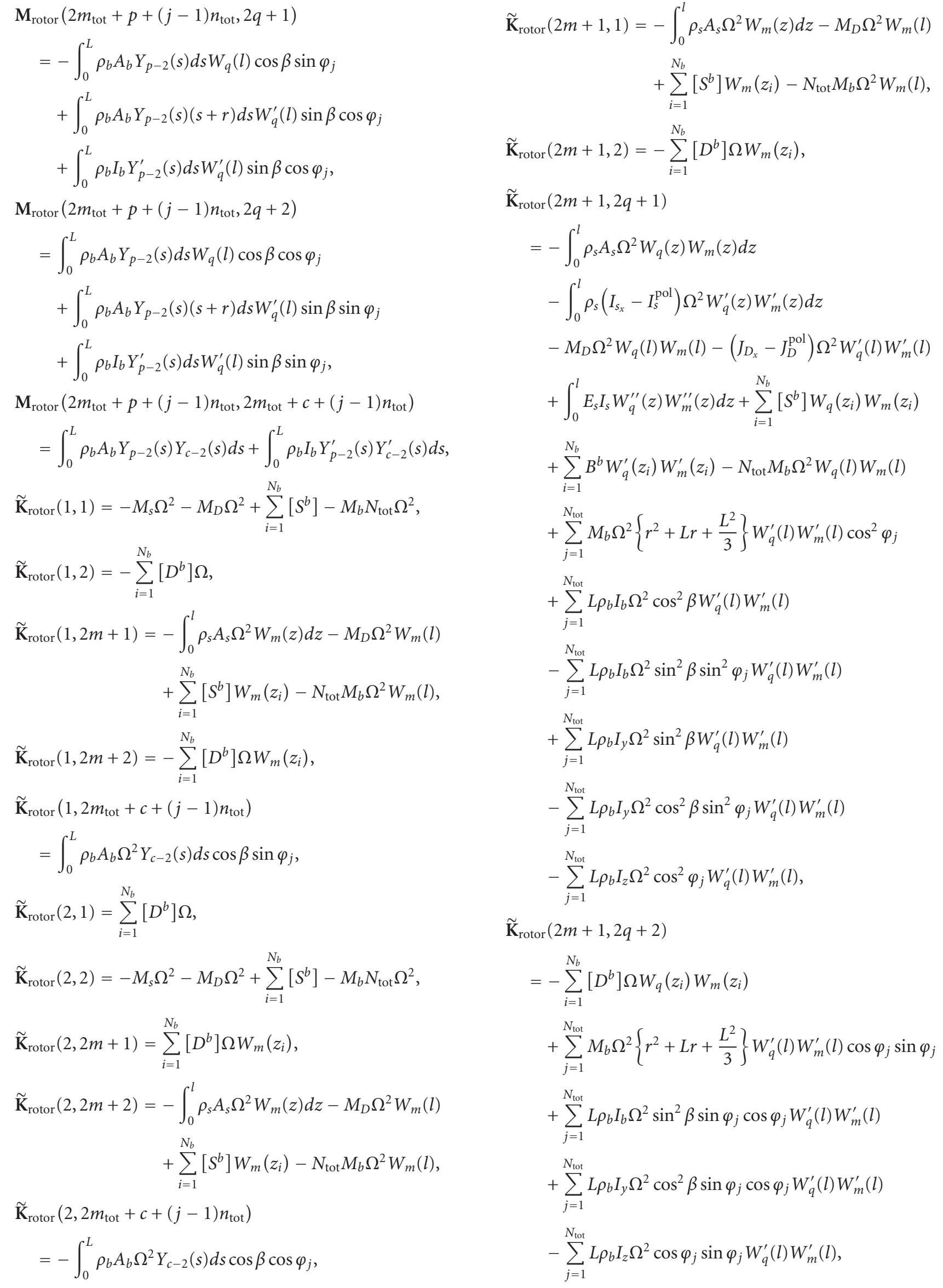

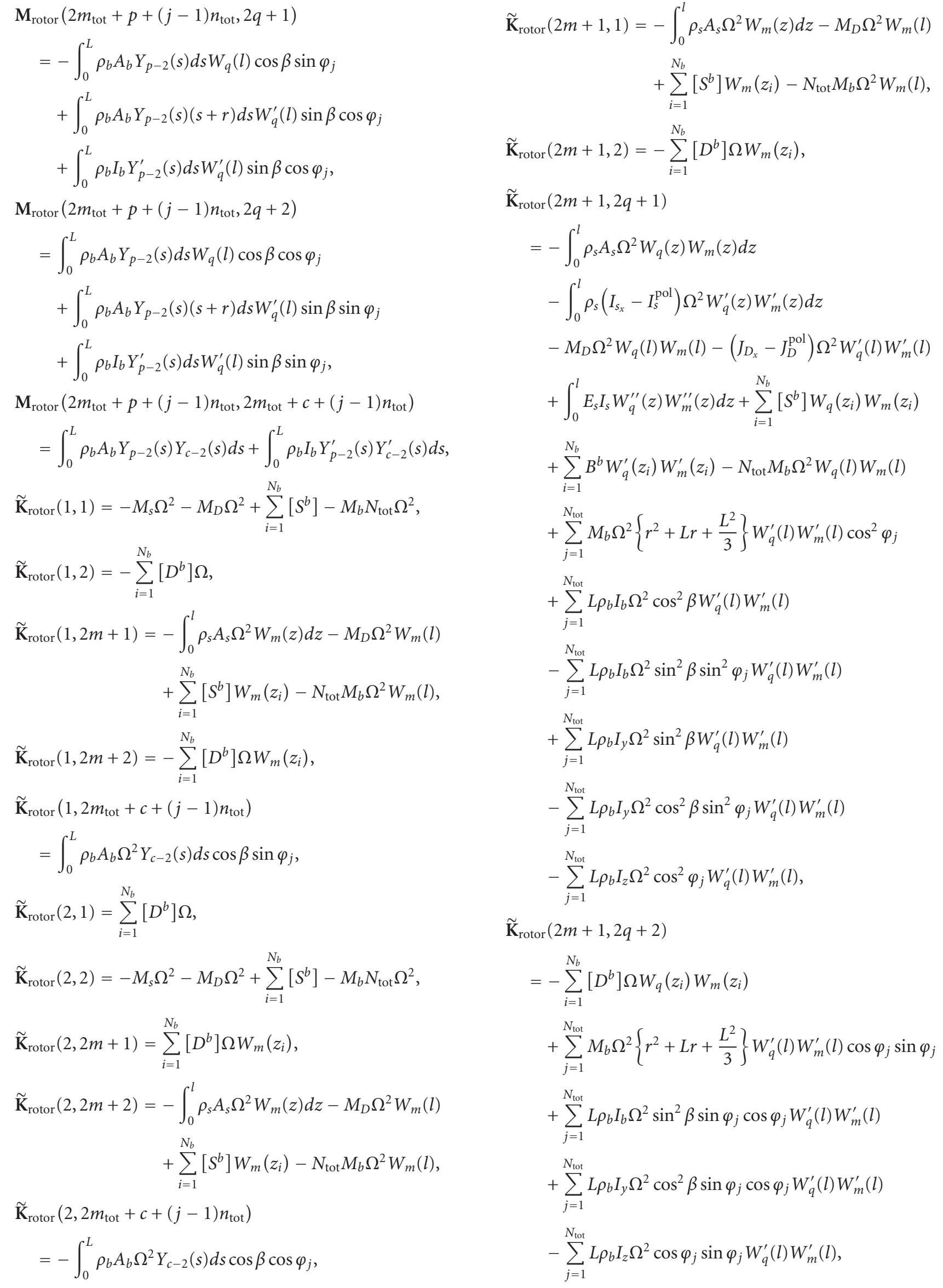

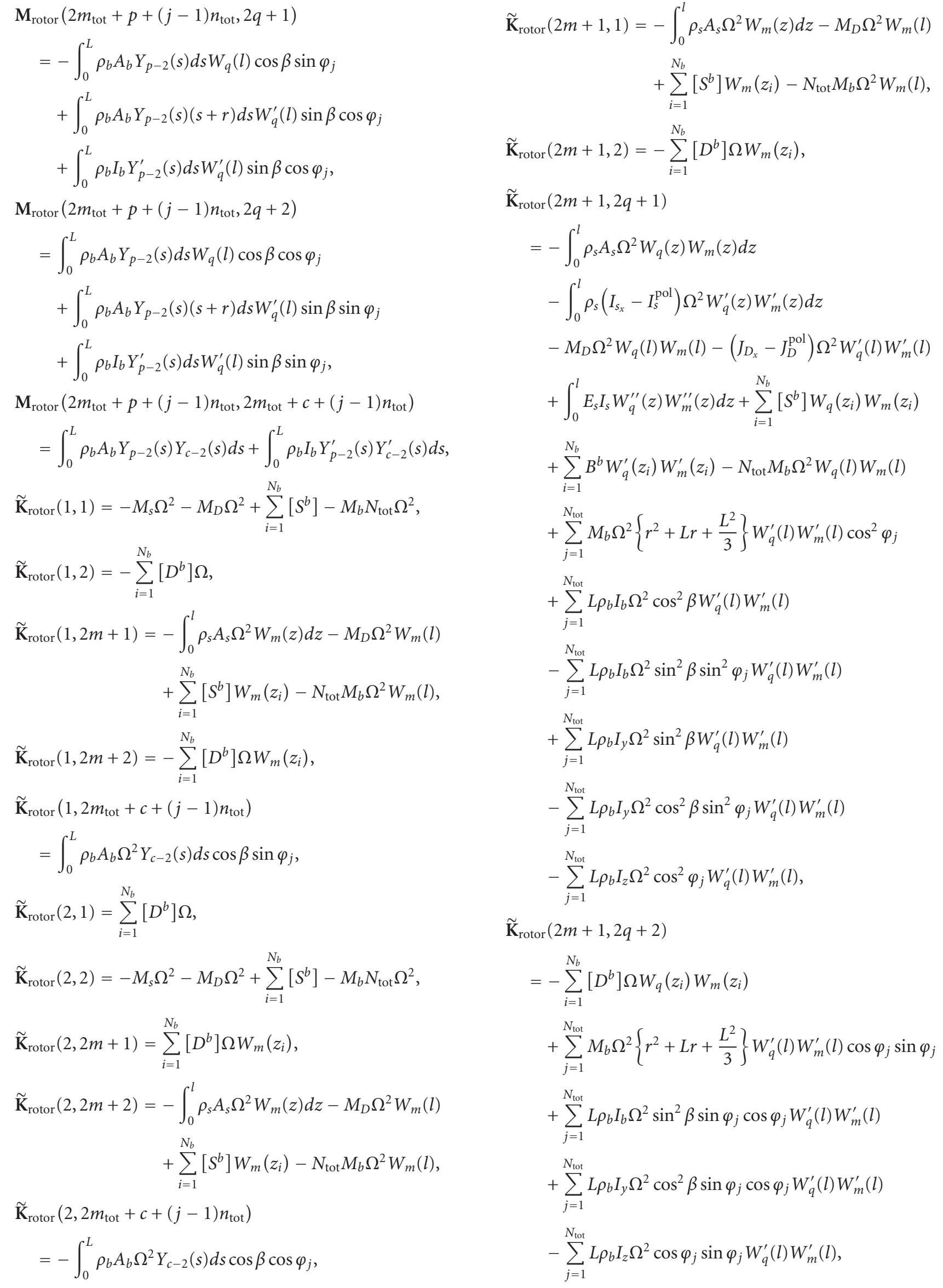

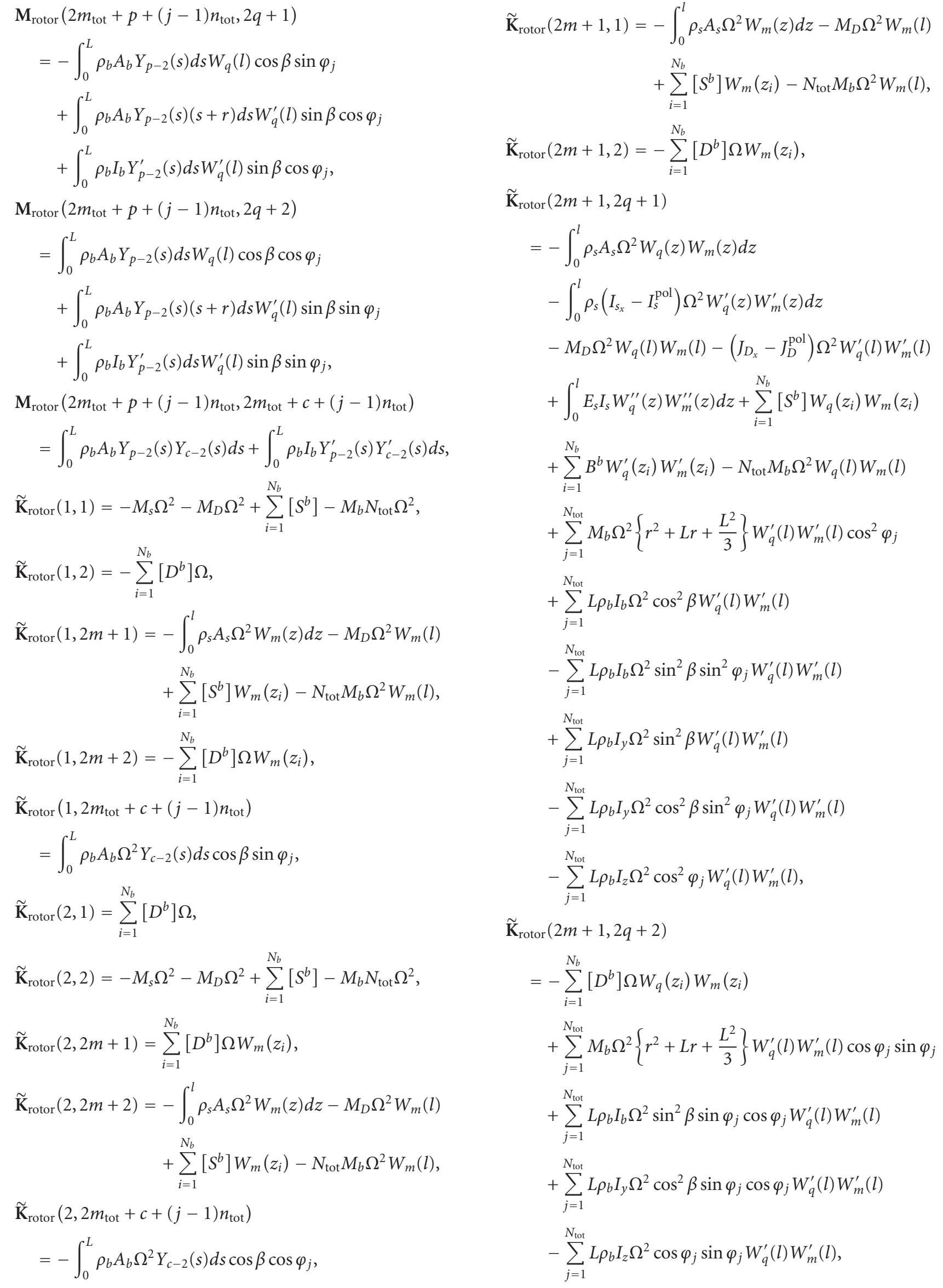

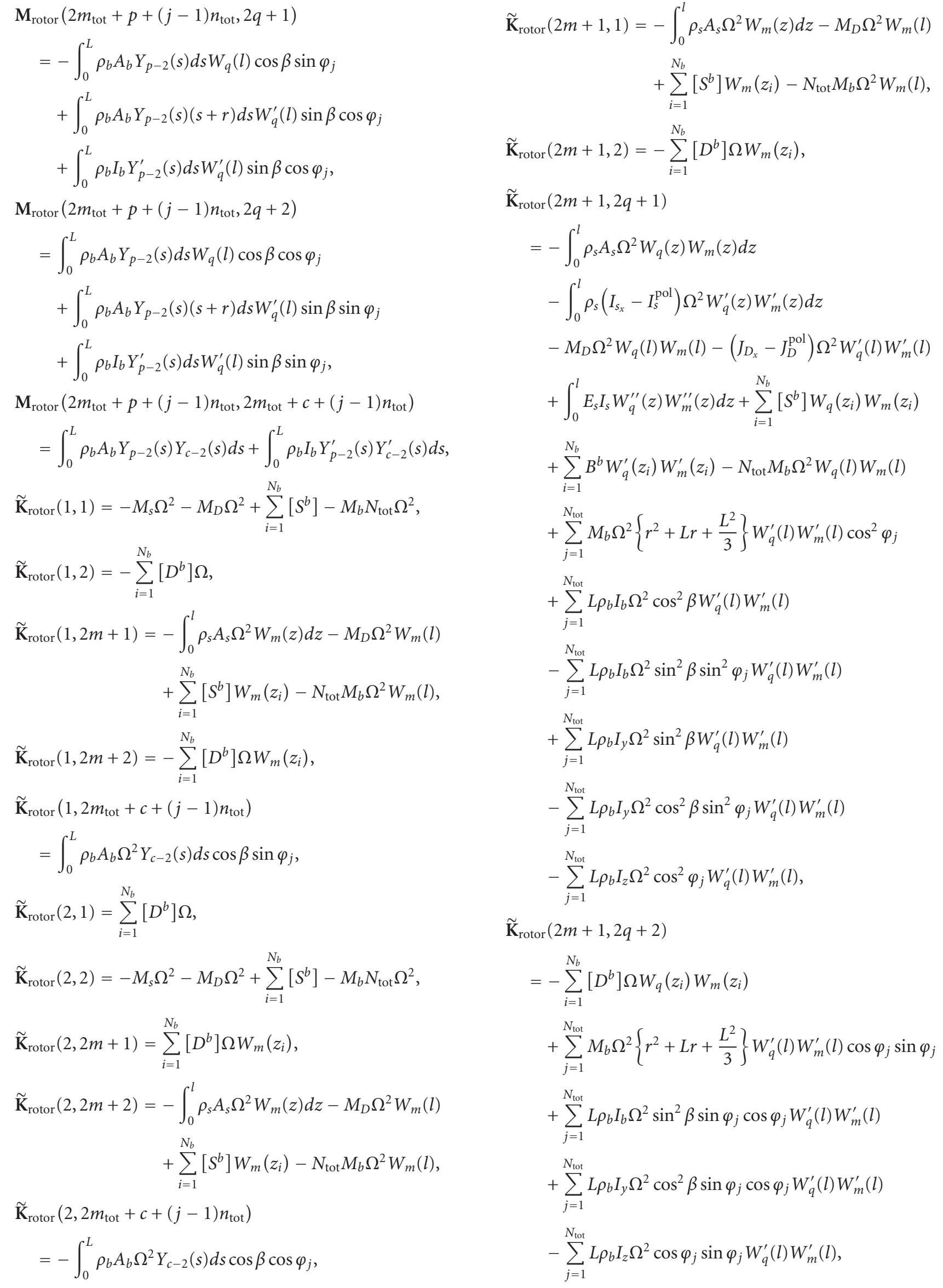

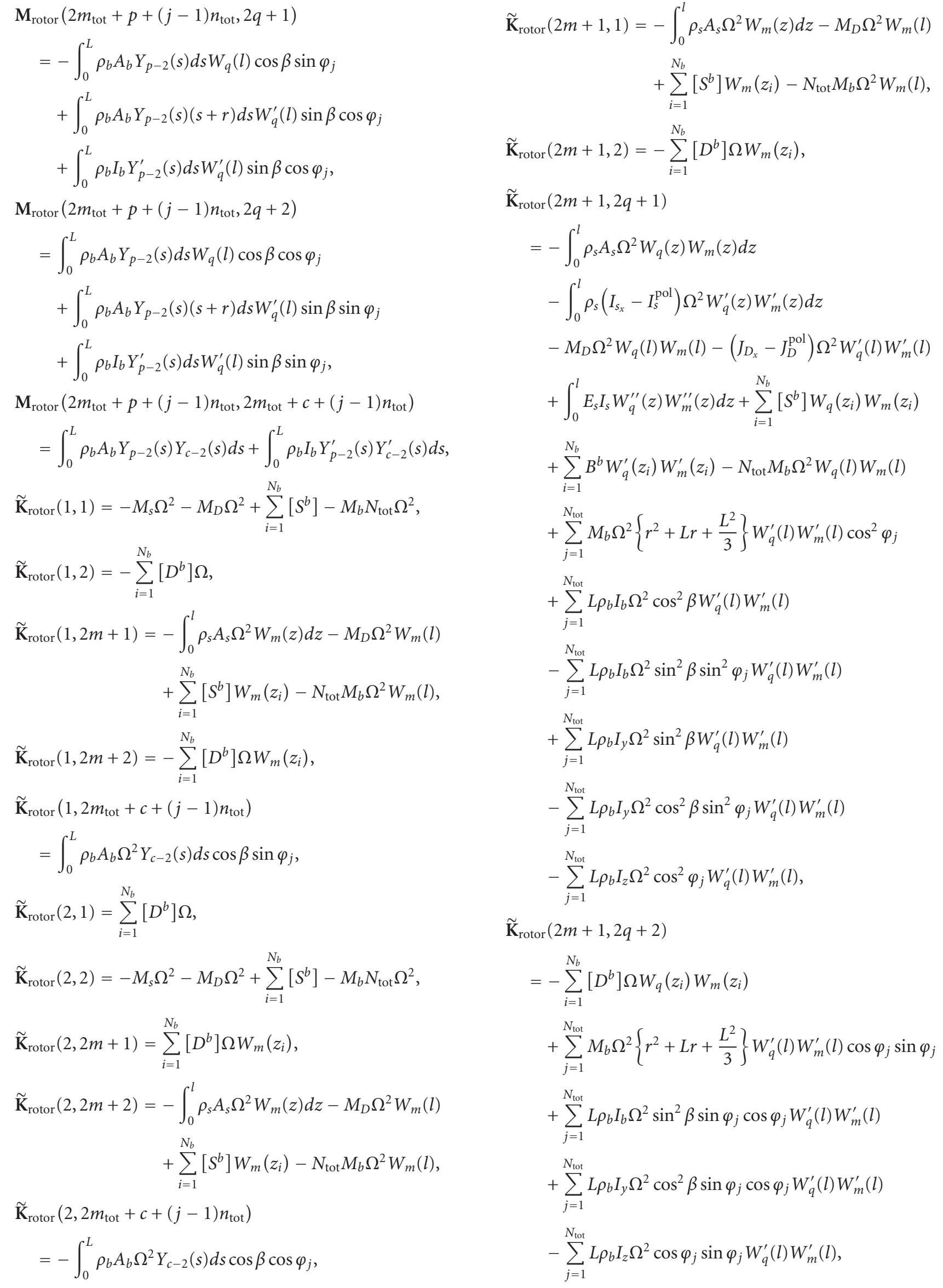

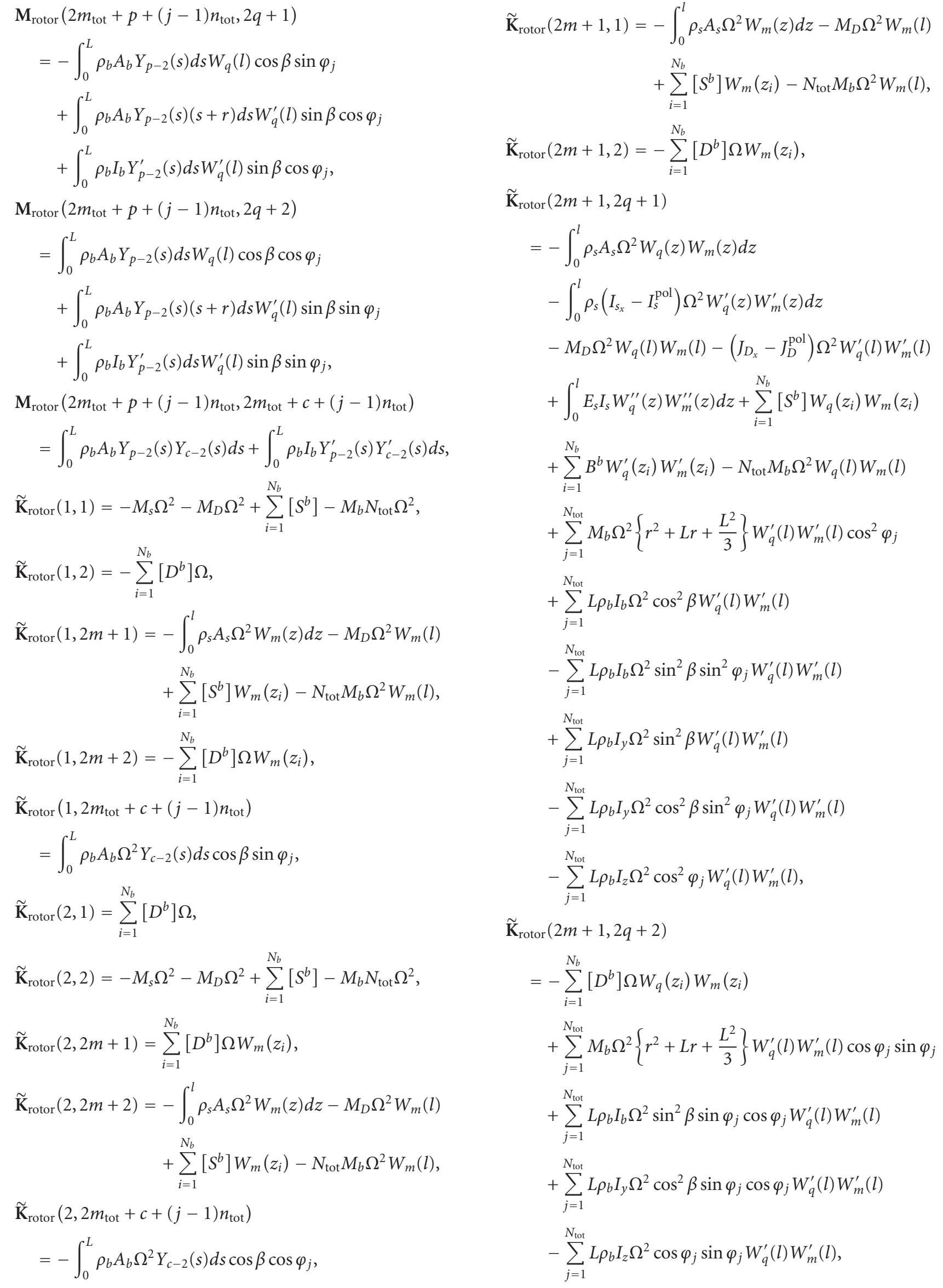

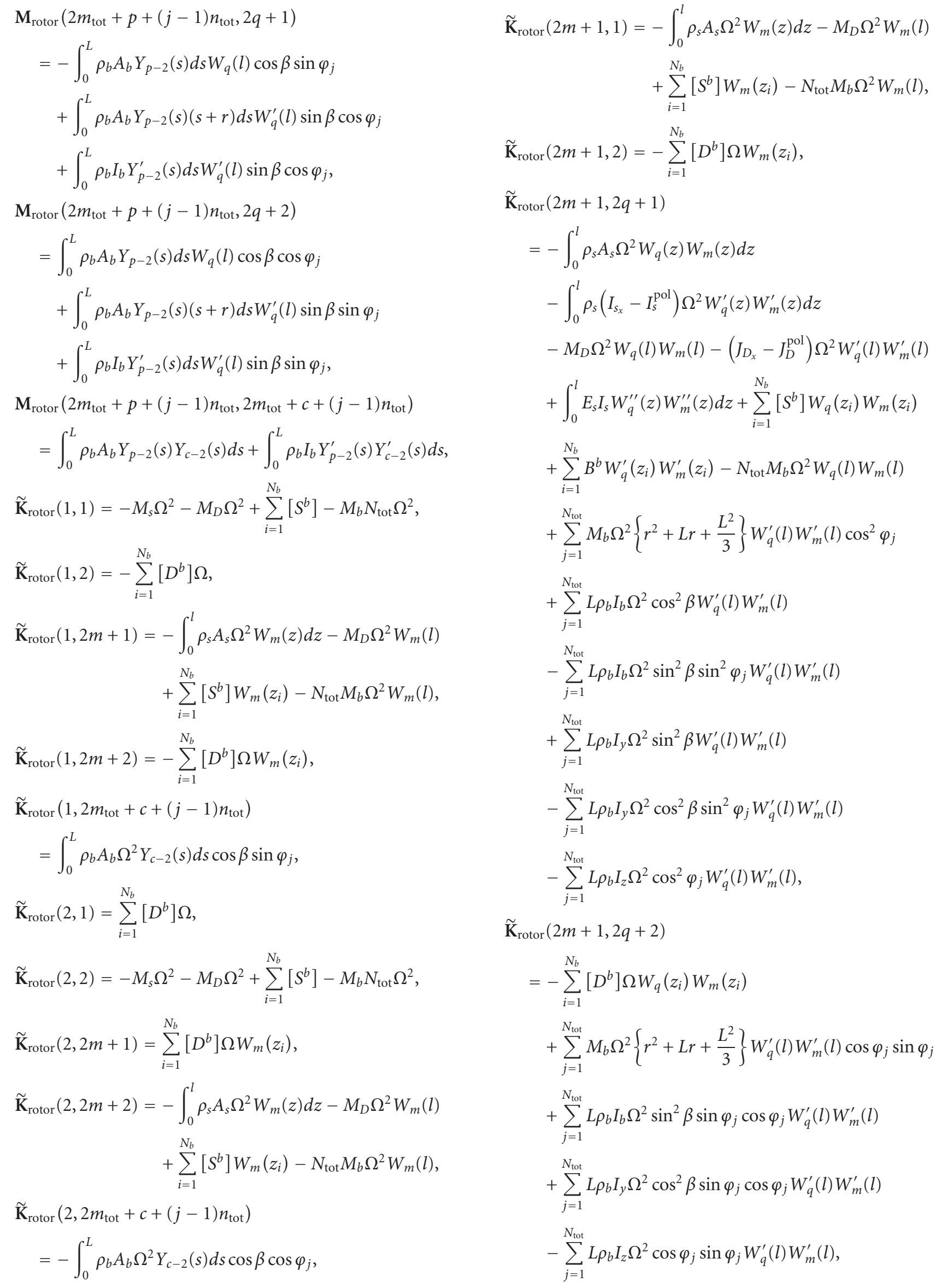

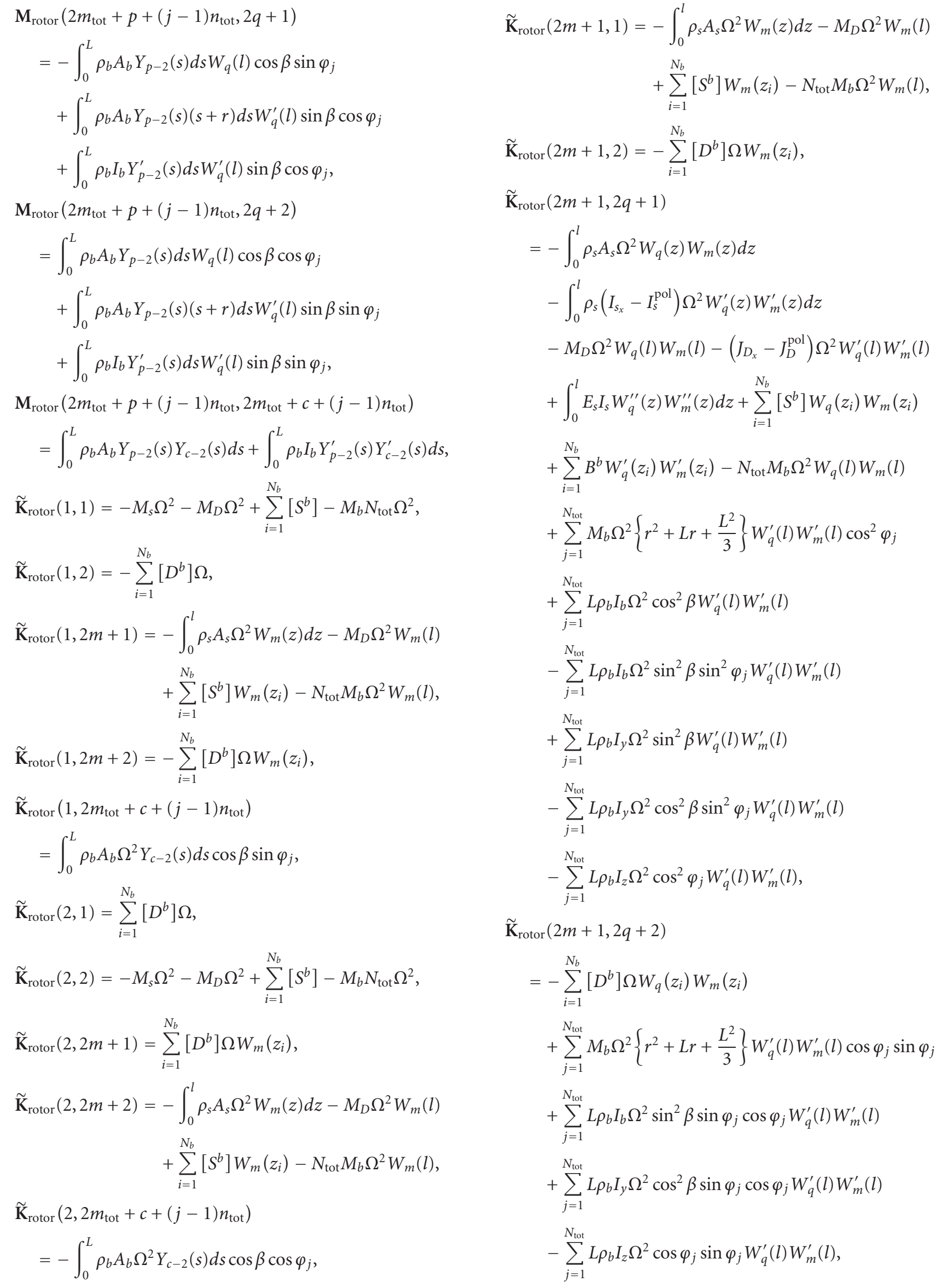

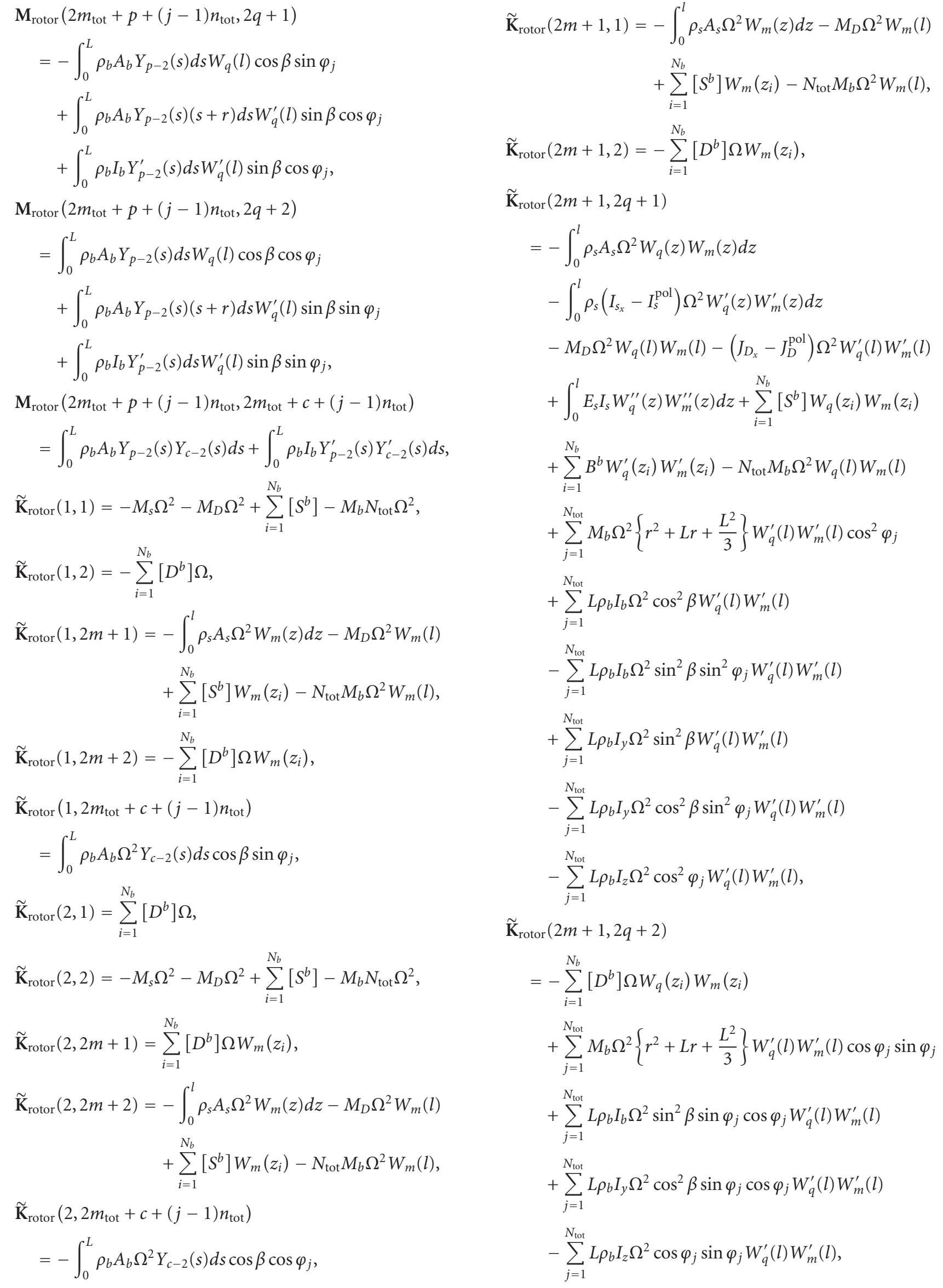

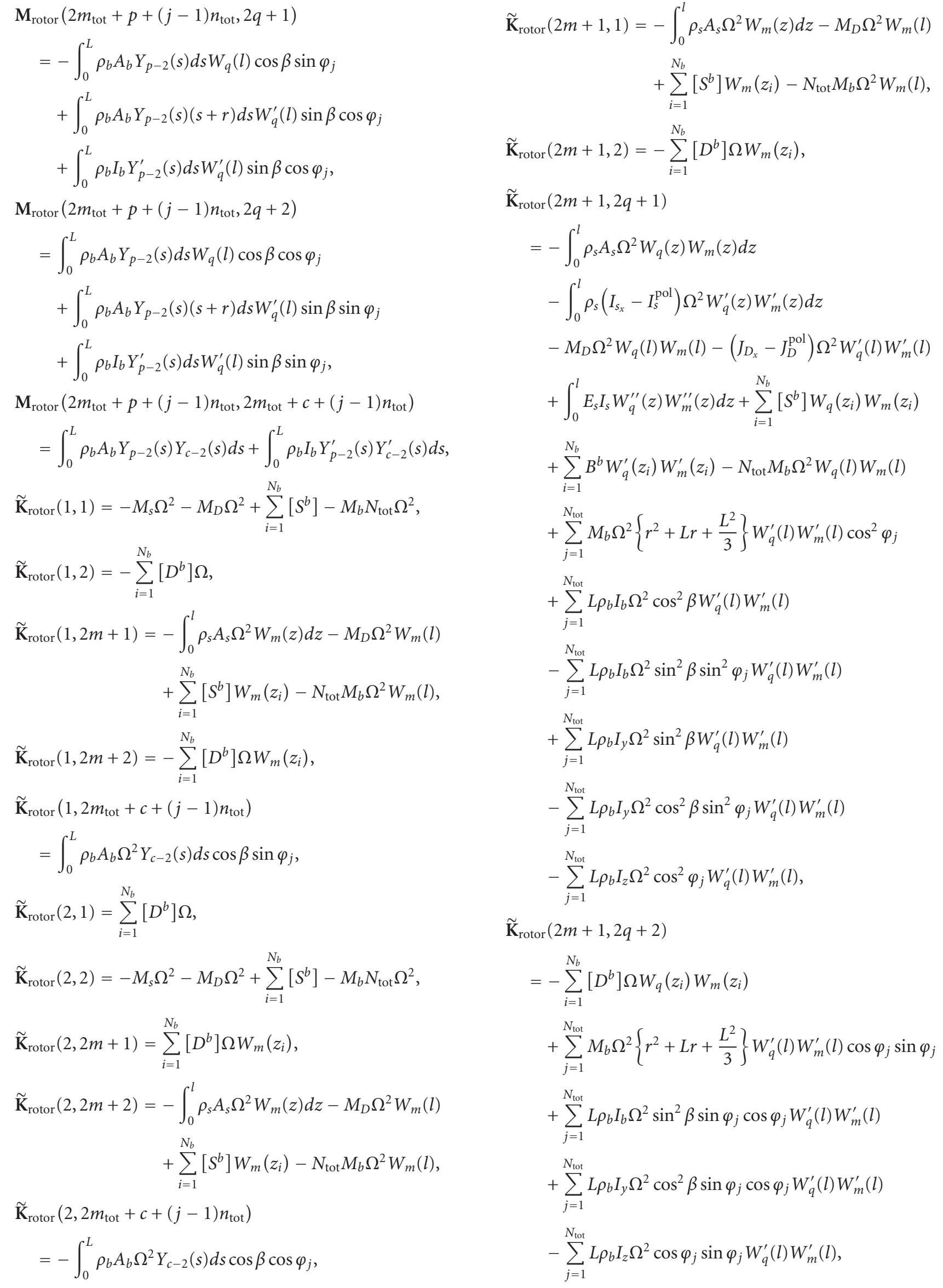

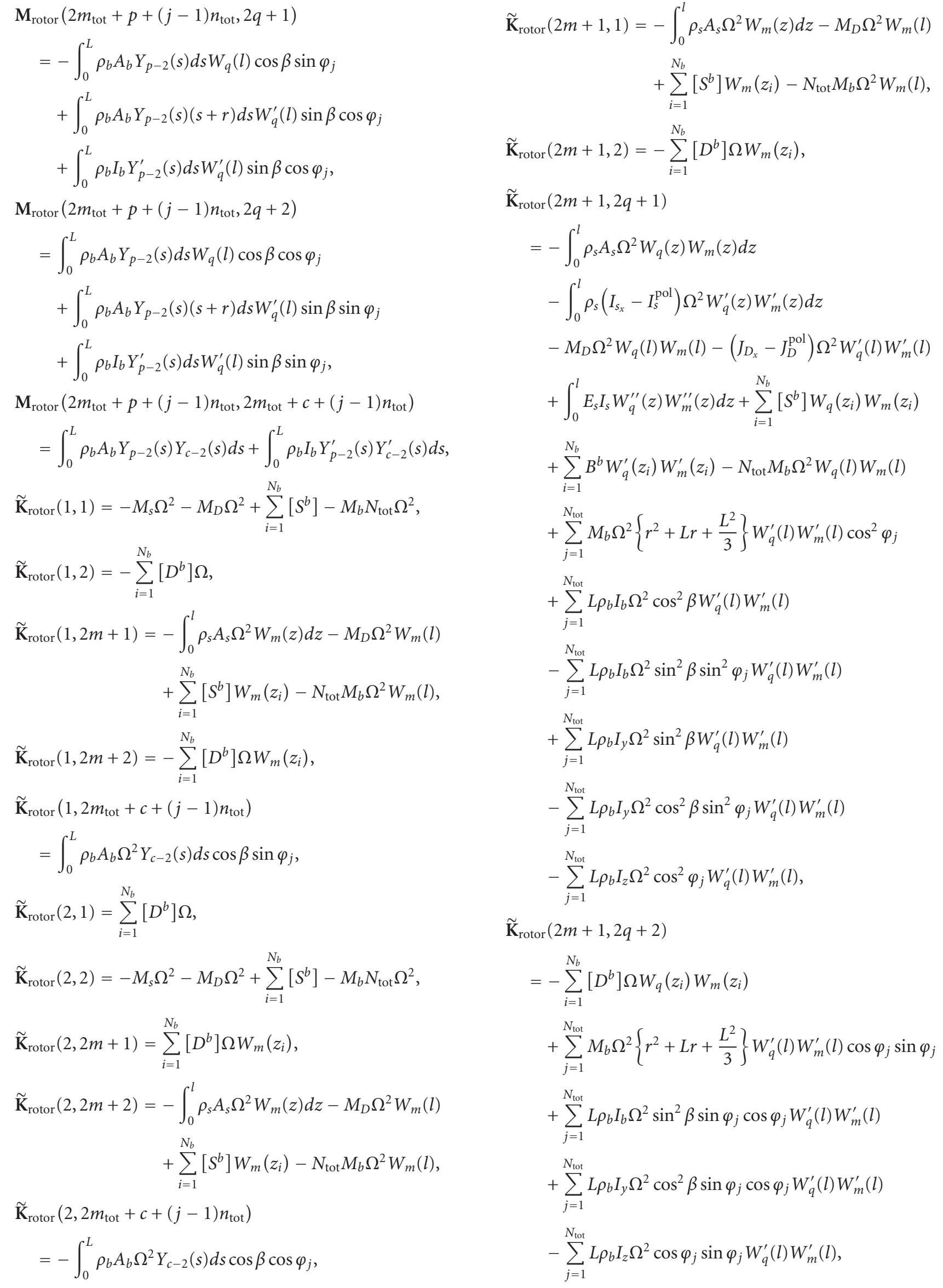

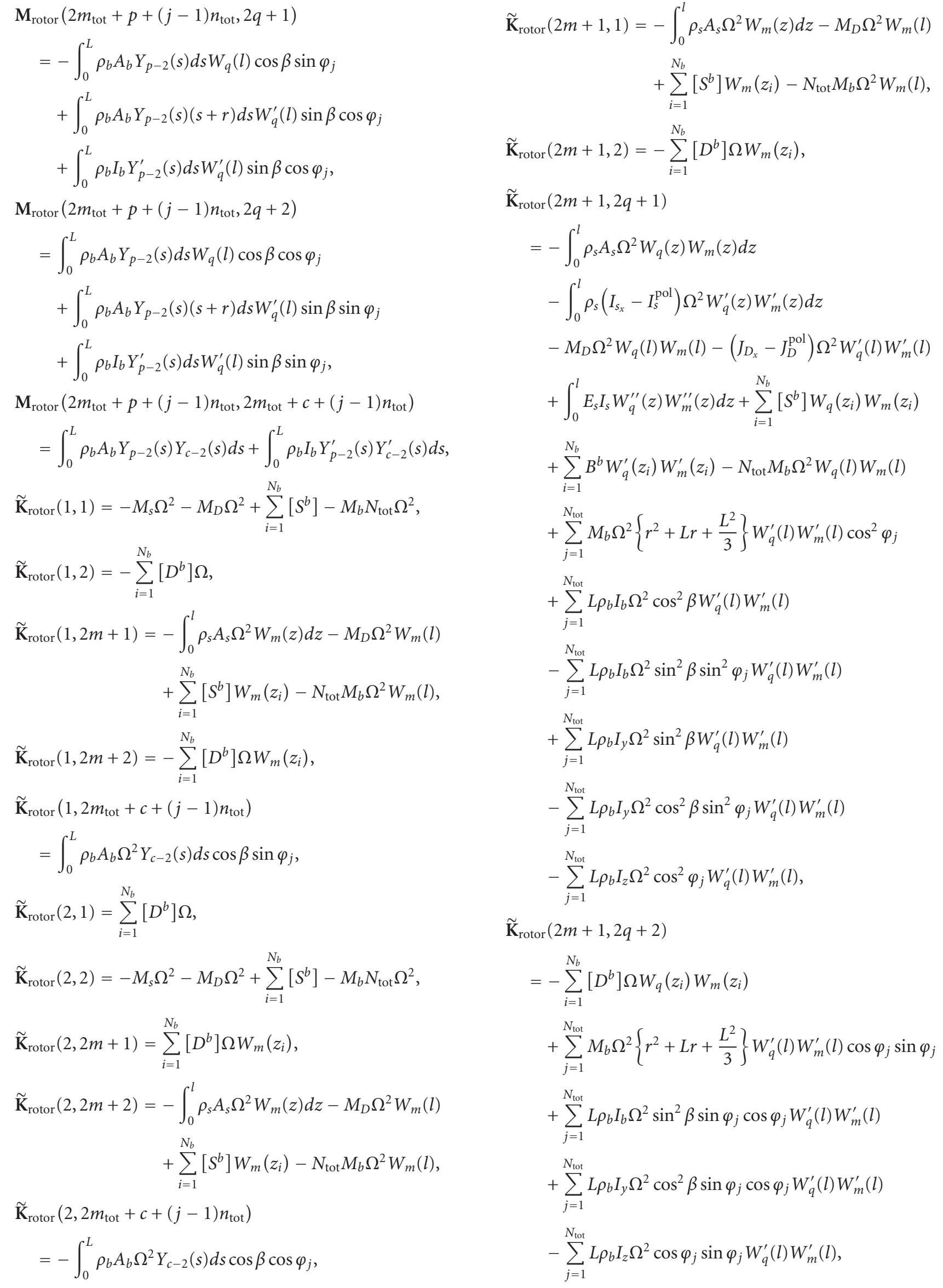

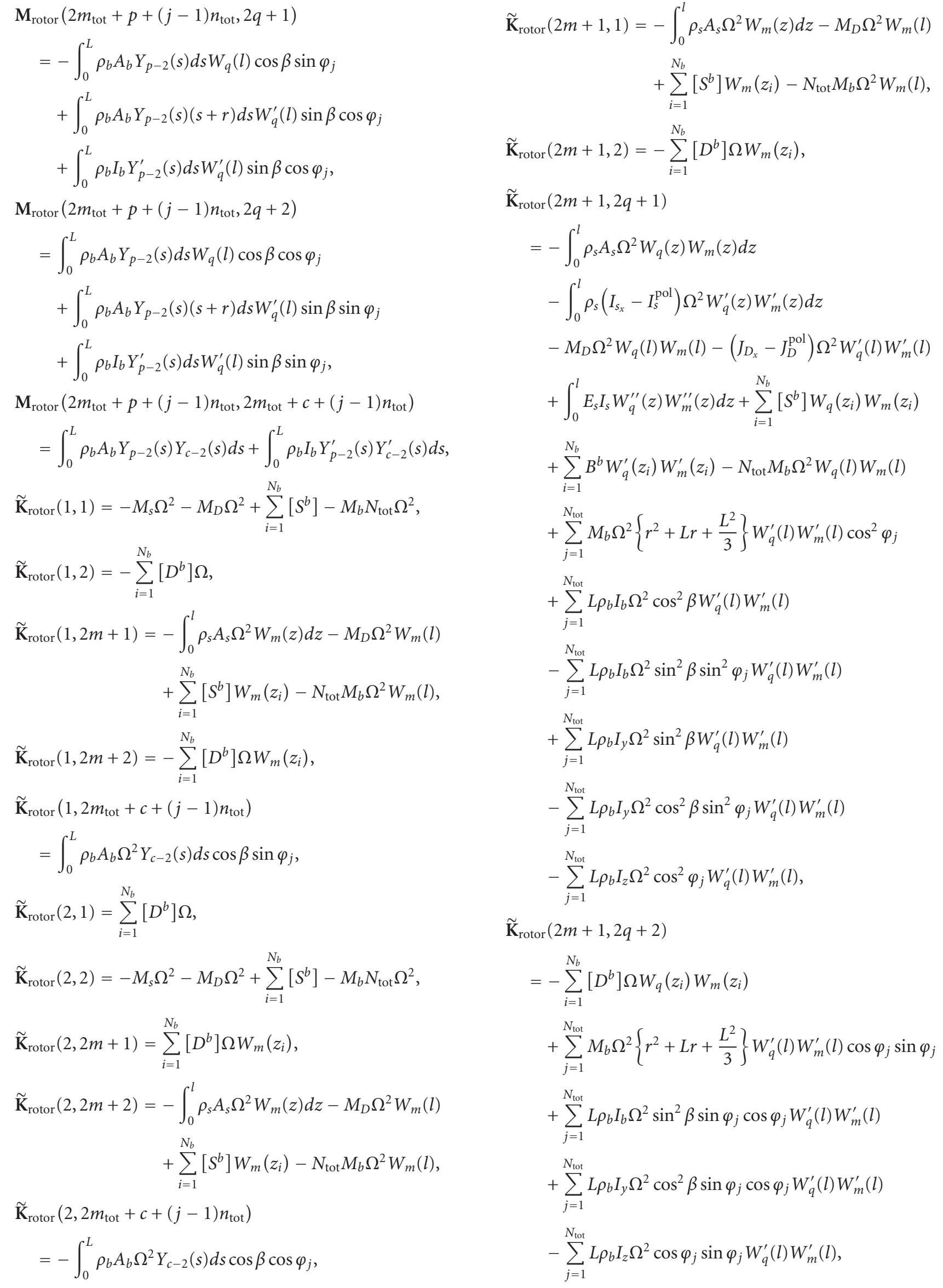

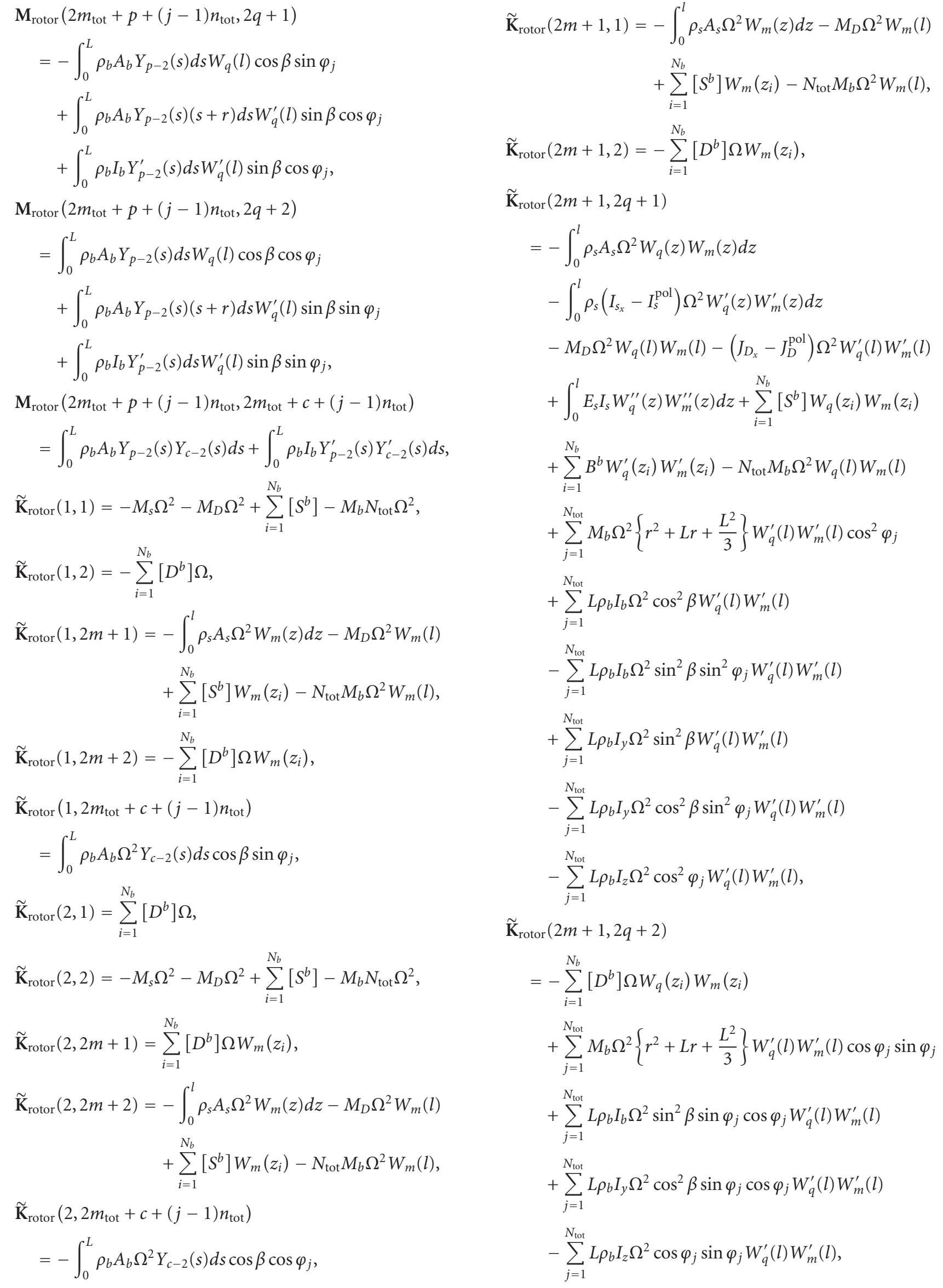

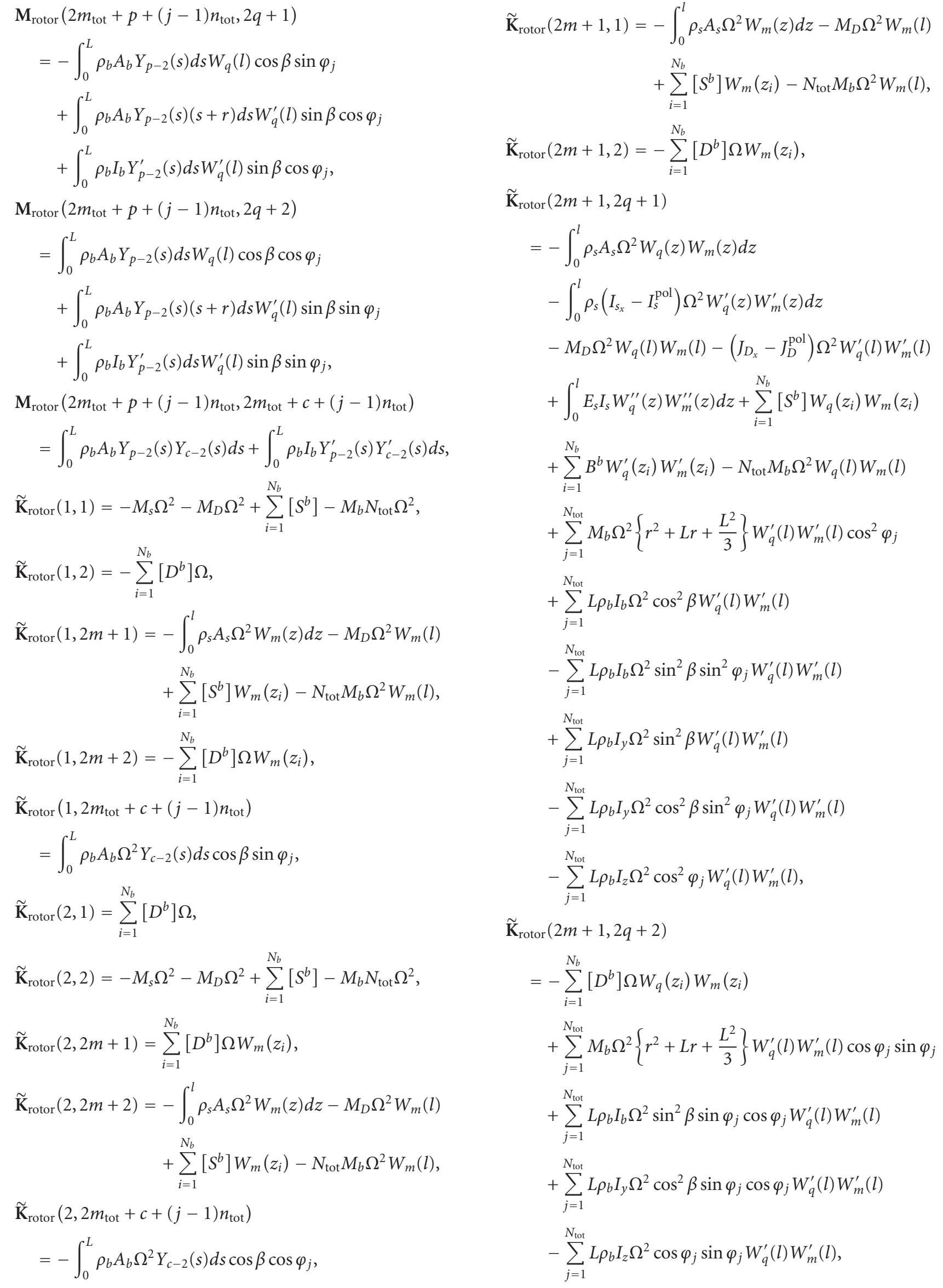

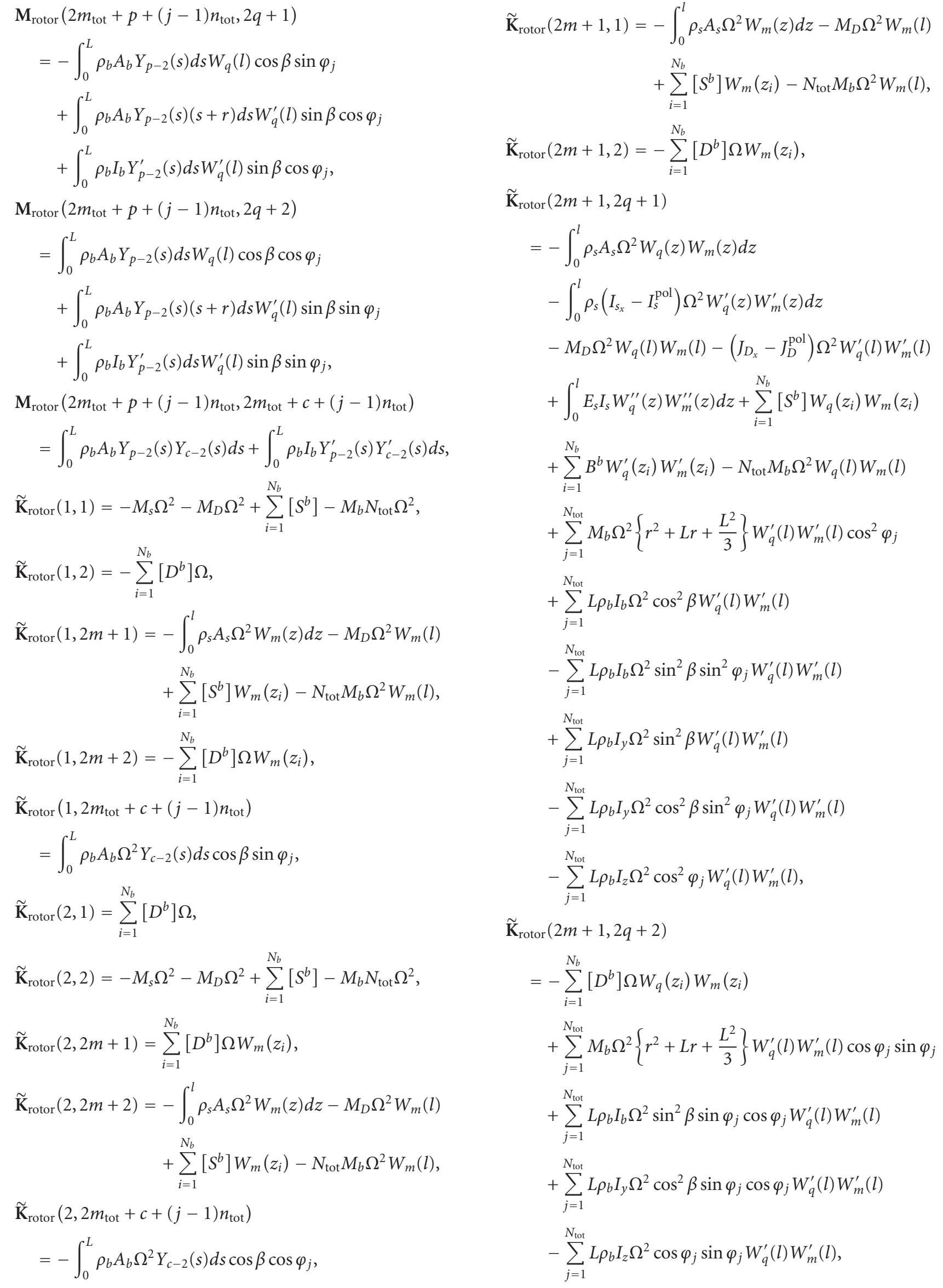

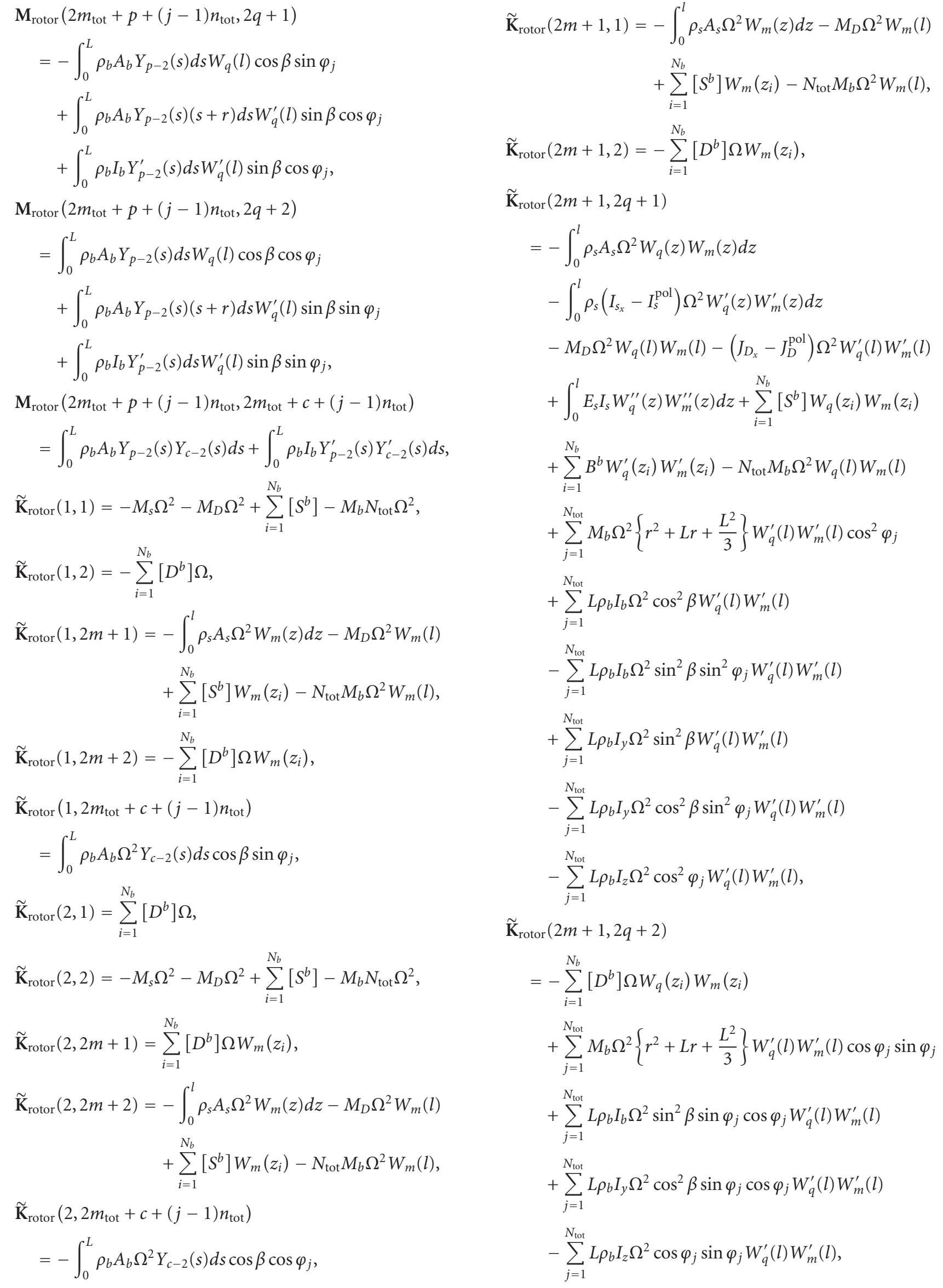

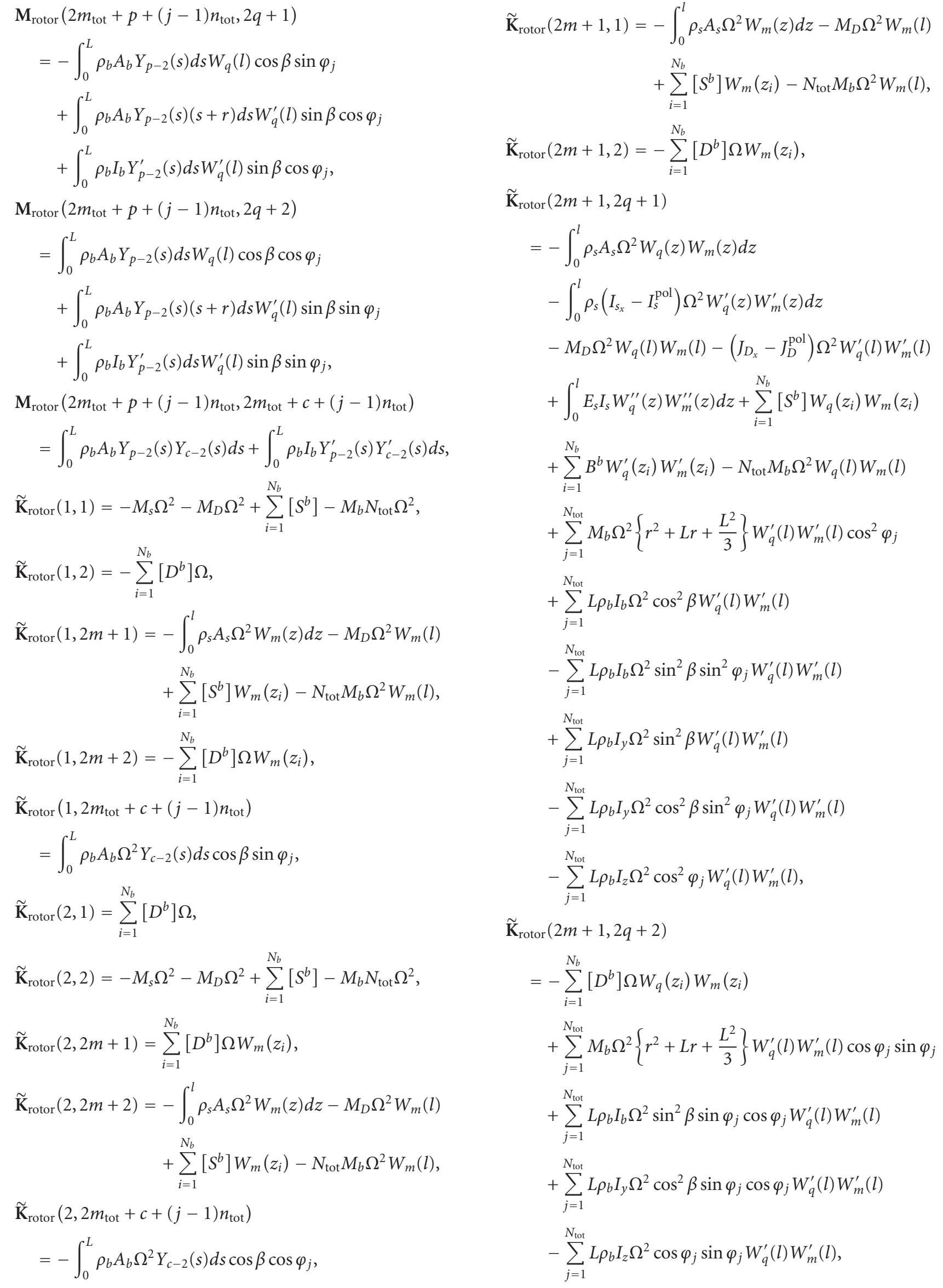

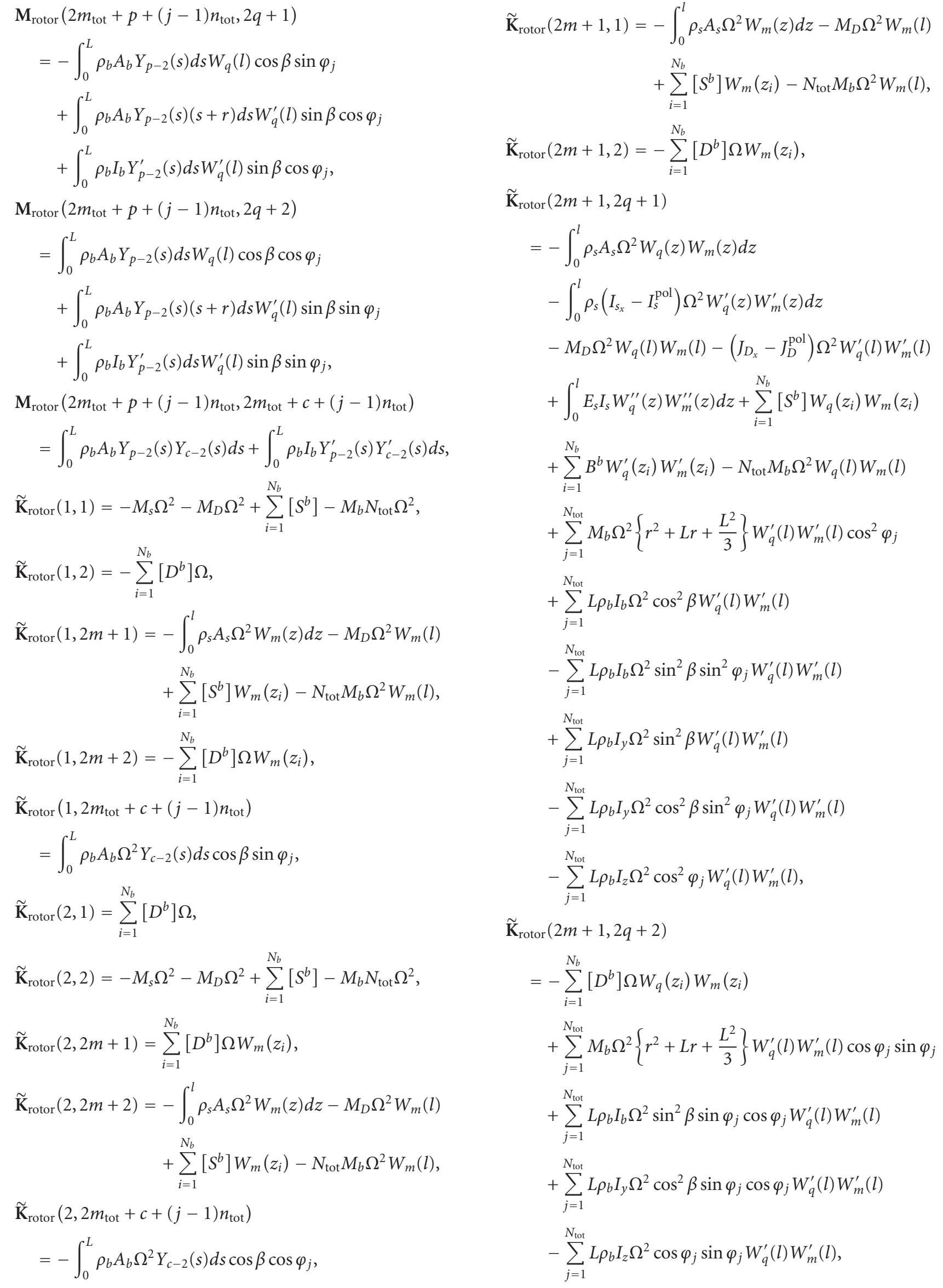

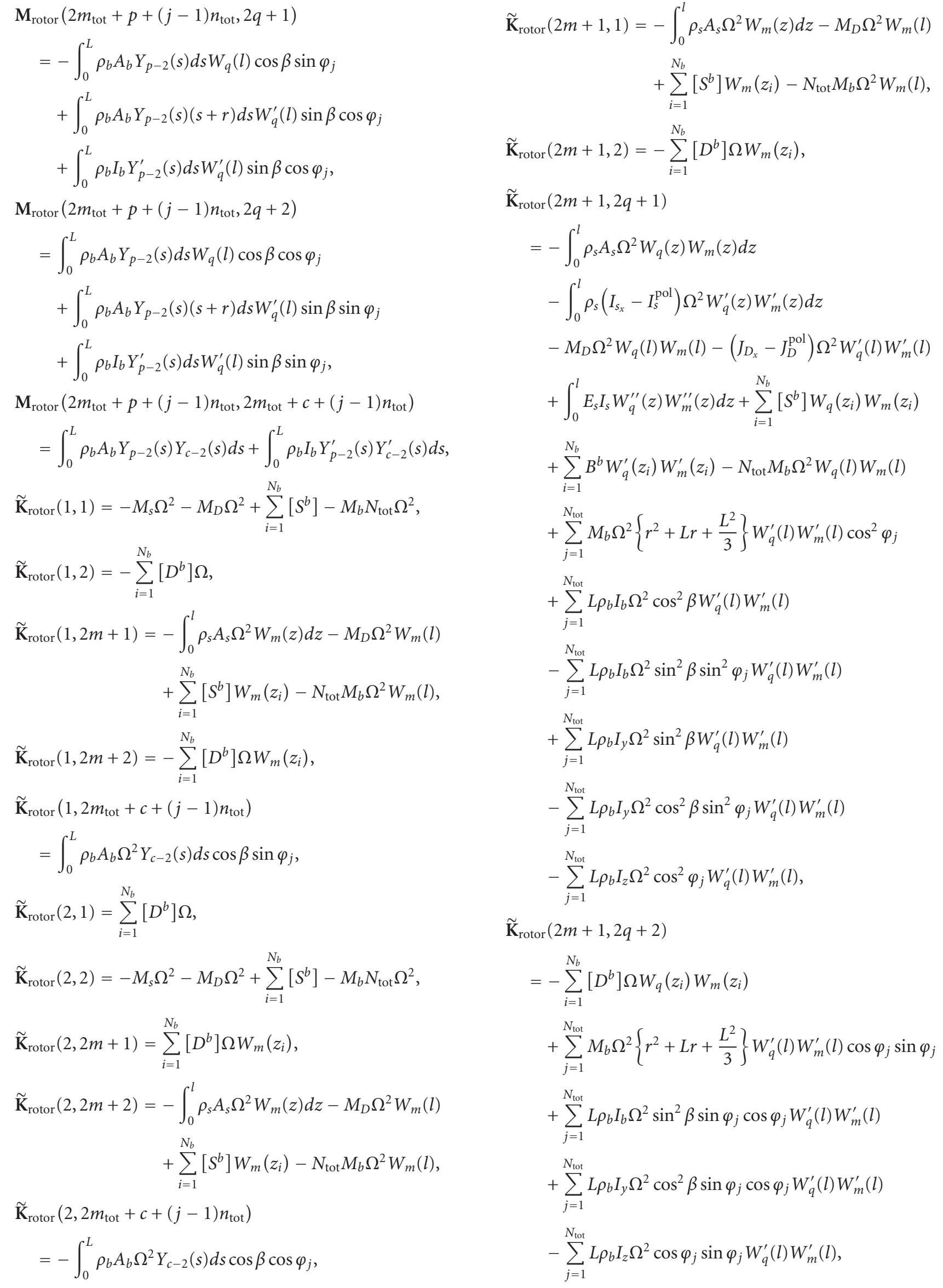

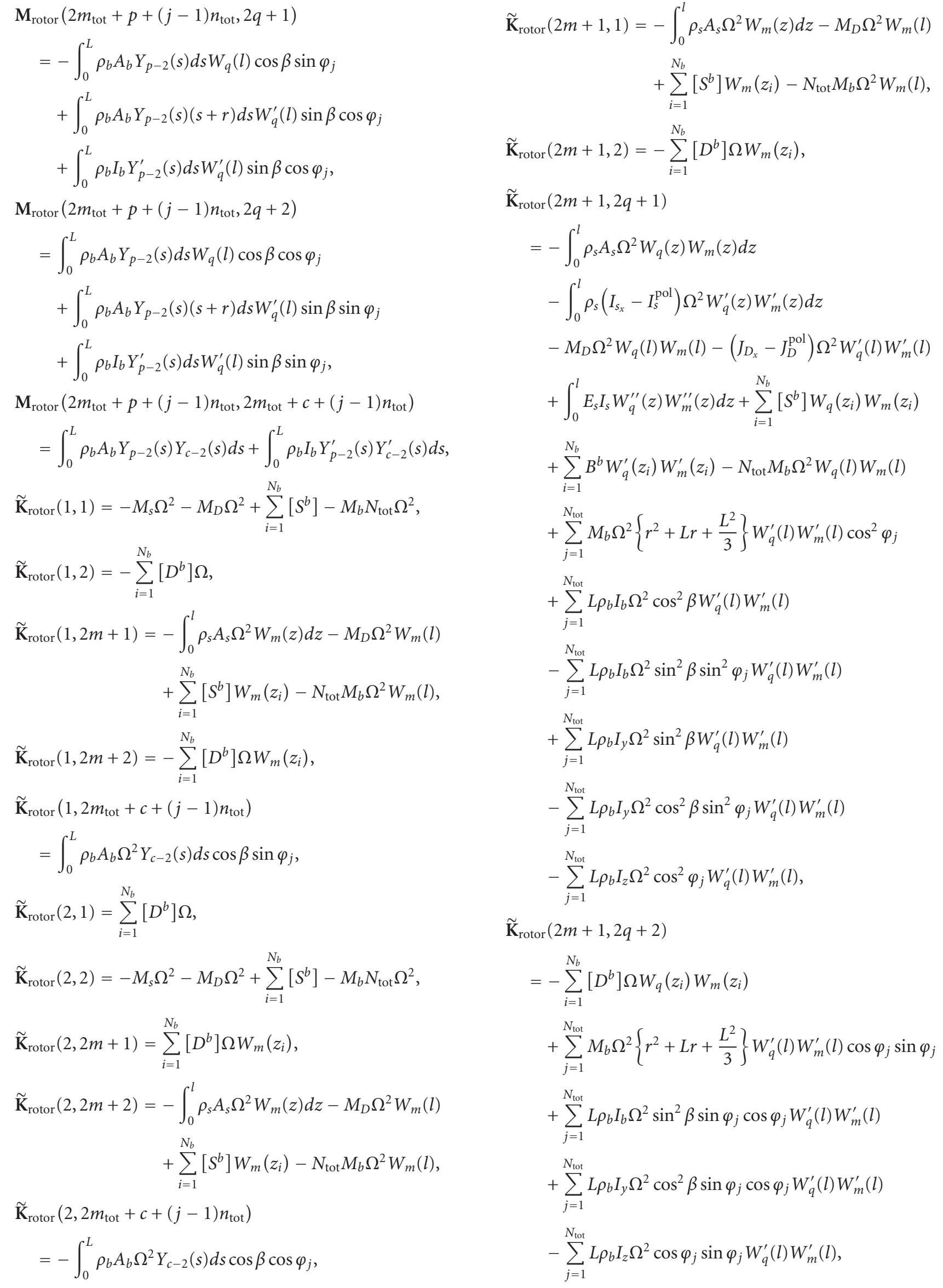

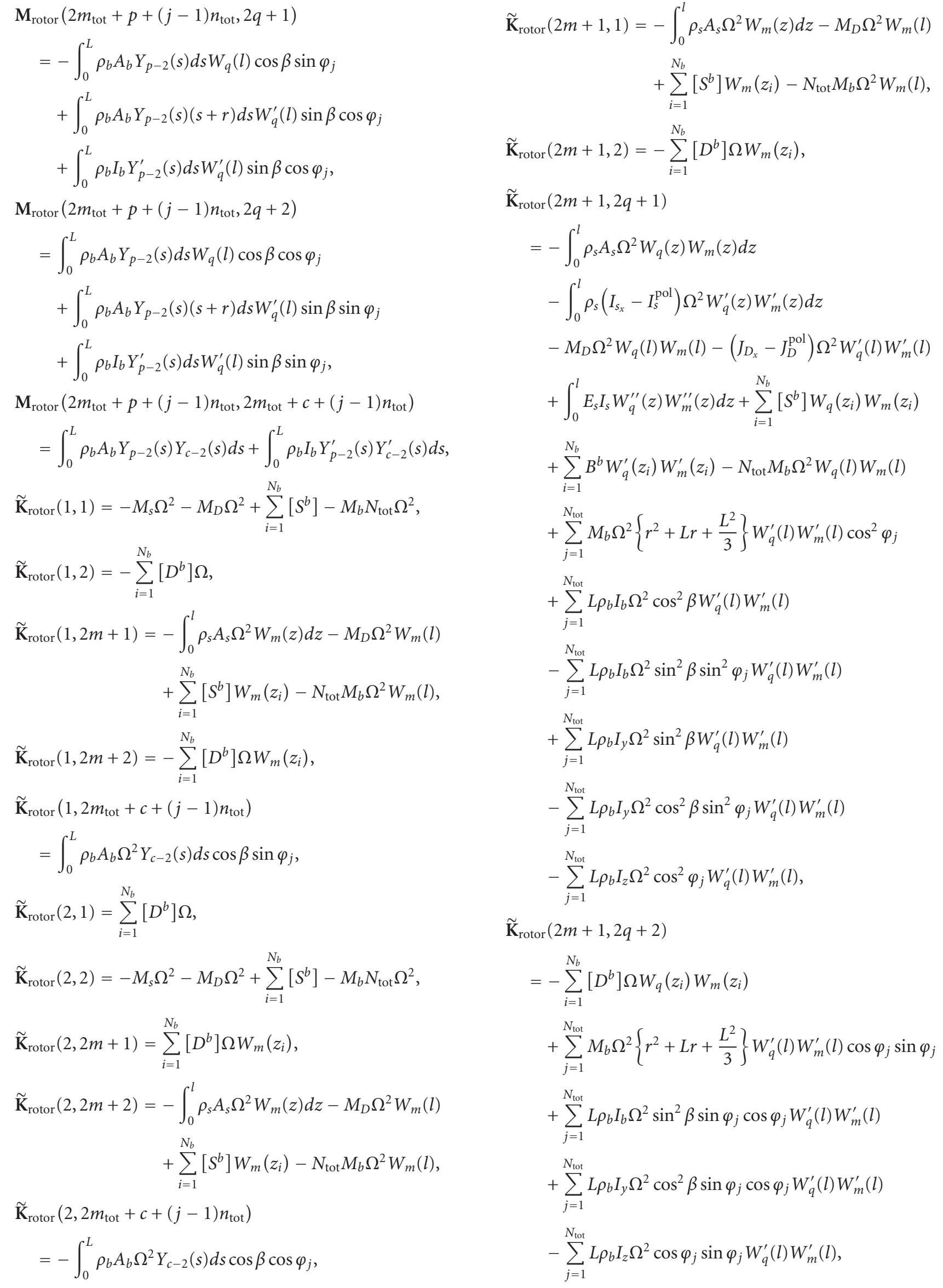

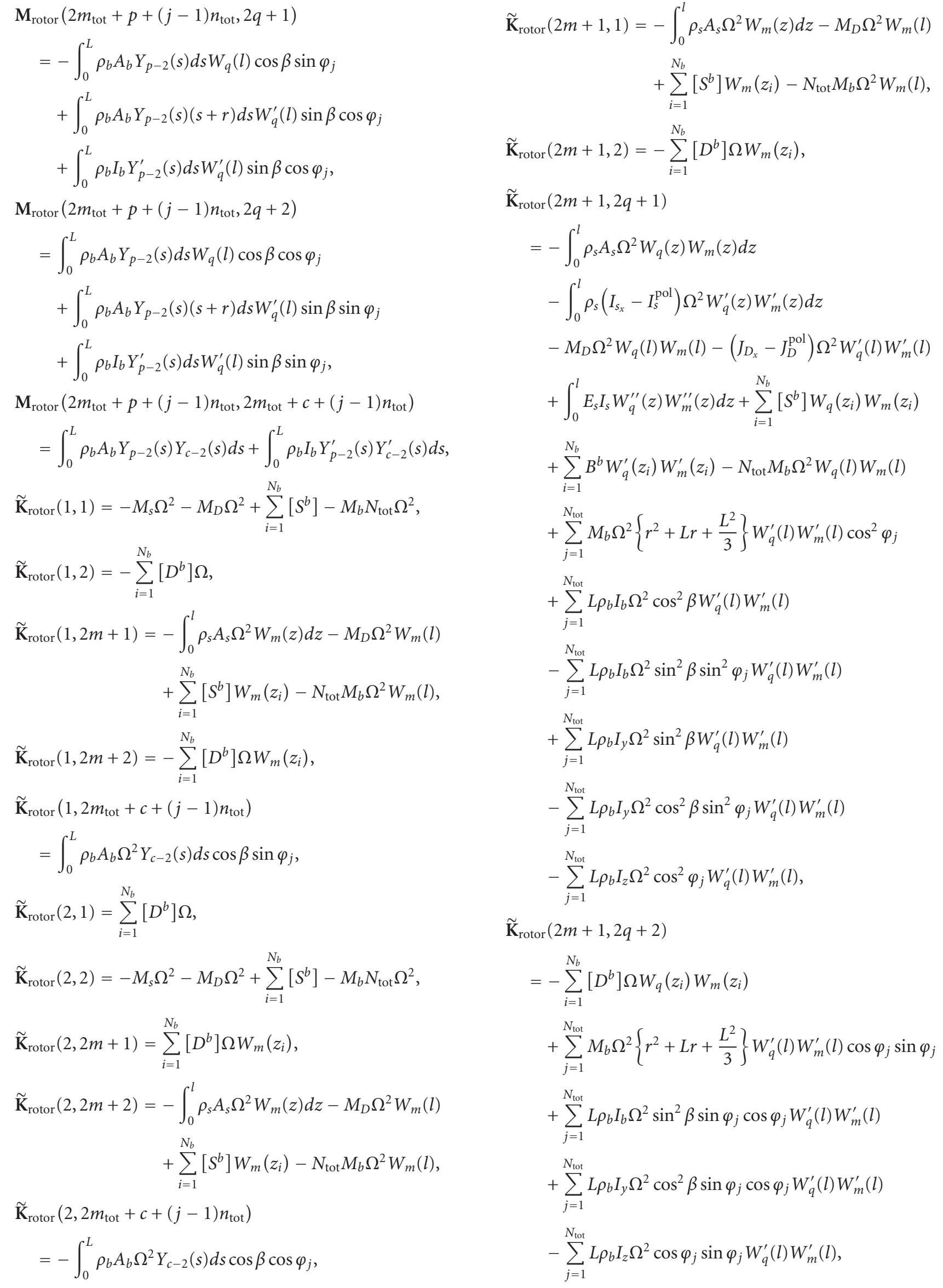

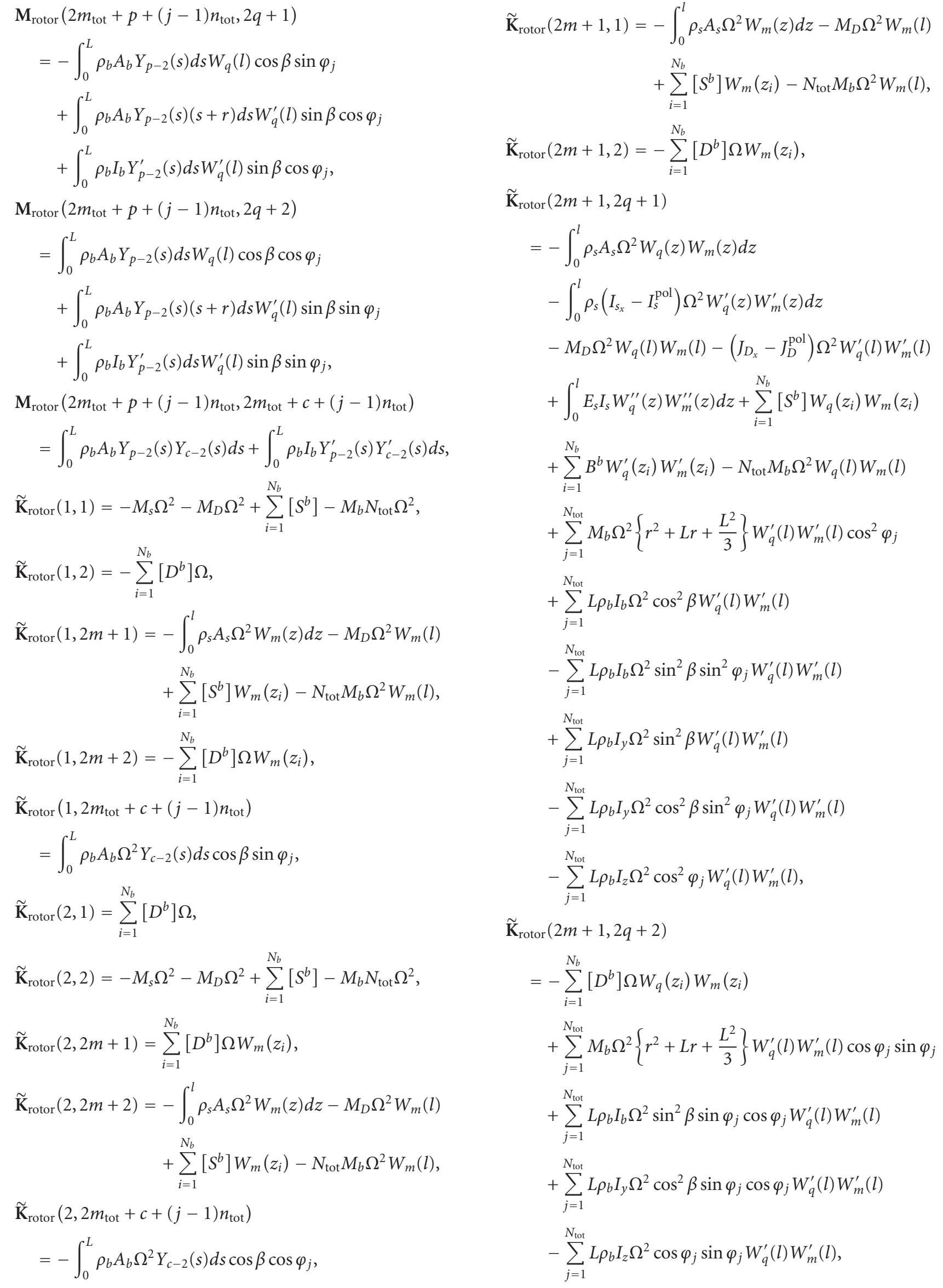

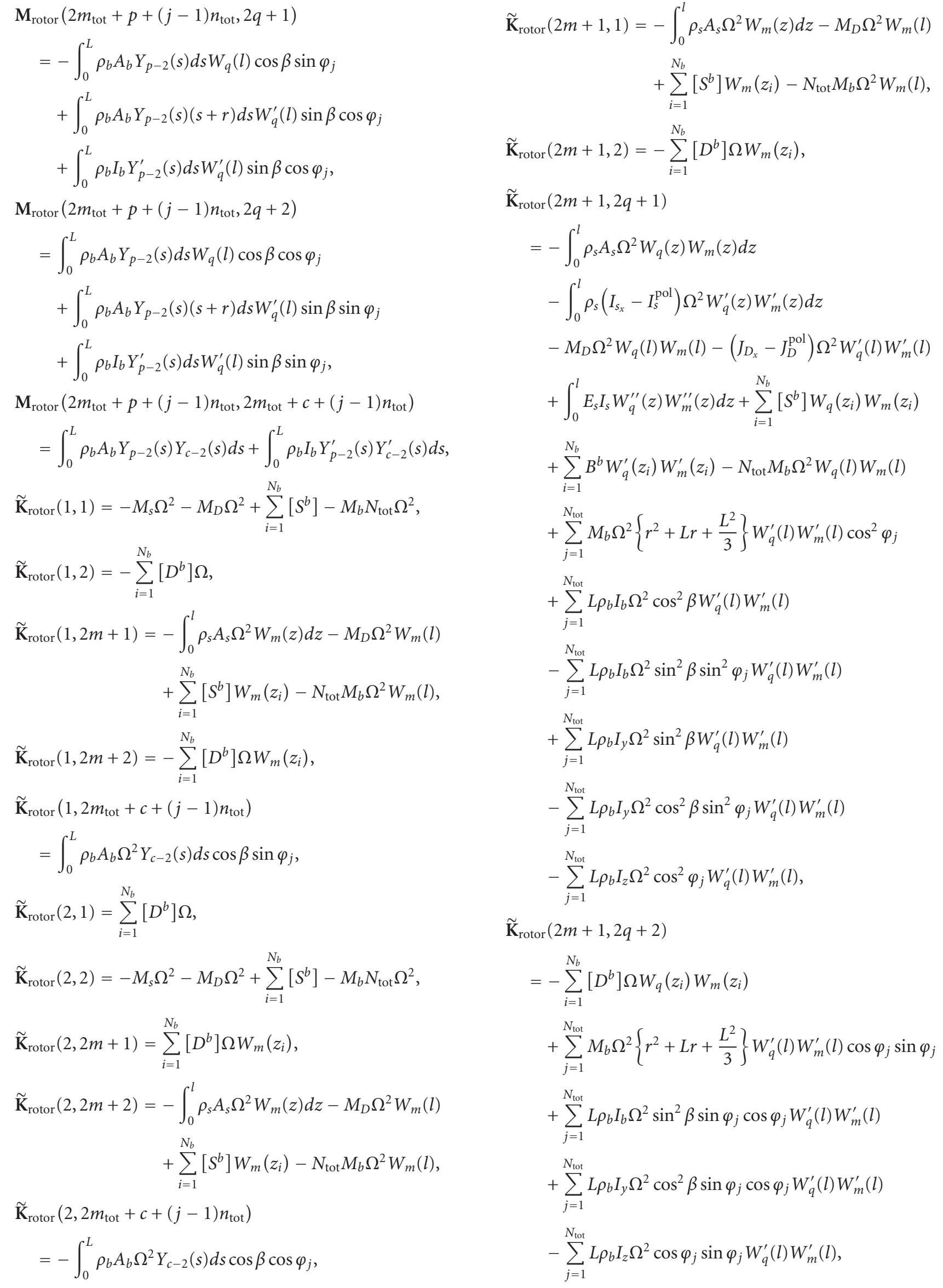

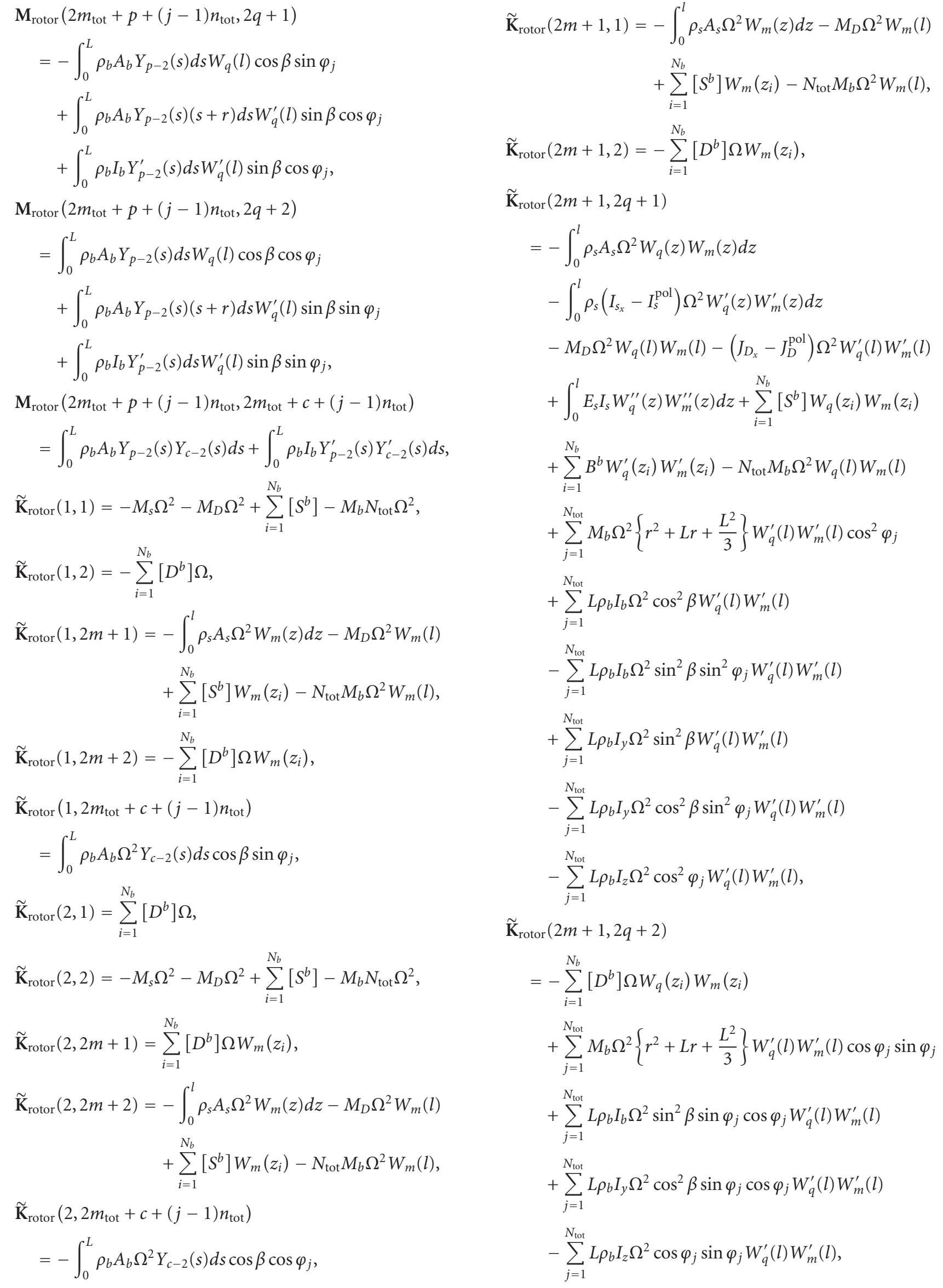

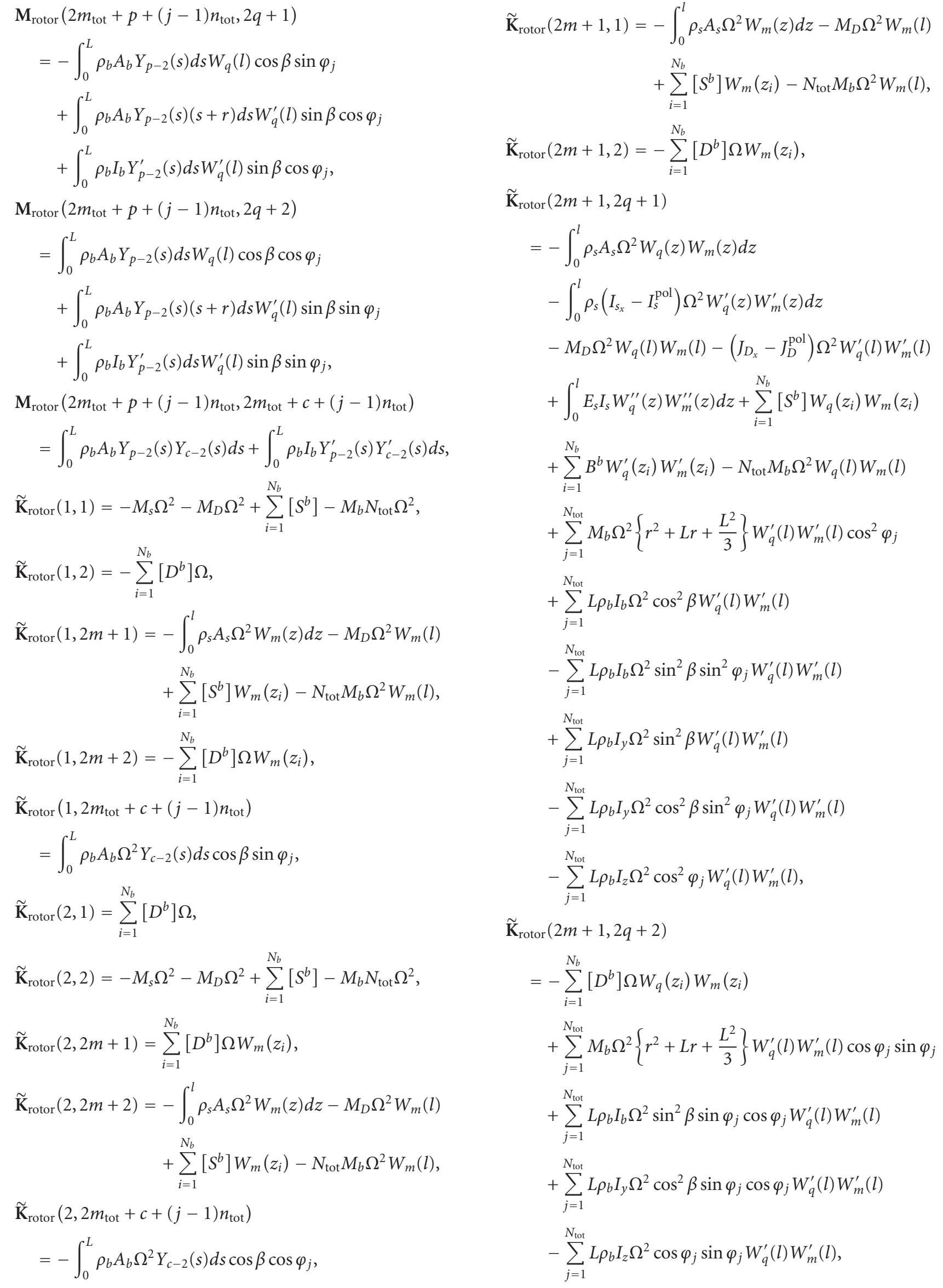


$\widetilde{\mathbf{K}}_{\text {rotor }}\left(2 m+1,2 m_{\text {tot }}+c+(j-1) n_{\text {tot }}\right)$

$=\int_{0}^{L} \rho_{b} A_{b} \Omega^{2} Y_{c-2}(s) d s W_{m}(l) \cos \beta \sin \varphi_{j}$

$+\int_{0}^{L} \rho_{b} A_{b} \Omega^{2} Y_{c-2}(s)(s+r) d s W_{m}^{\prime}(l) \sin \beta \cos \varphi_{j}$

$+\int_{0}^{L} \rho_{b} I_{y} \Omega^{2} Y_{c-2}^{\prime}(s) d s W_{m}^{\prime}(l) \sin \beta \cos \varphi_{j}$

$-\int_{0}^{L} \rho_{b} I_{z} \Omega^{2} Y_{c-2}^{\prime}(s) d s W_{m}^{\prime}(l) \sin \beta \cos \varphi_{j}$,

$\widetilde{\mathbf{K}}_{\text {rotor }}(2 m+2,1)=\sum_{i=1}^{N_{b}}\left[D^{b}\right] \Omega W_{m}\left(z_{i}\right)$,

$\widetilde{\mathbf{K}}_{\text {rotor }}(2 m+2,2)$

$=-\int_{0}^{l} \rho_{s} A_{s} \Omega^{2} W_{m}(z) d z-M_{D} \Omega^{2} W_{m}(l)$

$+\sum_{i=1}^{N_{b}}\left[S^{b}\right] W_{m}\left(z_{i}\right)-N_{\mathrm{tot}} M_{b} \Omega^{2} W_{m}(l)$

$\widetilde{\mathbf{K}}_{\text {rotor }}(2 m+2,2 q+1)$

$=\sum_{i=1}^{N_{b}}\left[D^{b}\right] \Omega W_{q}\left(z_{i}\right) W_{m}\left(z_{i}\right)$

$+\sum_{j=1}^{N_{\text {tot }}} M_{b} \Omega^{2}\left\{r^{2}+L r+\frac{L^{2}}{3}\right\} W_{q}^{\prime}(l) W_{m}^{\prime}(l) \cos \varphi_{j} \sin \varphi_{j}$

$+\sum_{j=1}^{N_{\text {tot }}} L \rho_{b} I_{b} \Omega^{2} \sin ^{2} \beta \sin \varphi_{j} \cos \varphi_{j} W_{q}^{\prime}(l) W_{m}^{\prime}(l)$

$+\sum_{j=1}^{N_{\text {tot }}} L \rho_{b} I_{y} \Omega^{2} \cos ^{2} \beta \sin \varphi_{j} \cos \varphi_{j} W_{q}^{\prime}(l) W_{m}^{\prime}(l)$

$-\sum_{j=1}^{N_{\text {tot }}} L \rho_{b} I_{z} \Omega^{2} \cos \varphi_{j} \sin \varphi_{j} W_{q}^{\prime}(l) W_{m}^{\prime}(l)$,

$\widetilde{\mathbf{K}}_{\text {rotor }}(2 m+2,2 q+2)$

$$
\begin{aligned}
= & -\int_{0}^{l} \rho_{s} A_{s} \Omega^{2} W_{q}(z) W_{m}(z) d z \\
& -\int_{0}^{l} \rho_{s}\left(I_{s_{y}}-I_{s}^{\mathrm{pol}}\right) \Omega^{2} W_{q}^{\prime}(z) W_{m}^{\prime}(z) d z \\
& -M_{D} \Omega^{2} W_{q}(l) W_{m}(l)-\left(J_{D_{y}}-J_{D}^{\mathrm{pol}}\right) \Omega^{2} W_{q}^{\prime}(l) W_{m}^{\prime}(l) \\
& +\int_{0}^{l} E_{s} I_{s} W_{q}^{\prime \prime}(z) W_{m}^{\prime \prime}(z) d z+\sum_{i=1}^{N_{b}}\left[S^{b}\right] W_{q}\left(z_{i}\right) W_{m}\left(z_{i}\right) \\
& +\sum_{i=1}^{N_{b}} B^{b} W_{q}^{\prime}\left(z_{i}\right) W_{m}^{\prime}\left(z_{i}\right)-N_{\mathrm{tot}} M_{b} \Omega^{2} W_{q}(l) W_{m}(l) \\
& +\sum_{j=1}^{N_{\mathrm{tot}}} M_{b} \Omega^{2}\left\{r^{2}+L r+\frac{L^{2}}{3}\right\} W_{q}^{\prime}(l) W_{m}^{\prime}(l) \sin ^{2} \varphi_{j} \\
& +\sum_{j=1}^{N_{\mathrm{tot}}} L \rho_{b} I_{b} \Omega^{2} \cos ^{2} \beta W_{q}^{\prime}(l) W_{m}^{\prime}(l)
\end{aligned}
$$

$-\sum_{j=1}^{N_{\mathrm{tot}}} L \rho_{b} I_{b} \Omega^{2} \sin ^{2} \beta \cos ^{2} \varphi_{j} W_{q}^{\prime}(l) W_{m}^{\prime}(l)$

$+\sum_{j=1}^{N_{\text {tot }}} L \rho_{b} I_{y} \Omega^{2} \sin ^{2} \beta W_{q}^{\prime}(l) W_{m}^{\prime}(l)$

$-\sum_{j=1}^{N_{\text {tot }}} L \rho_{b} I_{y} \Omega^{2} \cos ^{2} \beta \cos ^{2} \varphi_{j} W_{q}^{\prime}(l) W_{m}^{\prime}(l)$

$-\sum_{j=1}^{N_{\text {tot }}} L \rho_{b} I_{z} \Omega^{2} \sin ^{2} \varphi_{j} W_{q}^{\prime}(l) W_{m}^{\prime}(l)$,

$\widetilde{\mathbf{K}}_{\text {rotor }}\left(2 m+2,2 m_{\text {tot }}+c+(j-1) n_{\text {tot }}\right)$

$=-\int_{0}^{L} \rho_{b} A_{b} \Omega^{2} Y_{c-2}(s) d s W_{m}(l) \cos \beta \cos \varphi_{j}$

$+\int_{0}^{L} \rho_{b} A_{b} \Omega^{2} Y_{c-2}(s)(s+r) d s W_{m}^{\prime}(l) \sin \beta \sin \varphi_{j}$

$+\int_{0}^{L} \rho_{b} I_{y} \Omega^{2} Y_{c-2}^{\prime}(s) d s W_{m}^{\prime}(l) \sin \beta \sin \varphi_{j}$

$-\int_{0}^{L} \rho_{b} I_{z} \Omega^{2} Y_{c-2}^{\prime}(s) d s W_{m}^{\prime}(l) \sin \beta \sin \varphi_{j}$,

$\widetilde{\mathbf{K}}_{\text {rotor }}\left(2 m_{\text {tot }}+c+(j-1) n_{\text {tot }}, 1\right)$

$=\int_{0}^{L} \rho_{b} A_{b} \Omega^{2} Y_{p-2}(s) d s \cos \beta \sin \varphi_{j}$,

$\widetilde{\mathbf{K}}_{\text {rotor }}\left(2 m_{\text {tot }}+c+(j-1) n_{\text {tot }}, 2\right)$

$=-\int_{0}^{L} \rho_{b} A_{b} \Omega^{2} Y_{p-2}(s) d s \cos \beta \cos \varphi_{j}$,

$\widetilde{\mathbf{K}}_{\text {rotor }}\left(2 m_{\text {tot }}+c+(j-1) n_{\text {tot }}, 2 q+1\right)$

$=\int_{0}^{L} \rho_{b} A_{b} \Omega^{2} Y_{p-2}(s) d s W_{q}(l) \cos \beta \sin \varphi_{j}$

$+\int_{0}^{L} \rho_{b} A_{b} \Omega^{2} Y_{p-2}(s)(s+r) d s W_{q}^{\prime}(l) \sin \beta \cos \varphi_{j}$

$+\int_{0}^{L} \rho_{b} I_{y} \Omega^{2} Y_{p-2}^{\prime}(s) d s W_{q}^{\prime}(l) \sin \beta \cos \varphi_{j}$

$-\int_{0}^{L} \rho_{b} I_{z} \Omega^{2} Y_{p-2}^{\prime}(s) d s W_{q}^{\prime}(l) \sin \beta \cos \varphi_{j}$,

$\widetilde{\mathbf{K}}_{\text {rotor }}\left(2 m_{\text {tot }}+c+(j-1) n_{\text {tot }}, 2 q+2\right)$

$=-\int_{0}^{L} \rho_{b} A_{b} \Omega^{2} Y_{p-2}(s) d s W_{q}(l) \cos \beta \cos \varphi_{j}$

$+\int_{0}^{L} \rho_{b} A_{b} \Omega^{2} Y_{p-2}(s)(s+r) d s W_{q}^{\prime}(l) \sin \beta \sin \varphi_{j}$

$+\int_{0}^{L} \rho_{b} I_{y} \Omega^{2} Y_{p-2}^{\prime}(s) d s W_{q}^{\prime}(l) \sin \beta \sin \varphi_{j}$

$-\int_{0}^{L} \rho_{b} I_{z} \Omega^{2} Y_{p-2}^{\prime}(s) d s W_{q}^{\prime}(l) \sin \beta \sin \varphi_{j}$,

$\widetilde{\mathbf{K}}_{\text {rotor }}\left(2 m_{\mathrm{tot}}+p+(j-1) n_{\mathrm{tot}}, 2 m_{\mathrm{tot}}+c+(j-1) n_{\mathrm{tot}}\right)$

$=-\int_{0}^{L} \rho_{b} A_{b} \Omega^{2} Y_{p-2}(s) Y_{c-2}(s) d s \cos ^{2} \beta$ 


$$
\begin{aligned}
& +\int_{0}^{L} E_{b} I_{b} Y_{p-2}^{\prime \prime}(s) Y_{c-2}^{\prime \prime}(s) d s \\
& +\int_{0}^{L} \rho_{b} A_{b} \Omega^{2} \frac{R^{2}-(s+r)^{2}}{2} Y_{p-2}^{\prime}(s) Y_{c-2}^{\prime}(s) d s \\
& +\int_{0}^{L} \rho_{b} I_{y} \Omega^{2} Y_{c-2}^{\prime}(s) Y_{p-2}^{\prime}(s) d s \sin ^{2} \beta \\
& -\int_{0}^{L} \rho_{b} I_{z} \Omega^{2} Y_{c-2}^{\prime}(s) Y_{p-2}^{\prime}(s) d s \sin ^{2} \beta, \\
& \widetilde{\mathbf{C}}_{\text {rotor }}(1,1)=\sum_{i=1}^{N_{b}}\left[D^{b}\right] \\
& \widetilde{\mathbf{C}}_{\text {rotor }}(1,2)=-2 M_{s} \Omega-2 M_{D} \Omega-2 M_{b} N_{\text {tot }} \Omega \text {, } \\
& \widetilde{\mathbf{C}}_{\text {rotor }}(1,2 m+1)=\sum_{i=1}^{N_{b}}\left[D^{b}\right] W_{m}\left(z_{i}\right), \\
& \widetilde{\mathbf{C}}_{\text {rotor }}(1,2 m+2) \\
& =-2 \int_{0}^{l} \rho_{s} A_{s} \Omega W_{m}(z) d z-2 M_{D} \Omega W_{m}(l) \\
& -2 N_{\text {tot }} M_{b} \Omega W_{m}(l), \\
& \widetilde{\mathbf{C}}_{\text {rotor }}\left(1,2 m_{\text {tot }}+c+(j-1) n_{\text {tot }}\right) \\
& =-2 \int_{0}^{L} \rho_{b} A_{b} \Omega Y_{c-2}(s) d s \cos \beta \cos \varphi_{j}, \\
& \widetilde{\mathbf{C}}_{\text {rotor }}(2,1)=2 M_{s} \Omega+2 M_{D} \Omega+2 M_{b} N_{\text {tot }} \Omega \text {, } \\
& \widetilde{\mathbf{C}}_{\text {rotor }}(2,2)=\sum_{i=1}^{N_{b}}\left[D^{b}\right] \text {, } \\
& \widetilde{\mathbf{C}}_{\text {rotor }}(2,2 m+1) \\
& =2 \int_{0}^{l} \rho_{s} A_{s} \Omega W_{m}(z) d z+2 M_{D} \Omega W_{m}(l) \\
& +2 N_{\text {tot }} M_{b} \Omega W_{m}(l), \\
& \widetilde{\mathbf{C}}_{\text {rotor }}(2,2 m+2)=\sum_{i=1}^{N_{b}}\left[D^{b}\right] W_{m}\left(z_{i}\right) \text {, } \\
& \widetilde{\mathbf{C}}_{\text {rotor }}\left(2,2 m_{\text {tot }}+c+(j-1) n_{\text {tot }}\right) \\
& =-2 \int_{0}^{L} \rho_{b} A_{b} \Omega Y_{c-2}(s) d s \cos \beta \sin \varphi_{j}, \\
& \widetilde{\mathbf{C}}_{\text {rotor }}(2 m+1,1)=\sum_{i=1}^{N_{b}}\left[D^{b}\right] W_{m}\left(z_{i}\right) \text {, } \\
& \widetilde{\mathbf{C}}_{\text {rotor }}(2 m+1,2) \\
& =-2 \int_{0}^{l} \rho_{s} A_{s} \Omega W_{m}(z) d z-2 M_{D} \Omega W_{m}(l) \\
& -2 N_{\text {tot }} M_{b} \Omega W_{m}(l), \\
& \widetilde{\mathbf{C}}_{\text {rotor }}(2 m+1,2 q+1) \\
& =\sum_{i=1}^{N_{b}}\left[D^{b}\right] W_{q}\left(z_{i}\right) W_{m}\left(z_{i}\right)+\int_{0}^{l} \eta_{s} E_{s} I_{s} \Omega W_{q}^{\prime \prime}(z) W_{m}^{\prime \prime}(z) d z, \\
& \widetilde{\mathbf{C}}_{\text {rotor }}(2 m+1,2 q+2) \\
& =-2 \int_{0}^{l} \rho_{s} A_{s} \Omega W_{q}(z) W_{m}(z) d z \\
& -\int_{0}^{l} \rho_{s}\left(I_{s_{y}}+I_{s_{x}}\right) \Omega W_{q}^{\prime}(z) W_{m}^{\prime}(z) d z \\
& -2 M_{D} \Omega W_{q}(l) W_{m}(l)-2\left(J_{D_{y}}+J_{D_{x}}\right) \Omega W_{q}^{\prime}(l) W_{m}^{\prime}(l) \\
& +\int_{0}^{l} \rho_{s} I_{s}^{\mathrm{pol}} \Omega W_{q}^{\prime}(z) W_{m}^{\prime}(z) d z+J_{D}^{\mathrm{pol}} \Omega W_{q}^{\prime}(l) W_{m}^{\prime}(l) \\
& -2 N_{\text {tot }} M_{b} \Omega W_{q}(l) W_{m}(l) \\
& -\sum_{j=1}^{N_{\text {tot }}} L \rho_{b} I_{b} \Omega \sin ^{2} \beta W_{q}^{\prime}(l) W_{m}^{\prime}(l) \\
& +\sum_{j=1}^{N_{\text {tot }}} L \rho_{b} I_{b} \Omega \cos ^{2} \beta W_{q}^{\prime}(l) W_{m}^{\prime}(l) \\
& -\sum_{j=1}^{N_{\mathrm{tot}}} L \rho_{b} I_{y} \Omega \cos ^{2} \beta W_{q}^{\prime}(l) W_{m}^{\prime}(l) \\
& +\sum_{j=1}^{N_{\mathrm{tot}}} L \rho_{b} I_{y} \Omega \sin ^{2} \beta W_{q}^{\prime}(l) W_{m}^{\prime}(l) \\
& -\sum_{j=1}^{N_{\mathrm{tot}}} L \rho_{b} I_{z} \Omega W_{q}^{\prime}(l) W_{m}^{\prime}(l) \\
& \widetilde{\mathbf{C}}_{\text {rotor }}\left(2 m+1,2 m_{\text {tot }}+c+(j-1) n_{\text {tot }}\right) \\
& =-2 \int_{0}^{L} \rho_{b} A_{b} \Omega Y_{c-2}(s) d s W_{m}(l) \cos \beta \cos \varphi_{j} \\
& -2 \int_{0}^{L} \rho_{b} I_{b} \Omega Y_{c-2}^{\prime}(s) d s W_{m}^{\prime}(l) \sin \beta \sin \varphi_{j}, \\
& \widetilde{\mathbf{C}}_{\text {rotor }}(2 m+2,1) \\
& =2 \int_{0}^{l} \rho_{s} A_{s} \Omega W_{m}(z) d z+2 M_{D} \Omega W_{m}(l) \\
& +2 N_{\text {tot }} M_{b} \Omega W_{m}(l), \\
& \widetilde{\mathbf{C}}_{\text {rotor }}(2 m+2,2)=\sum_{i=1}^{N_{b}}\left[D^{b}\right] W_{m}\left(z_{i}\right), \\
& \widetilde{\mathbf{C}}_{\text {rotor }}(2 m+2,2 q+1) \\
& =2 \int_{0}^{l} \rho_{s} A_{s} \Omega W_{q}(z) W_{m}(z) d z \\
& +2 \int_{0}^{l} \rho_{s}\left(I_{s_{x}}+I_{s_{y}}\right) \Omega W_{q}^{\prime}(z) W_{m}^{\prime}(z) d z \\
& +2 M_{D} \Omega W_{q}(l) W_{m}(l) \\
& +2\left(J_{D_{x}}+J_{D_{y}}\right) \Omega W_{q}^{\prime}(l) W_{m}^{\prime}(l) \\
& -\int_{0}^{l} \rho_{s} I_{s}^{\mathrm{pol}} \Omega W_{q}^{\prime}(z) W_{m}^{\prime}(z) d z-J_{D}^{\mathrm{pol}} \Omega W_{q}^{\prime}(l) W_{m}^{\prime}(l)
\end{aligned}
$$




$$
\begin{aligned}
& +2 N_{\text {tot }} M_{b} \Omega W_{q}(l) W_{m}(l) \\
& +\sum_{j=1}^{N_{\text {tot }}} L \rho_{b} I_{b} \Omega \sin ^{2} \beta W_{q}^{\prime}(l) W_{m}^{\prime}(l) \\
& -\sum_{j=1}^{N_{\mathrm{tot}}} L \rho_{b} I_{b} \Omega \cos ^{2} \beta W_{q}^{\prime}(l) W_{m}^{\prime}(l) \\
& +\sum_{j=1}^{N_{\text {tot }}} L \rho_{b} I_{y} \Omega \cos ^{2} \beta W_{q}^{\prime}(l) W_{m}^{\prime}(l) \\
& -\sum_{j=1}^{N_{\text {tot }}} L \rho_{b} I_{y} \Omega \sin ^{2} \beta W_{q}^{\prime}(l) W_{m}^{\prime}(l) \\
& +\sum_{j=1}^{N_{\text {tot }}} L \rho_{b} I_{z} \Omega W_{q}^{\prime}(l) W_{m}^{\prime}(l) \\
& \widetilde{\mathbf{C}}_{\text {rotor }}(2 m+2,2 q+2) \\
& =\sum_{i=1}^{N_{b}}\left[D^{b}\right] W_{q}\left(z_{i}\right) W_{m}\left(z_{i}\right)+\int_{0}^{l} \eta_{s} E_{s} I_{s} \Omega W_{q}^{\prime \prime}(z) W_{m}^{\prime \prime}(z) d z, \\
& \widetilde{\mathbf{C}}_{\text {rotor }}\left(2 m+2,2 m_{\text {tot }}+c+(j-1) n_{\text {tot }}\right) \\
& =-2 \int_{0}^{L} \rho_{b} A_{b} \Omega Y_{c-2}(s) d s W_{m}(l) \cos \beta \sin \varphi_{j} \\
& +2 \int_{0}^{L} \rho_{b} I_{b} \Omega Y_{c-2}^{\prime}(s) d s W_{m}^{\prime}(l) \sin \beta \cos \varphi_{j}, \\
& \tilde{\mathbf{C}}_{\text {rotor }}\left(2 m_{\text {tot }}+c+(j-1) n_{\text {tot }}, 1\right) \\
& =2 \int_{0}^{L} \rho_{b} A_{b} \Omega Y_{p-2}(s) d s \cos \beta \cos \varphi_{j}, \\
& \tilde{\mathbf{C}}_{\text {rotor }}\left(2 m_{\text {tot }}+c+(j-1) n_{\text {tot }}, 2\right) \\
& =2 \int_{0}^{L} \rho_{b} A_{b} \Omega Y_{p-2}(s) d s \cos \beta \sin \varphi_{j}, \\
& \widetilde{\mathbf{C}}_{\text {rotor }}\left(2 m_{\text {tot }}+c+(j-1) n_{\text {tot }}, 2 m+1\right) \\
& =2 \int_{0}^{L} \rho_{b} A_{b} \Omega Y_{p-2}(s) d s W_{q}(l) \cos \beta \cos \varphi_{j} \\
& +2 \int_{0}^{L} \rho_{b} I_{b} \Omega Y_{p-2}^{\prime}(s) d s W_{q}^{\prime}(l) \sin \beta \sin \varphi_{j}, \\
& \widetilde{\mathbf{C}}_{\text {rotor }}\left(2 m_{\text {tot }}+c+(j-1) n_{\text {tot }}, 2 m+2\right) \\
& =2 \int_{0}^{L} \rho_{b} A_{b} \Omega Y_{p-2}(s) d s W_{q}(l) \cos \beta \sin \varphi_{j} \\
& -2 \int_{0}^{L} \rho_{b} I_{b} \Omega Y_{p-2}^{\prime}(s) d s W_{q}^{\prime}(l) \sin \beta \cos \varphi_{j}, \\
& \tilde{\mathbf{C}}\left(2 m_{\mathrm{tot}}+p+(j-1) n_{\mathrm{tot}}, 2 m_{\mathrm{tot}}+c+(j-1) n_{\mathrm{tot}}\right) \\
& =\int_{0}^{L} \eta_{b} E_{b} I_{b} Y_{p-2}^{\prime \prime}(s) Y_{c-2}^{\prime \prime}(s) d s .
\end{aligned}
$$

\section{NOMENCLATURE}

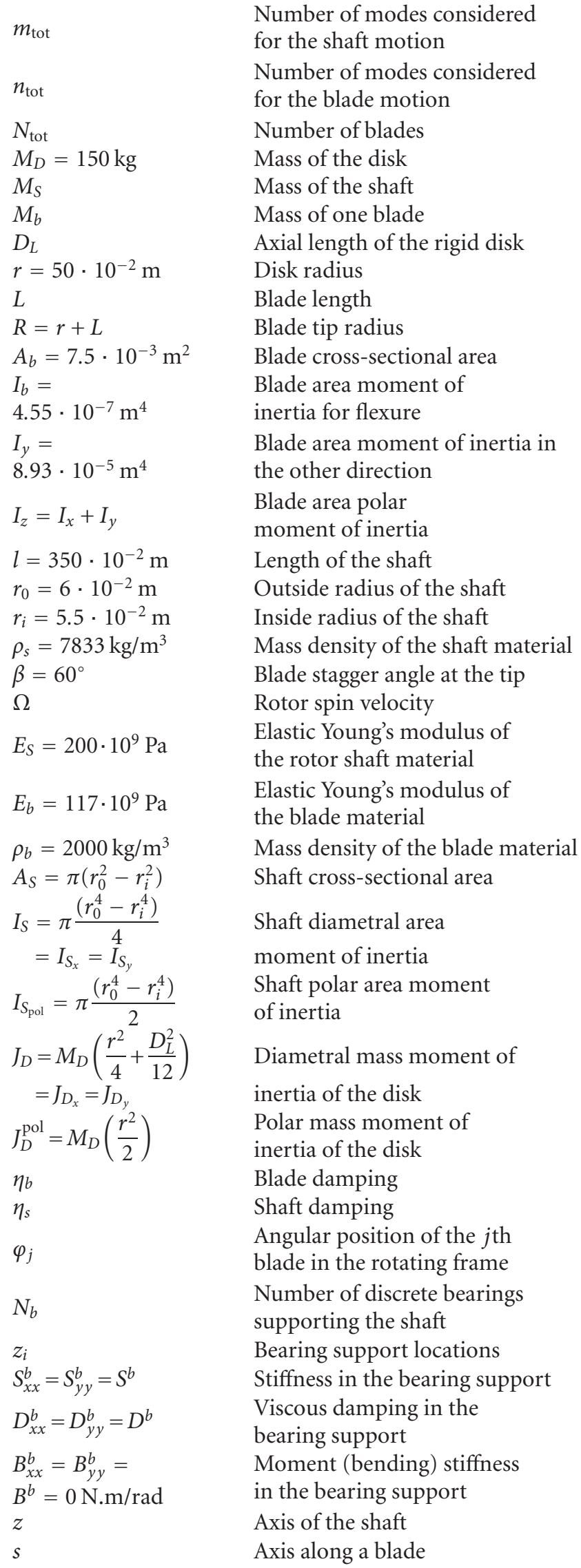




\section{REFERENCES}

[1] D. Childs, Turbomachinery Rotordynamics: Phenomena, Modeling, and Analysis, John Wiley \& Sons, New York, NY, USA, 1993.

[2] F. F. Ehrich, Handbook of Rotordynamics, McGraw-Hill, New York, NY, USA, 1992.

[3] T. Yamamoto and Y. Ishida, Linear and Nonlinear Rotordynamics: A Modern Treatment with Applications, John Wiley \& Sons, New York, NY, USA, 2001.

[4] S. H. Crandall, "Rotordynamics," in Nonlinear Dynamics and Stochastic Mechanics, W. Kliemann and N. S. Namachchivaya, Eds., pp. 1-44, CRC Press, Boca Raton, Fla, USA, 1995.

[5] S. H. Crandall and J. Dugundji, "Resonant whirling of aircraft propeller-engine systems," Journal of Applied Mechanics, vol. 48, pp. 929-935, 1981.

[6] G. Genta, "On the stability of rotating blade arrays," Journal of Sound and Vibration, vol. 273, no. 4-5, pp. 805-836, 2004.

[7] T. Gmür, Dynamique des structures: Analyse modale numérique, Presses Polytechniques et Universitaires Romandes, Lausanne, Switzerland, 1997.

[8] M. LaLanne and G. Ferraris, Rotordynamics Prediction in Engineering, John Wiley \& Sons, New York, NY, USA, 1990.

[9] S. K. Sinha, "Dynamic characteristics of a flexible bladed-rotor with Coulomb damping due to tip-rub," Journal of Sound and Vibration, vol. 273, no. 4-5, pp. 875-919, 2004.

[10] B. O. Al-Bedoor and A. A. Al-Qaisia, "Stability analysis of rotating blade bending vibration due to torsional excitation," Journal of Sound and Vibration, vol. 282, no. 3-5, pp. 10651083, 2005.

[11] Ö. Turhan and G. Bulut, "Dynamic stability of rotating blades (beams) eccentrically clamped to a shaft with fluctuating speed," Journal of Sound and Vibration, vol. 280, no. 3-5, pp. 945-964, 2005.

[12] A. W. Leissa, "On a curve veering aberration," Journal of Applied Mathematics and Physics, vol. 25, no. 1, pp. 99-111, 1974.

[13] C. Pierre, "Mode localization and eigenvalue loci veering phenomena in disordered structures," Journal of Sound and Vibration, vol. 126, no. 3, pp. 485-502, 1988. 

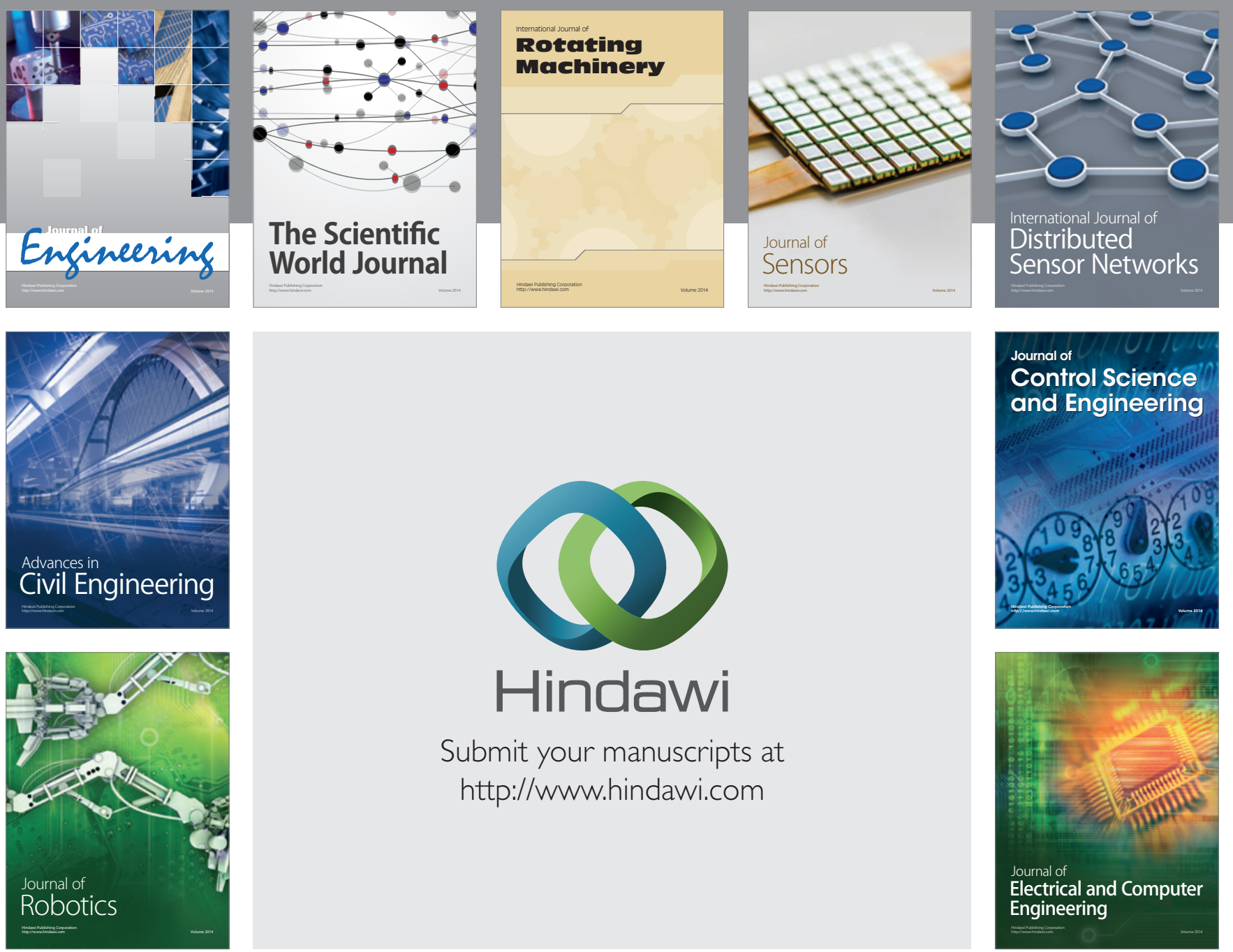

Submit your manuscripts at

http://www.hindawi.com
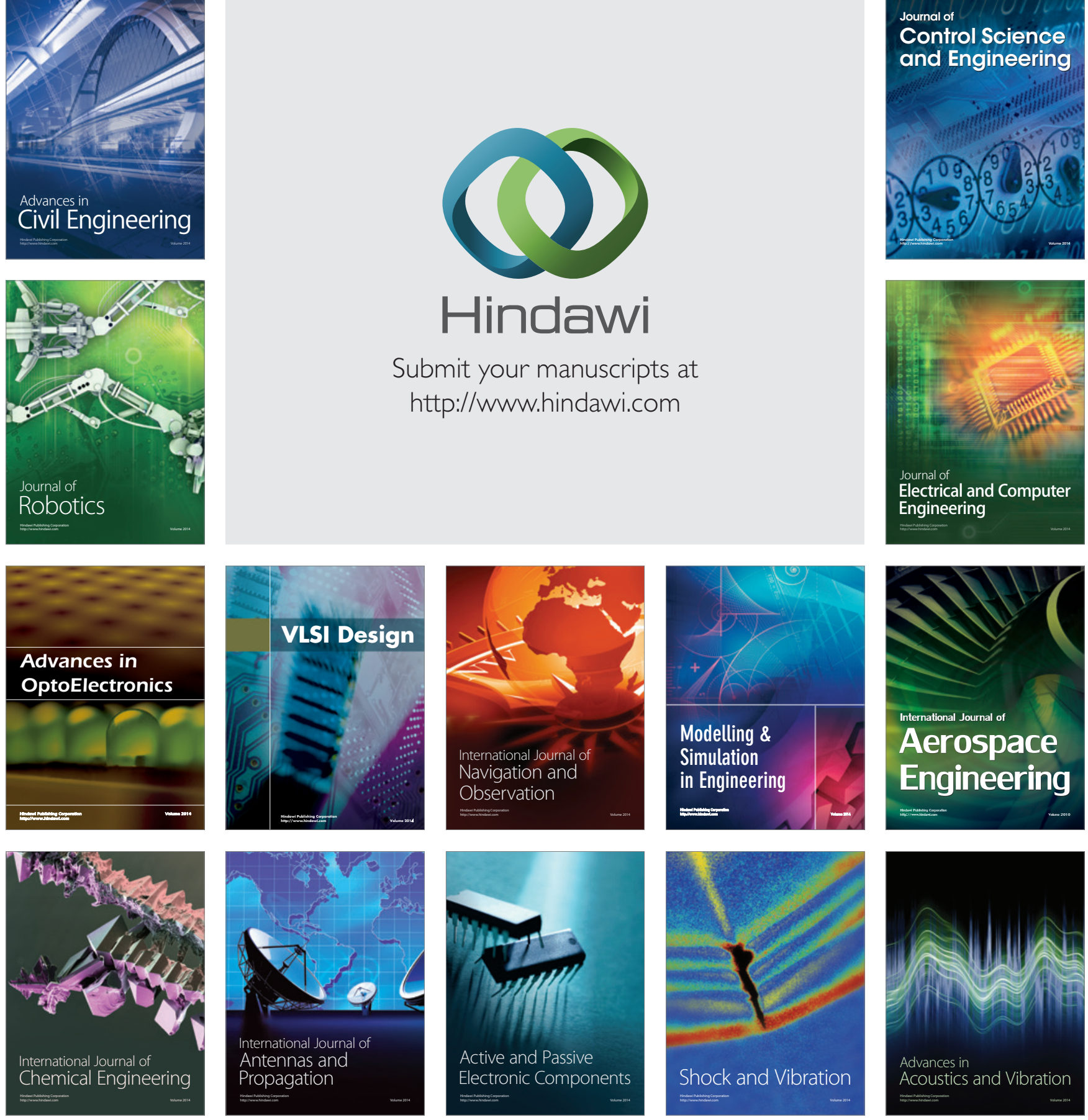